\title{
AJUSTE DE MODELOS MISTOS DE CLASSIFICAÇÃO DUPLA: PROCEDIMENTOS DO SISTEMA ESTATÍSTICO “SAS"
}

\author{
SÍLVIA HELENA VENTUROLI PERRI \\ Bacharel em Estatística
}

Orientador: Prof. Dr. ANTONIO FRANCISCO IEMMA

Tese apresentada à Escola Superior de Agricultura "Luiz de Queiroz", Universidade de São Paulo, para obtenção do título de Doutor em Agronomia, Área de Concentração: Estatística e Experimentação Agronômica.

P I R A C I C A B A

Estado de São Paulo - Brasil

Dezembro - 1998 
Dados Internacionais de Catalogação na Publicação (CIP)

DIVISĀO DE BIBLIOTECA E DOCUMENTAÇĀO - Campus "Luiz de Queiroz"/USP

Perri, Silvia Helena Venturoli

Ajuste de modelos mistos de classificação dupla: procedimentos do sistema estatistico "SAS" / Silvia Helena Venturoli Perri. - - Piracicaba, 1998.

$126 \mathrm{p}$.

Tese (doutorado) - - Escola Superior de Agricultura Luiz de Queiroz, 1998. Bibliografia.

1. Análise de varianncia 2. Estatística aplicada 3. Modelo matemático 4. Programa de computador 5. SAS I. Título 
A Deus,

pela vida e oportunidade concedida.

Aos meus pais,

pela perseverança.

Ao Fernando, Rodrigo e Tiago, pelo apoio, paciência e compreensão. 


\section{AGRADECIMENTOS}

Ao Professor Dr. Antonio Francisco Iemma por sua paciência, profissionalismo, confiança e orientação que contribuiu de forma decisiva para a realização deste trabalho.

Aos Professores do Departamento de Matemática e Estatística da ESALQ/USP, em especial ao Professor Dr. José Eduardo Corrente que auxiliou na redação do Summary.

Aos funcionários do Departamento de Matemática e Estatística da ESALQ/USP pela paciência e amizade.

Aos colegas do Curso de Pós-Graduação pela agradável convivência.

Aos colegas do Curso de Medicina Veterinária da UNESP/Araçatuba pelo apoio e compreensão.

À CAPES/PICDT pela bolsa de estudos que muito contribuiu para viabilizar o meu doutorado.

Ao Professor Dr. Orlando Saliba pelo incentivo para a realização deste trabalho.

Ao Fernando pelo apoio e incentivo sempre presente e aos meus filhos Rodrigo e Tiago pela paciência e horas de espera por uma atenção.

À minha família em especial à minha irmã pelo carinho, colaboração e estímulo.

A todos aqueles que de alguma forma contribuíram para a realização deste trabalho. 


\section{SUMÁRIO}

Página

RESUMO ..vi

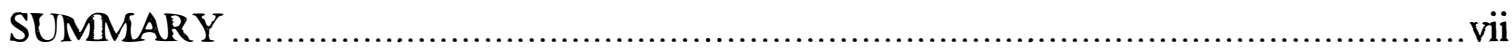

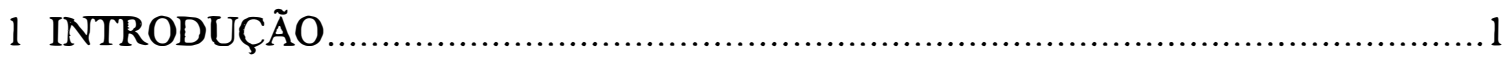

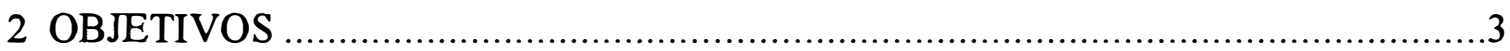

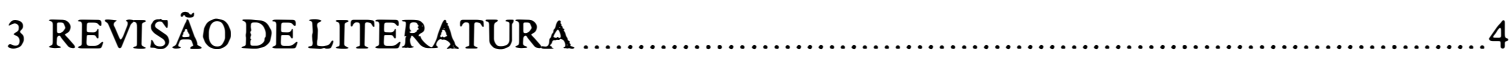

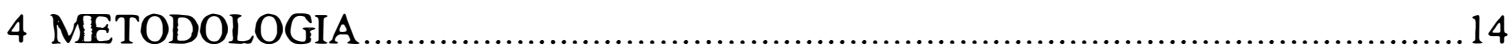

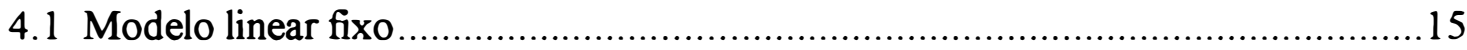

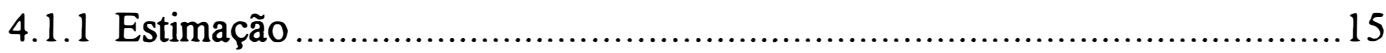

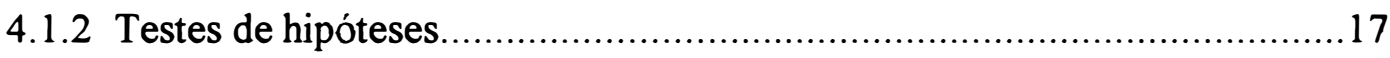

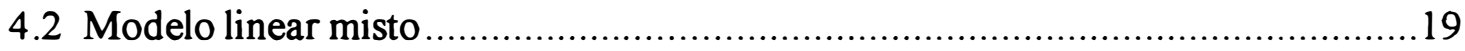

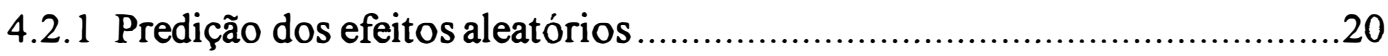

4.2.2 Estimação dos componentes de variância .............................................23

4.2.2.1 Método da análise de variância : ANOVA ..................................24

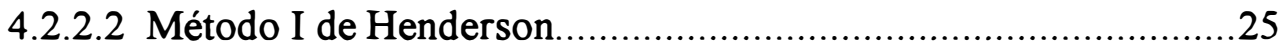

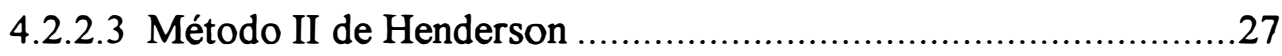

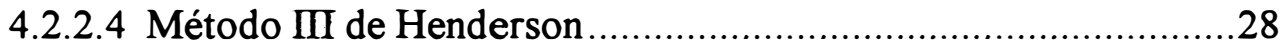

4.2.2.5 Método da máxima verossimilhança : ML ..................................

4.2.2.6 Método da máxima verossimilhança restrita : REML ..................33

4.2.2.7 Método de estimação quadrática não-viesada de norma mínima:

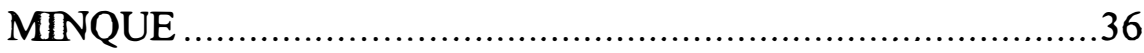

4.2.2.8 Método de estimação quadrática não-viesada de variância mínima :

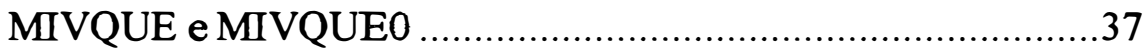

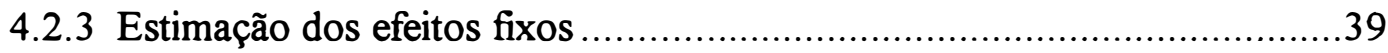


Página

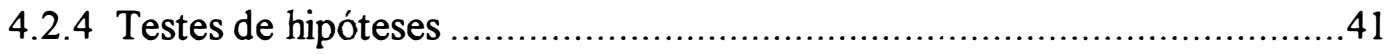

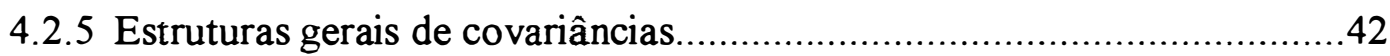

4.3 Ajuste de modelos mistos por meio do sistema estatístico SAS ........................46

4.3.1 Procedimento GLM : PROC GLM..................................................... 47

4.3.2 Procedimento VARCOMP : PROC VARCOMP .................................52

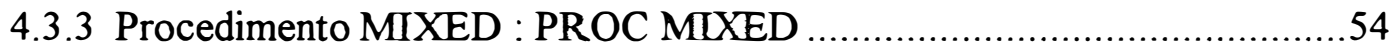

4.3.4 Procedimento MIXED versus procedimento GLM.................................57

4.3.5 Procedimento MIXED versus procedimento VARCOMP ......................58

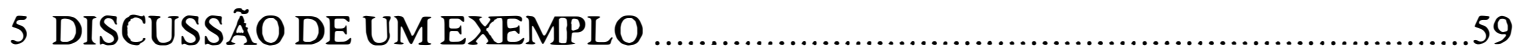

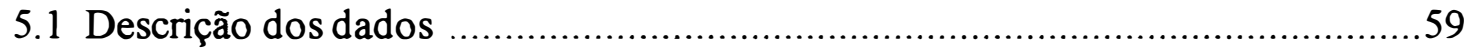

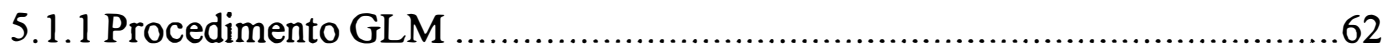

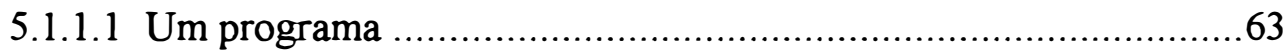

5.1.1.2 Funções estimáveis e hipóteses testáveis ....................................63

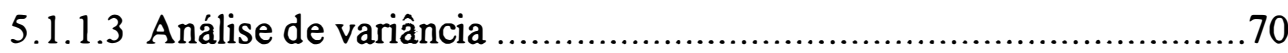

5.1.1.4 Declaração RANDOM / TEST ……...................................... 71

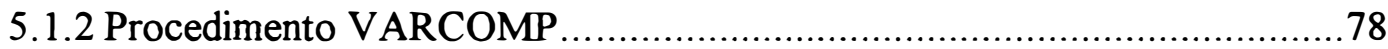

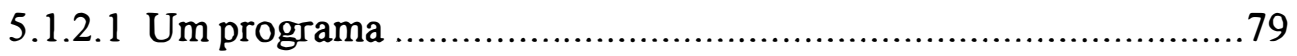

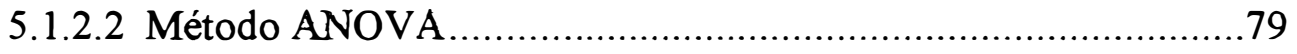

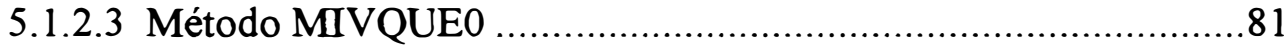

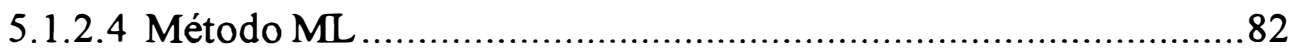

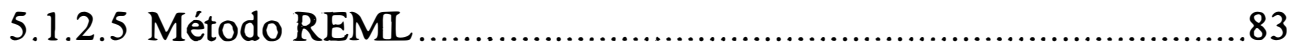

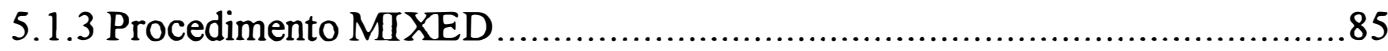

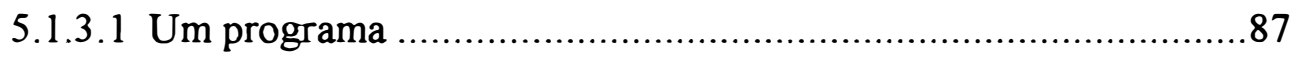

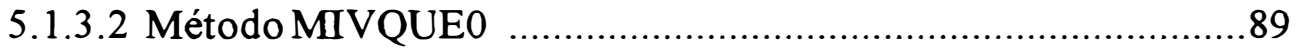

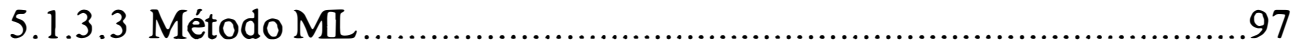

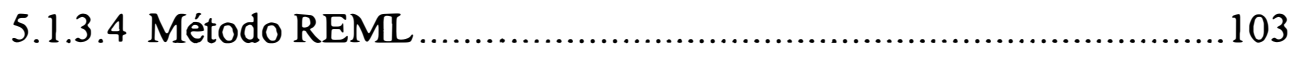

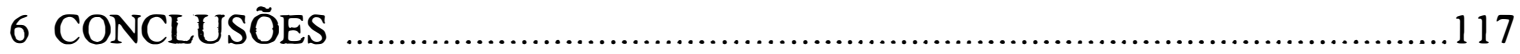

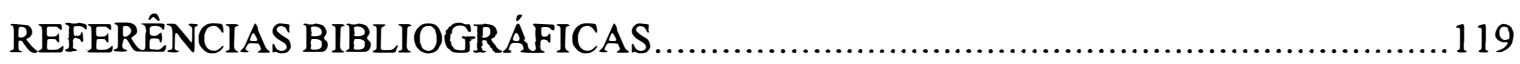




\title{
AJUSTE DE MODELOS MISTOS DE CLASSIFICAÇÃO DUPLA: PROCEDIMENTOS DO SISTEMA ESTATÍSTICO “SAS”
}

\author{
Autora: Sílvia Helena Venturoli Perri \\ Orientador: Prof. Dr. Antonio Francisco Iemma
}

\section{RESUMO}

O Sistema Estatístico SAS apresenta grande flexibilidade para o ajuste de modelos mistos através dos procedimentos abordados GLM, VARCOMP e MIXED, destacando-se a excelente performance do procedimento MIXED.

Embora o PROC MIXED seja o procedimento específico para a análise de modelos mistos, o PROC GLM com a declaração RANDOM e a opção TEST e o PROC VARCOMP com a opção FIXED, também podem ser utilizados.

Uma das vantagens do PROC MIXED consiste em fornecer através da declaração RANDOM e da opção TYPE, várias estruturas para a matriz de variâncias e covariâncias, enquanto que nos procedimentos GLM e VARCOMP, está disponível apenas a estrutura de componentes de variância (VC).

Neste estudo foram adotadas as estruturas VC, CS, TOEP(1) e HF, dentre as quais, segundo os critérios AIC e BIC, apenas a estrutura HF não se mostrou adequada. Este fato foi também observado na estimação dos componentes de variância e nos testes de hipóteses sobre o fator de efeitos fixos. 


\title{
FITTING MIXED MODELS FOR THE TWO-WAY LAYOUT:
}

\section{PROCEDURES OF THE STATISTICAL SOFTWARE "SAS"}

\author{
Author: Sílvia Helena Venturoli Perri \\ Adviser: Prof. Dr. Antonio Francisco Iemma
}

\section{SUMMARY}

The Statistical Software SAS shows large flexibility of fitting mixed models from the GLM, VARCOMP and MIXED procedures, distinguishing the excellent performance of the MIXED procedure.

Although the PROC MIXED is the specific procedure for the analysis of mixed models, the PROC GLM with the RANDOM statement and the TEST option and the PROC VARCOMP with the FIXED option can also be used.

One of the advantage of the PROC MIXED is to provide with the RANDOM statement and the TYPE option, many structures for the variance and covariance matrix, while in the GLM and VARCOMP procedures, only one structure of variance components (VC) is available.

In this work, the VC, CS, TOEP(1) and HF structures were used, among them, according to AIC and BIC criteria, only the HF structure was not appropriate. This fact was also observed in the variance components estimators and in the hypothesis tests on the fixed effects factor. 


\section{INTRODUÇÃO}

A teoria estatística desenvolvida para a análise de dados desbalanceados, por meio de modelos lineares tem sido amplamente estudada, principalmente sob o prisma dos modelos de efeitos fixos. Ocorre, no entanto, em muitas situações experimentais, que um ou mais efeitos podem estar associados a processos de amostragem, pois nem sempre é possivel levar em consideração toda a população. Efeitos desse tipo são, em geral, chamados aleatórios, e caracterizam os modelos de efeitos aleatórios. Quando são estudados simultaneamente efeitos fixos e aleatórios ficam caracterizadrs os modelos mistos.

O modelo misto consiste numa importante classe de modelos que tem sido tradicionalmente analisada por meio de procedimentos da análise de variância. Exemplos de modelos mistos, dentre outros, podem ser obtidos nos delineamentos em blocos aleatórios; nos modelos para experimentos com esquemas fatoriais cruzados ou hierarquizados, com alguns efeitos fixos e outros aleatórios; nos delineamentos com dados desbalanceados com restrição na casualização; em certos delineamentos com medidas repetidas, e assim por diante.

Nos modelos mistos, três aspectos são fundamentais: estimação e testes de hipóteses dos efeitos fixos, predição dos efeitos aleatórios e estimação dos componentes de variância.

$\mathrm{Na}$ análise de um experimento o usuário, em geral, deseja testar hipóteses sobre os efeitos fixos, obter estimativas por ponto e por intervalo para funções estimáveis de interesse e para os componentes de variância. A maior parte deles utiliza softwares 
estatísticos e conseqüentemente é fundamental o conhecimento das principais regras de utilização dos mesmos, nem sempre disponíveis com riqueza de detalhes nos manuais. Assim, por exemplo, as hipóteses sobre os efeitos fixos que estão sendo testadas na análise de variância para modelos mistos desbalanceados nem sempre são bem definidas e claras, dificultando sua correta interpretação e não raro podem induzir o usuário de áreas aplicadas à conclusões incorretas sobre suas pesquisas. 


\section{OBJETIVOS}

Este trabalho tem por objetivos:

1. Apresentar os principais métodos de estimação e de análise, utilizados no estudo de modelos mistos com estruturas gerais de covariâncias nos efeitos aleatórios.

2. Estudar e interpretar as hipóteses, sobre efeitos fixos, que estão sendo testadas na análise de variância de modelos mistos com dois fatores desbalanceados.

3. Ilustrar a utilização dos procedimentos General Linear Models Procedure (PROC GLM), Variance Components Procedure (PROC VARCOMP) e Mixed Procedure (PROC MIXED) do sistema computacional Statistical Analysis System (SAS) versão 6.12 , no ajuste de modelos mistos.

4. Proceder a um estudo comparativo, sem o apelo de competição, entre os procedimentos utilizados pelo SAS para o conjunto de dados em questão.

5. Divulgar o tema entre os profissionais das ciências aplicadas, usuários do modelo misto e de sistemas computacionais estatísticos. 


\section{REVISÃO DE LITERATURA}

Segundo Scheffé (1959), o modelo misto foi amplamente estudado por Fisher ${ }^{1}$ em 1918, com grande repercussão nos estudos de genética quantitativa. Tal modelo foi denominado pelo autor de modelo de componentes de variância.

Dada a importância do tema, diversos métodos têm sido propostos para estimar os componentes de variância, destacando-se os métodos I, II e III de Henderson (Henderson, 1953); o método da máxima verossimilhança (Maximum Likelihood : ML) devido a Hartley \& Rao (' 967); o método da estimação quadrática não-viesada de norma mínima (Minimum Norm Quadratic Unbiased Estimation : MINQUE) amplamente discutido nos artigos de Rao (1970; 1971a; 1972), o método da estimação quadrática não-viesada de variância mínima (Minimum Variance Quadratic Unbiased Estimation : MIVQUE) descrito em Rao (1971b) e o método da máxima verossimilhança restrita (Restricted Maximum Likelihood: REML) descrito por Patterson \& Thompson (1971).

Henderson (1953) propõe três métodos de estimação de componentes de variância para dados desbalanceados de forma descritiva e amplamente ilustrada: os métodos I, II e III de Henderson. Esses três métodos são adaptações do método da análise de variância (ANOVA) para dados balanceados, que consiste em igualar os quadrados médios da análise de variância aos seus valores esperados. O método I é uma modificação do método ANOVA, motivada pelo desbalanceamento dos dados. Esse método consiste em igualar os quadrados médios às suas esperanças matemáticas e na

\footnotetext{
${ }^{1}$ FISHER, RA. The correlation between relatives on the supposition of Mendelian inheritance. Trans. Roy. Soc. Edinburgh, v.52, p.399-433,1918.
} 
resolução do sistema de equações obtido. O método II consiste em estimar, inicialmente, os efeitos fixos e, então aplicar o método I aos resíduos restantes. O método III, também conhecido como método do ajuste de constantes, utiliza as reduções nas somas de quadrados do modelo completo oriundas de parametrizações sucessivas e de submodelos, para estimar os componentes de variância.

Hartley \& Rao (1967) aplicam o método da máxima verossimilhança ao modelo misto geral. A estimação por máxima verossimilhança consiste num método conhecido, desenvolvido por Fisher em $1925^{2}$, e os autores figuram entre os primeiros a aplicá-lo ao modelo misto geral. Esse método consiste em maximizar a função densidade de probabilidade das observações, em relação aos efeitos fixos e aos componentes de variância. Os autores propõem algoritmos para calcular simultaneamente as estimativas de máxima verossimilhança de ambos os componentes, fixos e aleatórios, e discutem propriedades assintóticas de consistência e eficiência dos estimadores.

Searle (1968) faz uma revisão crítica e reformula, na forma de matrizes, a teoria dos métodos de Henderson (1953). Propõe modificações, apresentando algumas conclusões e sugestões:

- O método I é de fácil aplicação, mas não é apropriado para modelos mistos.

- Generaliza o método II e enfatiza que o mesmo não deve ser utilizado em modelos que incluam interações entre efeitos fixos e aleatórios.

- O método III utiliza o método de ajustamento de constantes de Yates $(1934)^{3}$, no qual, a soma de quadrados para cada fator é calculada por meio da diferença entre as somas de quadrados, obtidas pelo ajuste de um modelo completo e de um sub-modelo que exclui aquele fator. Após a obtenção das somas de quadrados, são calculados os quadrados médios que, igualados às suas esperanças matemáticas,

${ }^{2}$ FISHER, R.A. Statistical methods for research workers. Edinburgh: Oliver and Boyd, 1925.

${ }^{3}$ YATES, F. The analysis of multiple classifications with unequal numbers in the different classes. Jour. Amer. Statist. Assoc., v.29, p.51-66, 1934. 
fornecem as estimativas de componentes de variância. Segundo o autor, esse método é apropriado para os modelos mistos.

Cunningham \& Henderson (1968) introduzem um procedimento iterativo para estimar os efeitos fixos e os componentes de variância, em modelos mistos, utilizando-se o método III de Henderson, na sua forma matricial, e o método da máxima verossimilhança. Como a eficiência de processos iterativos depende da qualidade dos valores iniciais dos componentes de variância, eles recomendam usar as estimativas dos componentes de variância obtidas por meio do método III de Henderson, substituindo-se os valores negativos por zero, como estimativas iniciais do procedimento iterativo.

Searle (1970) desenvolve uma expressão geral para os elementos da matriz de informação dos estimadores de máxima verossimilhança, para os componentes de variância para dados desbalanceados, no modelo misto geral. A expressão é utilizada na obtenção de resultados explícitos para o modelo de classificação dupla hierarquizado e no modelo aleatório.

Searle (1971) apresenta uma discussão extensiva das várias formul ções utilizadas para o modelo misto com dois fatores, e discute as vantagens e desvantagens de cada método para estimar componentes de variância. Segundo o autor, em modelos mistos balanceados, os métodos mais usados são ANOVA e ML. O autor apresenta também uma discussão compreensível da estimação dos componentes de variância e da construção de intervalos de confiança exatos e aproximados, para casos em que um estimador pode ser escrito como combinação linear de quadrados médios. Há, no entanto, pouca discussão sobre estimação e testes para efeitos fixos.

Patterson \& Thompson (1971) propõem uma modificação do método da máxima verossimilhança, para delineamentos em blocos e, generalizam este método para qualquer modelo de análise de variância. A idéia consiste na estimação de componentes de variância por máxima verossimilhança, sem considerar os efeitos fixos. Eles dividem a função de verossimilhança em duas partes independentes, uma referente aos efeitos fixos e outra aos aleatórios. A maximização da função de verossimilhança da parte referente aos efeitos aleatórios, fornece os estimadores de máxima verossimilhança para os 
componentes de variância e, a outra fornece os estimadores para os efeitos fixos. Esse método é conhecido como método REML.

Rao (1971a; 1971b; 1972) desenvolve estimadores quadráticos para componentes de variância e para combinações lineares dos mesmos, satisfazendo algumas propriedades: invariância quanto à translação dos efeitos fixos, não-tendenciosidade e norma mínima da diferença entre o estimador e seu verdadeiro valor ou variância mínima do estimador. Os estimadores assim desenvolvidos são chamados de MINQUE (norma mínima) e MIVQUE (variância mínima). Esses métodos baseiam-se na estimação de funções quadráticas dos componentes de variância, por meio de formas quadráticas das observações. O MINQUE utiliza a restrição de que a norma euclidiana da matriz núcleo da forma quadrática seja mínima, e o MIVQUE de que a matriz núcleo seja determinada de forma que os estimadores tenham variância mínima.

Hemmerle \& Hartley (1973) desenvolvem a matriz de transformação W e a utilizam para a obtenção das estimativas de ML, dos componentes de variância e dos efeitos fixos para a análise de variância no modelo misto. Tal procedimento pode ser considerado uma modificação do método de Newton-Raphson, no qual é imposta a restrição de que os componentes de variância sejam não negativos. Nesse artigo sua implementação é discutida com exemplos.

Brown (1976) determina as distribuições assintóticas de dois estimadores de componentes de variância: MINQUE apresentado nas publicações de RAO (1970, 1971a, 1972) e do MINQUE iterativo que é referido como I-MINQUE. O autor informa ainda que ambos os estimadores são consistentes, não exigem suposição sobre a forma da distribuição, contudo, a distribuição assintótica do MINQUE pode depender da escolha da norma enquanto que o I-MINQUE tem uma distribuição assintótica independente da escolha da norma. Sob suposição de normalidade, o I-MINQUE é assintoticamente equivalente ao estimador de máxima verossimilhança e assintoticamente eficiente. $\mathrm{O}$ MINQUE é menos robusto no sentido que estas duas propriedades nem sempre se aplicam. 
Miller (1977) discute e prova as propriedades assintóticas de consistência e normalidade dos estimadores de máxima verossimilhança para o modelo misto, apresentando dois exemplos para ilustrar tais propriedades assintóticas. Ele faz ainda comentários sobre a eficiência assintótica desses estimadores e sobre testes da razão de verossimilhança para o modelo misto, observando-se que os testes da razão de verossimilhança embora fáceis de serem calculados, têm uso limitado pois a distribuição sob a hipótese nula é geralmente desconhecida.

Harville (1977) discute detalhadamente os procedimentos de Máxima Verosimilhança e Máxima Verossimilhança Restrita, na estimação dos componentes de variância e relata os problemas a eles relacionados. $\mathrm{O}$ autor discute o problema de que os estimadores de máxima verossimilhança não levam em consideração a perda de graus de liberdade resultantes da estimação dos efeitos fixos do modelo, problema que foi eliminado por Patterson \& Thompson (1971) com a apresentação do método REML. Outro problema abordado é de que os estimadores de máxima verosimilhança são deduzidos, geralmente, sob a suposição de normalidade, mas, segundo o autor, eles podem ser utilizados quando a forma da distribuição não é especificada. Argumenta, ainda, que no desenvolvimento do MINQUE, nenhuma suposição é feita quanto à distribuição dos efeitos aleatórios, mas sob normalidade desses efeitos o MINQUE, tem variância mínima, ou seja, é um MIVQUE. O autor mostra, ademais, que o método MIVQUE usado iterativamente fornece estimadores idênticos aos estimadores de REML.

Smith \& Hocking (1978) desenvolvem analiticamente a inversa da matriz de covariâncias para o modelo misto geral com dados balanceados e a utilizam na identificação de estatísticas suficientes e no desenvolvimento das equações de verossimilhança.

Ciól (1982) apresenta um estudo sobre os métodos de estimação de componentes de variância: ANOVA, MINQUE, MIVQUE, ML e REML, em modelos lineares mistos, acompanhados de algoritmos para a resolução numérica do problema de estimação e de dois exemplos reais da aplicação desses métodos. 
Giesbrecht (1983) desenvolve um método eficiente para obter as estimativas dos componentes de variância por meio do método MINQUE e estimativas de mínimos quadrados generalizados dos efeitos fixos, no modelo misto. $\mathrm{O}$ algoritmo apresentado utiliza uma modificação da transformação W, proposta por Hemmerle \& Hartley (1973).

Valério Filho (1983) apresenta um estudo elucidando aspectos teóricos sobre os métodos de Henderson, para dados não balanceados, visando facilitar suas aplicações práticas. $\mathrm{O}$ autor conclui que:

- No caso de modelo aleatórios em geral, o método I de Henderson, apropriado para modelos aleatórios, em linhas gerais, é uma extensão do método ANOVA;

- No caso de dados desbalanceado; o método II de Henderson é, na realidade, uma modificação do método I, para que ele possa ser aplicado em modelos mistos que não incluam interações entre efeitos fixos e aleatórios. O método III de Henderson, aplica-se tanto para modelos mistos como para modelos aleatórios, apesar de ser mais utilizado no prim siro caso.

Swallow \& Monahan (1984) apresentam, por meio de simulação, um estudo comparativo entre os métodos de estimação dos componentes de variância: ANOVA, ML, REML, MIVQUE(A) e MIVQUE(0), para o modelo aleatório de classificação simples desbalanceado. Nesse artigo definem o MIVQUE(0) como o MIVQUE com a suposição à priori de que a matriz de variâncias e covariâncias é a matriz identidade e o MIVQUE(A) como o MIVQUE cujas as estimativas à priori são obtidas pelo Método ANOVA. Os autores apresentam algumas conclusões:

- Os estimadores ANOVA apresentam bom desempenho, exceto quando há alto grau de desbalanceamento dos dados e a relação entre as variâncias dos efeitos aleatórios e resíduo é maior do que um;

- O estimador de ML é excelente e o MIVQUE(A) é adequado quando a relação entre as variâncias dos efeitos aleatórios e resíduo é menor do que meio; 
- O MIVQUE(0), default do procedimento VARCOMP do SAS, para se estimar o componente associado ao fator aleatório, tem bom desempenho quando a relação entre as variâncias dos efeitos aleatórios e resíduo é próxima de zero. Contudo, para relações maiores ou iguais a um, este método apresenta um fraco desempenho, tanto para o fator aleatório como para o resíduo;

- Todos os métodos apresentam pouca tendenciosidade para se estimar o componente da variância associado ao resíduo e, em termos de erro quadrático, o MIVQUE(0) é o menos eficaz.

Seraphin (1984) compara por meio de simulação três métodos de estimação dos componentes de variância: MIVQUE, ML e método III de Henderson, em dois modelos com dois fatores: hierárquico aleatório e fatorial misto com interação. Entre as conclusões, destacam-se:

- O MIVQUE e método III de Henderson produzem os mesmos resultados para dados balanceados;

- O ML apresenta bons resultados desde que não se leve em consideração a tendenciosidade;

- Os métodos se mostram mais eficientes, quando a relação entre as variâncias, dos efeitos aleatórios e resíduo, é igual a 0,25 e também quando os dados são balanceados.

Milliken \& Johnson (1984) apresentam, uma versão elucidativa sobre a teoria dos modelos mistos. Eles discutem estimativas por ponto, estimativas por intervalo, e testes de hipóteses sobre ambos os componentes, fixo e aleatório, tanto nos casos balanceados quanto nos desbalanceados.

Hocking (1985) discute os conceitos associados com a análise dos modelos de médias de caselas, especialmente nos casos desbalanceados e também com caselas vazias. Discute também o modelo misto, incluindo testes exatos e aproximados além dos métodos: ANOVA, ML e REML, para estimar os componentes de variância.

Searle (1987) apresenta um sumário da formulação do modelo misto e discute as vantagens e desvantagens dos métodos de estimação: ANOVA, métodos I, II e III de 
Henderson, ML, REML, MINQUE e MIVQUE, nos casos balanceado e desbalanceado. No entanto, como o texto é dedicado aos delineamentos não balanceados de efeitos fixos, há pouca discussão sobre testes de hipóteses e sobre estimação dos componentes fixos no modelo misto.

Christensen (1987) discute brevemente o modelo misto geral e indica que o BLUE pode ser obtido através do método dos mínimos quadrados generalizados desde que a matriz de covariâncias seja conhecida. Discute também testes de hipóteses usando formulação matricial, mas não discute testes aproximados. Apresenta uma discussão compacta de estimação dos componentes de variância em modelos não balanceados, mas discute somente o método ANOVA.

Valério Filho (1991) apresenta um estudo comparativo, por meio de simulação de dados, entre os métodos para estimação de componentes de variância disponíveis no SAS VARCOMP: ANOVA, ML, REML, MIVQUE(0). O autor utiliza tal procedimento em dois tipos de modelos mistos com dois fatores sem interação: o cruzado e o hierárquico. Destacam-se as seguintes conclusões:

- Com dados balanceados os métodos ANOVA, REML e MIVQUE(0) são equivalentes;

- Com dados desbalanceados o desempenho de cada método depende, entre outros, do modelo, do componente e do próprio critério utilizado na comparação. Mas de uma forma geral o método ML e principalmente o REML, apresentam freqüentemente os melhores resultados enquanto que, o método MIVQUE(0) raramente conseguiu superar os demais. O método ANOVA foi superior em alguns casos, mas em geral, esteve próximo dos métodos ML e REML e mostrando mais qualidades do que o método MIVQUE(0).

Searle et al. (1992) apresentam uma ampla discussão sobre: estimação de componentes de variância e análise de modelos mistos, ilustrando-os por meio de exemplos. Apresentam um histórico da estimação dos componentes de variância e descrevem, em detalhes, os métodos de estimação: ANOVA, métodos I, II e III de 
Henderson, ML e REML, dos componentes de variância, bem como a predição de efeitos aleatórios.

Barbin (1993) apresenta um trabalho sobre componentes de variância enfatizando, o método ANOVA, para modelos balanceados. Apresenta, também, um método prático, para a obtenção dos componentes de variância, nos modelos balanceados, e sugere uma modificação nesse método, visando à coerência entre os resultados por ele obtidos e aqueles obtidos por meio do método dedutivo.

Schwarz (1993) apresenta um breve resumo sobre testes de hipóteses e inferência em modelos mistos balanceados e estuda a performance dos sistemas computacionais: BMDP, versão 1990; SAS, versão 6 e SPSS, versão 4.0 quando utilizados para analisar os modelos mistos balanceados.

Wolfinger (1993) descreve medidas estatísticas para comparar várias estruturas de covariâncias em modelos mistos. A seleção dessas estruturas envolve a utilização do critério de informação de máxima verossimilhança e máxima verosimilhança restrita. A seleção de modelos para estruturas de covariâncias em modelos mistos é ilustrada por meio de dois exemplos.

Richardson \& Welsh (1995) apresentam duas definições de máxima verossimilhança robusta e máxima verossimilhança restrita robusta e as utiliza em dados de experimentos biológicos e químicos. Apresentam também, por meio de simulação, um estudo para investigar as propriedades assintóticas e para examinar as vantagens de utilizar esses métodos robustos. Esses métodos são robustos no sentido de que visam limitar a influência de observações outliers sobre as estimativas. Os autores concluem que as estimativas dos componentes de variância obtidas por esses métodos robustos são sempre menores que as estimativas clássicas.

Gilmour et al. (1995) descrevem e aplicam o algoritmo AI, Average Information, para estimar componentes de variância pelo método da máxima verossimilhança restrita, em modelos mistos com erros correlacionados. Esse algoritmo apresenta propriedades de convergência similares ao algoritmo FS, Fisher Scoring, e como o algoritmo FS, ele pode ser usado para estimar componentes de variância negativos. Os autores mostram que para 
grandes conjuntos de dados, geralmente encontrados nas áreas de melhoramento animal e vegetal, o algoritmo AI constitui uma alternativa viável aos algoritmos DF, Derivative Free, e EM, Expectation-Maximization, além de apresentar a vantagem de convergir com um número menor de iterações. 


\section{METODOLOGIA}

Modelos de efeitos fixos são utilizados para descrever dados de experimentos cuja estrutura de tratamentos envolve apenas fatores fixos, com exceção do erro que é tomado como aleatório.

Nos modelos de efeitos fixos tem-se, em geral, interesse na obtenção de estimativas por ponto, por intervalo e por região, bem como nos testes de hipóteses estatísticas sobre os fatores fixos e seus níveis.

Modelos misto- são utilizados para descrever dados de experimentos cuja estrutura de tratamentos envolve alguns fatores que são fixos e alguns que são aleatórios, ou seja modelos que contêm efeitos fixos e aleatórios, independentemente da média e do erro. Assim esses modelos envolvem duas partes, uma parte descrevendo os efeitos aleatórios e a outra descrevendo os efeitos fixos. Consequentemente, a análise de um modelo misto pode ser posta em duas partes: uma análise para a parte aleatória e outra para a parte fixa.

Nos modelos mistos a análise da parte aleatória consiste de estimação e testes de hipóteses sobre os componentes de variância. A análise da parte fixa consiste de estimação e testes de hipóteses sobre funções estimáveis dos efeitos fixos. A análise dos modelos mistos é, em geral, iniciada com a parte aleatória.

Seguindo a metodologia adotada por Searle (1971; 1987), Henderson (1984),

Searle et al. (1992) e Iemma (1987; 1993), são abordadas as hipóteses estatísticas sobre efeitos fixos e as somas de quadrados a elas associadas, considerando-se os modelos mistos de classificação dupla bem como os principais métodos de estimação dos 
componentes de variância. Ademais, são discutidos os comportamentos de alguns procedimentos do sistema estatístico SAS utilizados para a análise de modelos mistos.

\subsection{Modelo linear fixo}

O modelo linear de efeitos fixos é, aqui, denotado por

$$
\mathrm{y}=\mathbf{X} \theta+e
$$

onde :

y é o vetor de observações, de dimensão nxl,

$\mathbf{X}$ é a matriz conhecida do planejamento, de dimensão nxp,

$\theta$ é o vetor de efeitos fixos, de dimensão pxl,

$e$ é o vetor de erros aleatórios não observáveis, de dimensão $\mathrm{nxl}$, com estrutura de Gauss-Markov Normal, ou seja $e \sim \mathrm{N}\left(\phi, \mathbf{I}^{2}\right)$.

Segundo Searle (1987), o modelo linear é superparametrizado quando apresenta explicitamente um parâmetro para cada efeito envolvido. Conforme Iemma (1993) o modelo superparametrizado é excelente para a boa interpretação da notação $R(\cdot)$ e de certas somas de quadrados a ela associadas.

\subsubsection{Estimação}

Para o modelo linear de efeitos fixos, definido por $\mathrm{y}=\mathrm{X} \theta+e \operatorname{com} e \sim \mathrm{N}\left(\phi, \mathbf{I} \sigma^{2}\right)$, entre os métodos de estimação disponíveis na literatura, o mais utilizado tem sido o de mínimos quadrados. Esse método conduz ao sistema de equações normais $\mathbf{X}^{\mathbf{}} \mathbf{X} \boldsymbol{\theta}=\mathbf{X}^{\mathbf{X}} \mathbf{Y}$. 
Segundo Iemma (1991), se a matriz $\mathbf{X}$ é de posto coluna completo então $\mathbf{X}^{\prime} \mathbf{X}$ é positiva definida e portanto não singular. Nesse caso o sistema consistente $\mathbf{X}^{\prime} \mathbf{X} \theta=\mathbf{X}^{\prime} \mathbf{y}$ tem solução única dada por $\hat{\theta}=\left(\mathbf{X}^{\prime} \mathbf{X}\right)^{-1} \mathbf{X}^{\prime}$ y. Essa solução única, é a solução aproximada de mínimos quadrados para o sistema inconsistente $\mathrm{y}=\mathbf{X} \theta \mathrm{e}$, coincide com o estimador de mínimos quadrados para $\theta$. Isto nos garante que cada componente do vetor $\theta$ é estimável e portanto testável. Por outro lado, se $\mathbf{X}$ não é de posto coluna completo, então $\mathbf{X}^{\prime} \mathbf{X}$ é singular e o sistema de equações normais, sempre consistente, é indeterminado. Suas soluções exatas podem ser obtidas entre outras, por $\theta^{0}=\mathbf{X}^{1} \mathbf{y}=\left(\mathbf{X}^{\prime} \mathbf{X}\right)^{\mathrm{g}} \mathbf{X}^{\prime} \mathbf{y}$; onde $\mathbf{X}^{1}$ é uma inversa generalizada de mínimos quadrados para $\mathbf{X}$ e $\left(\mathbf{X}^{\mathbf{X}} \mathbf{X}\right)^{\mathfrak{g}}$ é uma inversa generalizada qualquer de $\mathbf{X}^{\prime} \mathbf{X}$, e são soluções aproximadas de mínimos quadrados para $\mathbf{y}=\mathbf{X} \theta$ inconsistente.

Segundo Rao (1945), uma função linear paramétrica $\lambda^{\prime} \theta$ é estimável no modelo linear de Gauss-Markov, se e somente se $\lambda \in C\left(\mathbf{X}^{\prime}\right)$. Para fins práticos, no entanto, é bom não perder de vista que:

a) $E[y]=X \theta$ e então $\mathbf{X} \theta$ é estimável;

b) Combinações lineares de funções estimáveis são também estimáveis.

Com essas duas regras práticas pode-se resolver grande parte do problema da estimabilidade, a menos de desconexões.

Ademais, pelo teorema de Gauss-Markov, se $\lambda^{\prime} \theta$ é estimável então seu BLUE (Best Linear Unbiased Estimator) é dado de modo único por $\widehat{\lambda^{\prime} \theta}=\lambda^{\prime} \theta^{0}$, onde $\theta^{0}$ é qualquer solução das equações normais.

Fatos importantes sobre os quatro tipos de estimadores por ponto, sob o ponto de vista do sistema estatístico SAS, podem ser obtidos em Iemma (1991), Iemma e Perri (1997), Mondardo e Iemma (1998) entre outros.

A estimação por intervalo ao nível de confiança $1-\alpha$ é feita por meio de

$$
\operatorname{IC}\left[\lambda^{\prime} \theta\right]_{1-\alpha}: \quad \lambda^{\prime} \theta^{0} \pm \mathrm{t}_{\{\mathrm{n}-\mathrm{r}[\mathrm{x}] ; \alpha / 2\}} \sqrt{\widehat{\widehat{\mathrm{VAR}}}\left[\lambda^{\prime} \theta^{0}\right]}
$$


e o elipsóide de confiança para a estimação por região para um conjunto de $\mathrm{k}$ funções estimáveis linearmente independentes, ao nível de confiança $1-\alpha$, é

$$
R\left[B^{\prime} \theta\right]:\left(B^{\prime} \theta-B^{\prime} \theta^{0}\right)^{\prime}\left[B^{\prime}\left(X^{\prime} \mathbf{X}\right)^{g} B\right]^{-1}\left(B^{\prime} \theta-B^{\prime} \theta^{0}\right) \leq k F \text { QMRes }
$$

onde $\lambda^{\prime} \theta$ e $B^{\prime} \theta$ são estimáveis e, por construção, $B^{\prime}$ tem posto linha completo $k$.

\subsubsection{Testes de hipóteses}

$\mathrm{O}$ vetor $\hat{\mathrm{y}}=\mathbf{X} \theta^{0}$ é a aproximação de mínimos quadrados para o vetor y das observações, invariante para qualquer $\theta^{0}$ solução das equações normais. Assim,

$$
\hat{\mathbf{y}}=\mathbf{X}\left(\mathbf{X}^{\prime} \mathbf{X}\right)^{\mathrm{g}} \mathbf{X}^{\prime} \mathbf{y}=\mathrm{Py}
$$

onde $\mathbf{P}=\mathbf{X}\left(\mathbf{X}^{\prime} \mathbf{X}\right)^{\mathrm{g}} \mathbf{X}^{\prime}=\mathbf{X X}^{+}=\mathbf{X} \mathbf{X}^{1}$ é o projetor ortogonal de y sobre o espaço gerado pelas colunas de $\mathbf{X}, C(\mathbf{X})$ e $\mathbf{X}^{+}$é a inversa generalizada de Moore-Penrose.

Geometricamente

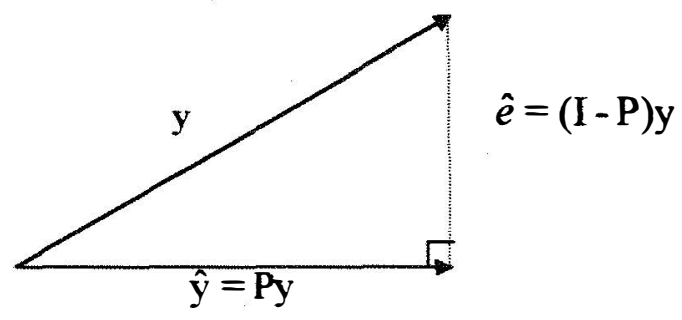

$C(\mathbf{X})$

onde $\mathrm{y}=\hat{\mathrm{y}}+\hat{\mathrm{e}}, \mathrm{y} \in \mathrm{R}^{\mathrm{n}}, \hat{\mathrm{y}} \in C(\mathbf{X}), \hat{\mathrm{e}} \in C^{\perp}(\mathbf{X})$ e $\mathrm{R}^{\mathrm{n}}=C(\mathbf{X}) \oplus C^{\perp}(\mathbf{X})$.

Aqui I - P é o projetor ortogonal de y sobre o complemento ortogonal do espaço coluna de $\mathbf{X}, C^{\perp}(\mathbf{X})$. 
Dada a ortogonalidade pode-se utilizar o teorema de Pitágoras e obter-se a decomposição ortogonal clássica da análise de variância:

$$
\|\mathrm{y}\|^{2}=\|\hat{y}\|^{2}+\|\hat{e}\|^{2}
$$

onde

$$
\begin{aligned}
& \|\mathrm{y}\|^{2}=y^{\prime} y=\sum y^{2}=S . Q . T o t a l \\
& \|\hat{y}\|^{2}=\hat{y}{ }^{\prime} \hat{y}=\sum \hat{y}^{2}=y^{\prime} P y=\theta^{0}{ }^{\prime} X^{\prime} y=S Q \text { devida aos Parâmetros } \\
& \|\hat{e}\|^{2}=\hat{e}^{\prime} \hat{e}=\sum \hat{e}^{2}=y^{\prime}(I-P) y=y^{\prime} y-\theta^{0} X^{\prime} X^{\prime} y=\text { SQ Residual }
\end{aligned}
$$

Searle (1971) e Iemma (1987), entre outros, adotam a estatística de Wald como um procedimento alternativo muito útil para obter as somas de quadrados das hipóteses de interesse. Assim, para a hipótese $\mathrm{H}_{0}: \mathrm{B}^{\prime} \theta=\phi$, onde $\mathrm{B}^{\prime}$ tem posto linha completo, tem-se:

$$
\text { SQ } \mathrm{H}_{0}=\left(\mathrm{B}^{\prime} \theta^{0}\right)^{\prime}\left[\mathrm{B}^{\prime}\left(\mathbf{X}^{\prime} \mathbf{X}\right)^{g} \mathrm{~B}\right]^{-1}\left(\mathrm{~B}^{\prime} \theta^{0}\right)
$$

verifica-se também, que

$$
\frac{\mathrm{SQH}_{0}}{\sigma^{2}} \sim \chi_{\{r[\mathrm{~B}] ; 0\}}^{2}
$$

onde $\delta=\frac{1}{2 \sigma^{2}} \theta^{\prime} \mathbf{X}^{\prime} \mathrm{P}^{*} \mathbf{X} \theta$ é o parâmetro de não centralidade e $\mathrm{P}^{*}$ é um projetor ortogonal tal que $y^{\prime} \mathrm{P}^{*} \mathrm{y}=\mathrm{SQH}_{0}$. Ademais, sob normalidade, se $\mathrm{P}^{*}\left(\mathrm{I}-\mathrm{P}^{*}\right)=\phi$ então as formas quadráticas associadas são independentes e o quociente dos respectivos quadrados médios tem distribuição de $\mathrm{F}$ não central

$$
\frac{\frac{\mathrm{QMH}_{0}}{\sigma^{2}}}{\frac{\mathrm{QMRes}}{\sigma^{2}}} \sim \mathrm{F}_{\{[\mathrm{B}[\mathrm{Br}[\mathrm{I}[\mathrm{P}] ; \delta\}}
$$

e

$$
\mathrm{E}\left[\mathrm{SQH}_{0}\right]=\mathrm{E}\left[\mathrm{y}^{\prime} \mathrm{P}^{*} \mathrm{y}\right]=\operatorname{Tr}\left[\mathrm{P}^{*}\right] \sigma^{2}+\theta^{\prime} \mathbf{X}^{\prime} \mathrm{P}^{*} \mathbf{X} \theta
$$

Desse modo, com base no parâmetro de não centralidade $\delta$ ou na esperança da $\mathrm{SQH}_{0}$ pode-se verificar qual é a verdadeira hipótese que está sendo testada. 


\subsection{Modelo linear misto}

Neste estudo, adota-se, para o modelo linear misto, a forma matricial, descrita em Searle (1987), Searle et al. (1992), entre outros:

$$
\mathrm{y}=\mathbf{X} \theta+\mathbf{Z u}+e
$$

onde:

y é o vetor de observações, de dimensão nxl,

$\mathbf{X}$ é a matriz, conhecida, do planejamento dos efeitos fixos, de dimensão nxp,

$\theta$ é o vetor de efeitos fixos, de dimensão pxl,

$\mathbf{Z}$ é a matriz, conhecida, do planejamento dos efeitos aleatórios, de dimensão nxq, u é o vetor de efeitos aleatórios, de dimensão qxl,

$e$ é o vetor de erros aleatórios não observáveis, de dimensão $\mathrm{nxl}$, com estrutura de Gauss-Markov Normal, ou seja $e \sim \mathrm{N}\left(\phi, \mathbf{I} \sigma_{e}^{2}\right)$.

Particionando u em r sub-vetores de dimensão $\mathrm{q}_{\mathrm{i}} \times 1$, tal que $\sum_{\mathrm{i}=1}^{r} \mathrm{q}_{\mathrm{i}}=\mathrm{q}$, tem-se $\mathrm{u}^{\prime}=\left[\mathrm{u}_{1}^{\prime}, \mathrm{u}_{2}^{\prime}, \cdots, \mathrm{u}_{\mathrm{i}}^{\prime}, \cdots, \mathrm{u}_{\mathrm{r}}^{\prime}\right]$, onde cada vetor $\mathrm{u}_{\mathrm{i}}$ corresponde aos níveis observados do efeito aleatório i.

As pressuposições gerais sobre a estrutura dos valores esperados, variâncias e covariâncias aqui adotadas, são:

$$
\begin{array}{ll}
E(y)=\mathbf{X} \theta & \\
E(u)=0, & \operatorname{var}(\mathrm{u})=\mathbf{G} \\
\mathrm{E}(\mathrm{e})=0, & \operatorname{var}(\mathrm{e})=\mathbf{R}=\mathbf{I} \sigma_{e}^{2} \\
\operatorname{cov}\left(\mathrm{u}, \mathrm{e}^{\prime}\right)=0 &
\end{array}
$$

Logo,

$$
\begin{aligned}
& \mathbf{V}=\operatorname{var}(\mathrm{y})=\operatorname{var}(\mathbf{X} \theta+\mathbf{Z u}+\mathrm{e})=\mathbf{Z} \operatorname{var}(\mathrm{u}) \mathbf{Z}^{\prime}+\mathbf{I} \sigma_{e}^{2} \\
& \mathbf{V}=\mathbf{Z G Z}^{\prime}+\mathbf{I} \sigma_{e}^{2}
\end{aligned}
$$


Assim, tem-se:

$$
\mathrm{E}\left[\begin{array}{l}
\mathrm{u} \\
\mathrm{e}
\end{array}\right]=\left[\begin{array}{l}
0 \\
0
\end{array}\right] \quad \text { e } \quad \operatorname{var}\left[\begin{array}{l}
\mathrm{u} \\
\mathrm{e}
\end{array}\right]=\left[\begin{array}{cc}
\mathbf{G} & 0 \\
0 & \mathbf{I} \sigma_{e}^{2}
\end{array}\right]
$$

Em geral a matriz de covariâncias de y é uma função linear de parâmetros desconhecidos a serem estimados. Assim, quanto mais adequada for a matriz $G$, escolhida à priori, melhores serão as estimativas de $\theta$ e de $u$.

\subsubsection{Predição dos efeitos aleatórios}

No modelo linear misto $\mathrm{y}=\mathbf{X} \theta+\mathbf{Z u}+\boldsymbol{e}$, o vetor $\mathrm{u}$ é um conjunto de valores de variáveis aleatórias, que usualmente não podem ser medidas. Uma questão de freqüente interesse é: "Dado o vetor de dados y, como se pode predizer os valores do vetor u que poderiam estar a ele associados?" Ou, colocado-se de outra maneira, "De todas as possíveis realizações de $u$ que poderiam estar associadas com o particular valor de y observado, qual é um estimador da média, ou valor esperado, desses valores de u?" Em outras palavras, "Qual é "um" estimador da média condicional E(u | y)?"

$\mathrm{O}$ interesse em $\mathrm{E}(\mathrm{u} \mid \mathrm{y})$, pode também ser motivado mais formalmente. Como u e y são (realizações de) variáveis aleatórias, qualquer que seja a distribuição conjunta, pode ser mostrado que o preditor de u, que minimiza o quadrado médio dos erros de predição, é E(u | y), (Cochran, 1951; Rao, 1965; Searle, 1974). Assim E(u | y) é dito o melhor preditor de u. Sob normalidade tem-se a forma

$$
\mathrm{E}(\mathrm{u} \mid \mathrm{y})=\tilde{u}=E(u)+\operatorname{cov}\left(u, y^{\prime}\right)[\operatorname{var}(y)]^{-1}[y-E(y)]
$$

Assim $\widetilde{u}$ é considerado o melhor preditor linear de u sob normalidade. 
No caso do modelo misto $\mathrm{y}=\mathbf{X} \theta+\mathbf{Z u}+e$, com

$$
\begin{aligned}
& \operatorname{var}(\mathrm{u})=\mathbf{G} \quad \text { e } \quad \operatorname{cov}\left(\mathrm{u}^{\prime} \mathrm{y}^{\prime}\right)=\operatorname{cov}\left(\mathrm{u}^{\prime} \mathrm{u}^{\prime} \mathbf{Z}^{\prime}\right)=\mathbf{G} \mathbf{Z}^{\prime} \\
& \operatorname{var}(\mathrm{y})=\mathbf{Z G Z} \mathbf{Z}^{\prime}+\mathbf{I} \sigma_{e}^{2}=\mathbf{V}
\end{aligned}
$$

tem-se

$$
\tilde{\boldsymbol{u}}=\mathbf{G} \mathbf{Z}^{\prime} \mathbf{V}^{-1}(\boldsymbol{y}-\mathbf{X} \theta)
$$

Portanto $\widetilde{u}$ é uma função estimável de $\mathrm{X} \theta$ e assim tem-se

e

$$
\begin{aligned}
& \operatorname{BLUE}(\tilde{\boldsymbol{u}})=\mathbf{G} \mathbf{Z}^{\prime} \mathbf{V}^{-1}\left(y-\mathbf{X} \theta^{0}\right) \\
& \operatorname{BLUE}(\mathbf{X} \theta)=\widehat{\mathbf{X}} \theta=\mathbf{X} \theta^{0}=\mathbf{X}\left(\mathbf{X}^{\prime} \mathbf{V}^{-1} \mathbf{X}\right)^{g} \mathbf{X}^{\prime} \mathbf{V}^{-1} y
\end{aligned}
$$

onde $\mathbf{X} \theta^{0}$ é o BLUE de $\mathbf{X} \theta$, a parte fixa do modelo.

Combinando os conceitos de predição e de melhor estimador linear não-viesado, tem-se o que freqüentemente é referido como o melhor preditor linear não-viesado: BLUP (Best Linear Unbiased Predictor).

Então:

$$
\operatorname{BLUP}(\mathrm{u})=\mathbf{G Z} \cdot \mathbf{V}^{-\mathbf{1}}\left(y-\mathbf{X} \theta^{0}\right)
$$

Quando G e $\sigma^{2}$ são conhecidas o cálculo do BLUP(u) não apresenta dificuldades, a menos de questões numéricas relacionadas a problemas de inversão de matrizes de alta ordem, como pode ocorrer no caso da matriz $\mathbf{V}$, de dimensão igual ao número de observações. No entanto, é possível reduzir essa dificuldade usando-se as "equações do modelo misto", deduzidas inicialmente por Henderson (1973), dadas por:

$$
\left[\begin{array}{cc}
\mathbf{X}^{\prime} \mathbf{X} & \mathbf{X}^{\prime} \mathbf{Z} \\
\mathbf{Z}^{\prime} \mathbf{X} & \mathbf{Z}^{\prime} \mathbf{Z}+\sigma_{e}^{2} \mathbf{G}^{-\mathbf{1}}
\end{array}\right]\left[\begin{array}{c}
\theta^{0} \\
\widetilde{\mathbf{u}}
\end{array}\right]=\left[\begin{array}{c}
\mathbf{X}^{\prime} \mathbf{y} \\
\mathbf{Z}^{\prime} \mathbf{y}
\end{array}\right]
$$

Resolvendo-se esse sistema de equações, obtém-se as soluções para os efeitos fixos $\theta^{0}$, e predições para os efeitos aleatórios $\widetilde{\boldsymbol{u}}$.

Henderson et al. (1959), mostram que $\theta^{0}$ e $\tilde{u}$ de (7) são de fato $\theta^{0}$ de (5) e BLUP(u) de (6). Para isso definem $\mathbf{V}$, matriz de variâncias e covariâncias de $y$, como em (3).

$$
\mathbf{V}=\mathbf{Z G Z}+\mathbf{I} \sigma_{e}^{2} \quad \text { e obtêm } \quad \mathbf{V}^{-1}=\left[\mathbf{I}-\mathbf{Z}\left(\mathbf{Z}^{\prime} \mathbf{Z}+\sigma_{e}^{2} \mathbf{G}^{-\mathbf{1}}\right)^{-1} \mathbf{Z}^{\prime}\right] \sigma_{e}^{-2}
$$


Comparando-se as expressões dadas em (5) e (6) para $\mathbf{X} \theta^{0}$ e $\operatorname{BLUP(u),~a~}$ vantagem da expressão (7) está na matriz a ser invertida pois em (5), a matriz a ser invertida, $\mathbf{V}$, é de ordem $n$, enquanto que em (7), a matriz é de ordem $(p+q)$, onde $p$ e $q$ são os números de parâmetros fixos e aleatórios, respectivamente, e $(p+q)$ é freqüentemente, bem menor do que n, o número de observações.

Segundo os autores, para o desenvolvimento precedente, assume-se que $\mathbf{V}$ é conhecida. Quando V não é conhecida, seus elementos devem ser estimados, ou seja, deve-se estimar os componentes de variância, utilizando-se um dos métodos disponíveis na literatura. Então em (5), e (6) substituindo $\mathbf{V}$ por $\hat{\mathbf{V}}$, tem-se respectivamente

$$
\begin{aligned}
& \widehat{\mathbf{X}} \hat{\theta}_{\hat{\mathrm{v}}}=\mathbf{X} \theta_{\hat{\mathbf{v}}}^{0}=\mathbf{X}\left(\mathbf{X}^{\prime} \hat{\mathbf{V}}^{-1} \mathbf{X}\right)^{g} \mathbf{X}^{\prime} \hat{\mathbf{V}}^{-1} \mathbf{y} \\
& \text { e } \\
& \operatorname{BLUP}(\mathrm{u})_{\hat{\mathbf{v}}}=\hat{\mathbf{G}} \mathbf{Z}^{\prime} \hat{\mathbf{V}}^{-1}\left(y-\mathbf{X} \theta_{\hat{\mathbf{v}}}^{0}\right)
\end{aligned}
$$

Assim

$$
\left[\begin{array}{c}
\theta^{0} \\
\tilde{\mathbf{u}}
\end{array}\right]=\left[\begin{array}{c}
\left(\mathbf{X}^{\prime} \hat{\mathbf{V}}^{-1} \mathbf{X}\right)^{g} \mathbf{X}^{\prime} \hat{\mathbf{V}}^{-1} \mathbf{y} \\
\hat{\mathbf{G}} \mathbf{Z}^{\prime} \hat{\mathbf{V}}^{-1}\left(\mathbf{y}-\mathbf{X} \hat{\theta}_{\hat{\mathbf{v}}}^{0}\right)
\end{array}\right]
$$

Em (7) substituindo $\sigma_{e}^{2}$ e $\mathbf{G}$ por $\hat{\sigma}_{e}^{2}$ e $\hat{\mathbf{G}}$, respectivamente, tem-se

$$
\left[\begin{array}{cc}
\mathbf{X}^{\prime} \mathbf{X} & \mathbf{X}^{\prime} \mathbf{Z} \\
\mathbf{Z}^{\prime} \mathbf{X} & \mathbf{Z}^{\prime} \mathbf{Z}+\hat{\sigma}_{e}^{2} \hat{\mathbf{G}}^{-\mathbf{1}}
\end{array}\right]\left[\begin{array}{c}
\theta^{0} \\
\tilde{\mathrm{u}}
\end{array}\right]=\left[\begin{array}{c}
\mathbf{X}^{\prime} \mathbf{y} \\
\mathbf{Z}^{\prime} \mathbf{y}
\end{array}\right]
$$

Assim

$$
\left[\begin{array}{c}
\theta^{0} \\
\tilde{\mathbf{u}}
\end{array}\right]=\hat{\mathbf{C}}\left[\begin{array}{l}
\mathbf{X}^{\prime} \mathbf{y} \\
\mathbf{Z}^{\prime} \mathbf{y}
\end{array}\right]
$$

onde

$$
\hat{\mathbf{C}}=\left[\begin{array}{cc}
\mathbf{X}^{\prime} \mathbf{X} & \mathbf{X}^{\prime} \mathbf{Z} \\
\mathbf{Z}^{\prime} \mathbf{X} & \mathbf{Z}^{\prime} \mathbf{Z}+\hat{\sigma}_{e}^{2} \hat{\mathbf{G}}^{-\mathbf{1}}
\end{array}\right]^{g}
$$

ou seja, $\hat{\mathbf{C}}$ é uma matriz inversa generalizada dos coeficientes das "equações do modelo misto" (7).

Assim, utilizando-se a expressão (10) ou as expressões (8) e (9) obtém-se as estimativas dos efeitos fixos $\mathrm{e}$ as predições dos efeitos aleatórios, $\theta^{0}$ e $\tilde{\boldsymbol{u}}$, 
respectivamente, com vantagem para a expressão (10) pela menor dimensão da matriz a ser invertida.

Um aspecto interessante das "equações do modelo misto" é que elas podem ser utilizadas em procedimentos iterativos para os cálculos da estimativas ML e REML dos componentes de variância. Maiores detalhes do relacionamento entre essas equações e as estimativas ML e REML são apresentadas em Harville (1977) e Searle et al. (1992).

\subsubsection{Estimação dos componentes de variância}

Conforme já comentado em 4.2.1 para utilizar a definição dada em (9), ou seja, para a obtenção do BLUP de u, exige-se o conhecimento das estimativas dos romponentes de variância.

Estimação dos componentes de variância para dados balanceados é, em geral, feita pelo método da análise de variância, ANOVA. Por esse método obtém-se estimadores igualando-se as somas de quadrados, ou os quadrados médios, de um quadro de análise de variância aos seus respectivos valores esperados, substituindo-se os componentes por suas estimativas, que são combinações lineares dos componentes de variância. Portanto o método ANOVA produz equações lineares nos componentes de variância que são resolvidas e as soluções são tomadas como os estimadores (Barbin, 1993).

Um problema relacionado com a estimação dos componentes de variância para dados desbalanceados consiste no fato de que muitos métodos de estimação estão disponíveis e escolher um deles pode não ser uma questão tão simples (Seraphin, 1984; Lopes et al., 1993). 
Face aos objetivos deste estudo, são aqui estudados os seguintes métodos: ANOVA, métodos I, II e III de Henderson, ML, REML, MINQUE, I-MINQUE, MIVQUE, MIVQUE0 e MIVQUEA.

\subsubsection{Método da análise de variância : ANOVA}

O princípio do método ANOVA muito útil com dados balanceados, que consiste, como já dito em 4.2.2, em igualar somas de quadrados aos seus valores esperados, pode ser generalizado. Para a generalização utiliza-se uma forma quadrática em lugar das somas de quadrados.

Para o modelo (2), seja $\sigma^{2}$ o vetor dos componentes de variância que serão estimados e seja $s$ um retor, da mesma ordem de $\sigma^{2}$, de qualquer forma quadrática linearmente independente das observações.

Suponha que $s$ é tal que

$$
s=\left\{s_{i}\right\}_{i=0}^{r}=\left\{\mathrm{y}^{\prime} \mathbf{A}_{i} \mathrm{y}\right\}_{i=0}^{r}
$$

Então

$$
\begin{aligned}
\mathrm{E}\left(\boldsymbol{s}_{i}\right)=\mathrm{E}\left(\mathrm{y}^{\prime} \mathbf{A y}\right) & =\operatorname{tr}\left(\mathbf{A}_{\mathrm{i}} \mathbf{V}\right)+\mathrm{E}\left(\mathrm{y}^{\prime}\right) \mathbf{A}_{\mathrm{i}} \mathrm{E}(\mathrm{y}) \\
& =\operatorname{tr}\left(\mathbf{A}_{\mathbf{i}} \mathbf{V}\right)+\theta^{\prime} \mathbf{X}^{\prime} \mathbf{A}_{\mathbf{i}} \mathbf{X} \theta
\end{aligned}
$$

Esta esperança não conterá termos em $\theta$, os efeitos fixos se $\mathbf{X}^{\prime} \mathbf{A}_{\mathbf{i}} \mathbf{X}=0$. Assim, deve ser escolhida $\mathbf{A}$ tal que $\mathbf{X}^{\prime} \mathbf{A}_{\mathbf{i}} \mathbf{X}=0$. Então

$$
\mathrm{E}\left(\boldsymbol{s}_{i}\right)=\operatorname{tr}\left(\mathbf{A}_{\mathrm{i}} \mathbf{V}\right)=\operatorname{tr}\left[\mathbf{A}_{i} \sum_{j=0}^{r} \mathbf{Z}_{j} \mathbf{Z}_{j}^{\prime} \sigma_{j}^{2}\right]=\sum_{j=0}^{r} \operatorname{tr}\left(\mathbf{Z}_{j}^{\prime} \mathbf{A}_{i} \mathbf{Z}_{j}\right) \sigma_{j}^{2}
$$

que pode ser reescrito como

$$
\mathrm{E}(s)=\mathbf{D} \sigma^{2}
$$

para alguma matriz $\mathbf{D}$, tal que $\mathbf{D}=\left\{\operatorname{tr}\left(\mathbf{Z}_{j} \mathbf{A}_{i} \mathbf{Z}_{j}\right)\right\}$ e $\sigma^{2}=\left\{\sigma_{j}^{2}\right\}_{j=0}^{r}$. 
Igualando-se (12) a $s$, tem-se

$$
\mathbf{D} \hat{\sigma}^{2}=s
$$

Então, se D é não singular,

$$
\hat{\sigma}^{2}=\mathbf{D}^{-1} s
$$

é um estimador não-viesado de $\sigma^{2}$.

A matriz de dispersão de $\hat{\sigma}^{2}$ é

$$
\operatorname{var}\left(\hat{\sigma}^{2}\right)=\mathbf{D}^{-1} \operatorname{var}(s) \mathbf{D}^{-1,}
$$

onde os elementos de $\operatorname{var}(s)$ são variâncias e covariâncias das formas quadráticas usadas como elementos de $s$.

As soluções de (13) são chamadas as estimativas ANOVA para os componentes de variância, com dados desbalanceados.

Searle (1987) e Searle et al. (1992) apresentam esse método e discutem suas vantagens e desvantagens.

\subsubsection{Método I de Henderson}

Henderson (1953) descreve três métodos para estimar componentes de variância que são exatamente três diferentes maneiras de utilizar o método ANOVA de (13). Eles diferem somente nas diferentes formas quadráticas, nem sempre somas de quadrados, utilizadas no vetor $s$. Os métodos de Henderson podem produzir estimativas negativas.

No método I as formas quadráticas utilizadas são análogas às somas de quadrados usadas para dados balanceados, a analogia é tal que as somas de quadrados em dados balanceados tornam-se, para dados desbalanceados, em formas quadráticas que não são, necessariamente, somas de quadrados, pois nem sempre são não negativas, devido à estrutura desbalanceada dos dados. 
Assim, por exemplo, para o modelo

$$
\mathrm{y}_{\mathrm{ijk}}=\mu+\alpha_{\mathrm{i}}+\beta_{\mathrm{j}}+\gamma_{\mathrm{ij}}+\varepsilon_{\mathrm{ijk}}
$$

com $\mathrm{i}=1,2, \ldots, \mathrm{I}, \mathrm{j}=1,2, \ldots, \mathrm{J}$ e $\mathrm{k}=1,2, \ldots, \mathrm{n}$, as somas de quadrados

$$
\sum_{\mathrm{i}} \mathrm{J} n\left(\overline{\mathrm{y}}_{\mathrm{i}} \ldots-\overline{\mathrm{y}}_{\ldots} \ldots\right)^{2}=\sum_{\mathrm{i}} \mathrm{J} n \overline{\mathrm{y}}_{\mathrm{i}}^{2} \ldots-\mathrm{IJ} n \overline{\mathrm{y}}_{\ldots}^{2} .
$$

tornam-se, para dados desbalanceados, isto é, quando $\mathrm{k}=1,2, \ldots, \mathrm{n}_{\mathrm{ij}}$ :

$$
\sum_{i} n_{i}\left(\bar{y}_{i} \ldots-\bar{y}_{\ldots}\right)^{2}=\sum_{i} n_{i} \bar{y}_{i}^{2} \ldots-n_{\ldots} . \bar{y}_{\ldots}^{2}
$$

O método I utiliza o lado direito dessa equação.

A soma de quadrados para a interação, com dados balanceados é

$$
\sum_{\mathrm{i}} \sum_{j} \mathrm{n}\left(\overline{\mathrm{y}}_{\mathrm{i} \mathrm{j} .}-\overline{\mathrm{y}}_{\mathrm{i} . .}-\overline{\mathrm{y}}_{. \mathrm{j} .}+\overline{\mathrm{y}}_{\ldots} .\right)^{2}=\mathrm{n} \sum_{\mathrm{i}} \sum_{\mathrm{j}} \overline{\mathrm{y}}_{\mathrm{i} \mathrm{j} .}^{2}-\mathrm{J} \mathrm{n} \sum_{\mathrm{i}} \overline{\mathrm{y}}_{\mathrm{i} . .}^{2}-\operatorname{In} \sum_{j} \overline{\mathrm{y}}_{. \mathrm{j} .}^{2}+\mathrm{IJ} \mathrm{n} \overline{\mathrm{y}}_{\ldots .}^{2}
$$

A expressão, análoga ao lado direito, para dados desbalanceados, utilizada por esse método é:

$$
\sum_{i} \sum_{j} n_{i j} \bar{y}_{i j}^{2} .-\sum_{i} n_{i} \bar{y}_{i}^{2}-\sum_{j} n_{. j} \bar{y}_{. j}^{2}+n_{.} \bar{y}_{.}^{2} .
$$

que nem sempre é não negativa (Searle, 1971).

$\mathrm{O}$ método $\mathrm{I}$, consiste em igualar os quadrados médios às suas esperanças matemáticas e resolver o sistema de equações formado. Esse método fornece estimativas não-viesadas, com variância mínima, quando os dados são balanceados ou quando o modelo é aleatório e os efeitos não são correlacionados.

O método I de Henderson, não é adequado para modelos mistos. Pode ser adaptado a um modelo misto, alterando o modelo e tratando os efeitos fixos como não existentes ou como aleatórios, neste caso os estimadores dos componentes de variância para os verdadeiros efeitos aleatórios serão viesados. 


\subsubsection{Método II de Henderson}

O método II de Henderson, é uma extensão do método I, permitindo o seu uso em modelos mistos. $\mathrm{O}$ método consta de duas partes.

Primeiro faz a suposição temporária que os efeitos aleatórios são fixados, e para o modelo $\mathrm{y}=\mathrm{X} \theta+\mathbf{Z u}+e$ como definido em (2), resolvem-se as equações normais

$$
\left[\begin{array}{ll}
\mathbf{X}^{\prime} \mathbf{X} & \mathbf{X}^{\prime} \mathbf{Z} \\
\mathbf{Z}^{\prime} \mathbf{X} & \mathbf{Z}^{\prime} \mathbf{Z}
\end{array}\right]\left[\begin{array}{l}
\theta^{0} \\
\mathbf{u}^{0}
\end{array}\right]=\left[\begin{array}{l}
\mathbf{X}^{\prime} \mathbf{y} \\
\mathbf{Z}^{\prime} \mathbf{y}
\end{array}\right]
$$

para $\theta^{0}$. A seguir aplica-se o método I aos resíduos do modelo ajustado para os efeitos fixos.

$\mathrm{O}$ modelo $\mathrm{y}=\mathrm{X} \theta+\mathrm{Zu}+e$ definido em (2) pode ser reescrito como

$$
\mathrm{y}=\mu \mathbf{1}+\mathrm{X}^{*} \theta+\mathrm{Zu}+e
$$

Seja $\theta^{0}=\mathbf{L y}$, onde a matriz $\mathbf{L}$ é escolhida de modo que $\mathbf{y}^{*}=\mathbf{y}-\mathbf{X}^{*} \theta^{0}$ não dependa dos efeitos fixos (Searle 1968 e Searle et al. , 1992).

Assim, $\theta^{0}=$ Ly é tal que

$$
\mathrm{y}^{*}=\mu^{*} \mathbf{1}+\mathrm{Zu}+\mathrm{K}^{*} e
$$

onde $\mu^{*} \mathbf{1}=\mu\left(\mathbf{I}-\mathbf{X}^{*} \mathbf{L}\right) \mathbf{1}+\left(\mathbf{X}^{*}-\mathbf{X}^{*} \mathbf{L X}^{*}\right) \theta$ e $\mathbf{K}^{*}=\left(\mathbf{I}-\mathbf{X}^{*} \mathbf{L}\right)$

Logo, a equação do modelo para $\mathrm{y}^{*}$ tem os efeitos aleatórios da mesma forma que em y, ou seja $\mathrm{Zu}$. Então o método II consiste de aplicar o método I em y*.

Searle (1968) e Searle et al. (1992) mostram que se a matriz $\mathbf{L}$ satisfaz as condições:

(i) $\mathbf{X} * \mathbf{L Z}=\phi$;

(ii) todas as linhas de $\mathbf{X}^{*}-\mathbf{X}^{*} \mathbf{L} \mathbf{X}^{*}$ iguais;

(iii) todas as linhas de $\mathbf{X}^{*} \mathbf{L}$ têm a mesma soma.

então os resíduos do modelo ajustado para os efeitos fixos não dependem mais desses efeitos. 
Portanto, o método II de Henderson, consiste em estimar, em primeiro lugar, os efeitos fixos e, então aplicar o método I aos resíduos restantes. Para que os estimadores resultantes sejam não tendenciosos é necessário que os resíduos dependam apenas dos fatores aleatórios, a menos de uma constante que pode ser incluída no modelo. Há dois inconvenientes nesse método. Um deles, é o fato de não haver solução única e outro consiste em não se poder adotar modelos que incluam interações entre efeitos fixos e aleatórios, ou efeitos aleatórios aninhados dentro de efeitos fixos (Searle, 1968; Henderson, 1984).

\subsubsection{Método III de Henderson}

O método III de Henderson, também chamado método de ajuste de constantes, usa as reduções nas somas de quadrados do modelo completo e de sub-modelos para estimar os componentes de variância.

Para deduzir o método, considere o modelo misto definido em (2)

$$
\mathrm{y}=\mathbf{X} \theta+\mathbf{Z u}+e=\mathbf{W} \delta+e
$$

A matriz $\mathbf{W}$ pode ser particionada como $\left[\begin{array}{ll}\mathbf{W}_{1} & \mathbf{W}_{2}\end{array}\right]$, e $\delta$ ' pode ser particionada como $\left[\begin{array}{ll}\delta_{1} & \delta_{2}^{\prime}\end{array}\right]$ de acordo com $\mathbf{W}$. Assim o modelo pode ser reescrito como

$$
\mathrm{y}=\mathrm{W}_{1} \delta_{1}+\mathrm{W}_{2} \delta_{2}+e
$$

Note que nenhuma suposição é feita sobre a partição de $\mathbf{W}$ e $\delta$, no que se refere a efeitos fixos ou aleatórios.

Denotando $R\left(\delta_{1}, \delta_{2}\right)$ e $R\left(\delta_{1}\right)$, respectivamente, às reduções nas somas de quadrados do modelo completo e do sub-modelo $\mathrm{y}=\mathrm{W}_{1} \delta_{1}+e$, tem-se:

$$
\begin{array}{ll} 
& \mathrm{R}\left(\delta_{2} / \delta_{1}\right)=\mathrm{R}\left(\delta_{1}, \delta_{2}\right)-\mathrm{R}\left(\delta_{1}\right) \\
\text { e, portanto, } & \mathrm{E}\left[\mathrm{R}\left(\delta_{2} / \delta_{1}\right)\right]=\mathrm{E}\left[\mathrm{R}\left(\delta_{1}, \delta_{2}\right)\right]-\mathrm{E}\left[\mathrm{R}\left(\delta_{1}\right)\right]
\end{array}
$$


Mas

$$
\mathrm{R}\left(\delta_{1}, \delta_{2}\right)=\mathrm{y}^{\prime} \mathbf{W}\left(\mathbf{W}^{\prime} \mathbf{W}\right)^{g} \mathbf{W}^{\prime} \mathrm{y}
$$

e

$$
\mathrm{R}\left(\delta_{1}\right)=\mathrm{y}^{\prime} \mathbf{W}_{1}\left(\mathbf{W}_{1} \mathbf{W}_{1}\right)^{g} \mathbf{W}_{1}{ }^{\prime} \mathrm{y}
$$

Isto é, $\mathrm{R}\left(\delta_{1}, \delta_{2}\right)$ e $\mathrm{R}\left(\delta_{1}\right)$ são formas quadráticas de y, e tem-se:

$$
\begin{aligned}
& \mathrm{E}\left[\mathrm{R}\left(\delta_{1}, \delta_{2}\right)\right]=\mathrm{E}\left[\mathrm{y}^{\prime} \mathbf{W}\left(\mathbf{W}^{\prime} \mathbf{W}\right)^{g} \mathbf{W}^{\prime} \mathrm{y}\right] \\
& \mathrm{E}\left[\mathrm{R}\left(\delta_{1}, \delta_{2}\right)\right]=\operatorname{tr}\left[\mathbf{W}\left(\mathbf{W}^{\prime} \mathbf{W}\right)^{g} \mathbf{W}^{\prime} \operatorname{var}(\mathrm{y})\right]+\mathrm{E}\left(\mathrm{y}^{\prime}\right) \mathbf{W}\left(\mathbf{W}^{\prime} \mathbf{W}\right)^{g} \mathbf{W}^{\prime} \mathrm{E}(\mathrm{y})
\end{aligned}
$$

Mas

$$
\mathrm{E}(\mathrm{y})=\mathrm{E}(\mathbf{W} \delta+e)=\mathbf{W E}(\delta)
$$

e

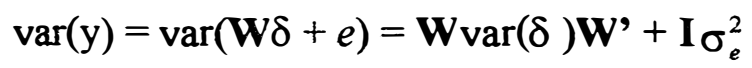

Logo

$$
\begin{aligned}
\mathrm{E}\left[\mathrm{R}\left(\delta_{1}, \delta_{2}\right)\right] & =\operatorname{tr}\left[\mathbf{W}\left(\mathrm{W}^{\prime} \mathbf{W}\right)^{g} \mathbf{W}^{\prime} \mathbf{W} \operatorname{var}(\delta) \mathbf{W}^{\prime}+\mathbf{W}\left(\mathbf{W}^{\prime} \mathbf{W}\right)^{g} \mathbf{W}^{\prime} \mathrm{I}_{\sigma_{e}^{2}}^{2}\right]+ \\
& +\mathrm{E}\left(\delta^{\prime}\right) \mathbf{W}^{\prime} \mathbf{W}\left(\mathbf{W}^{\prime} \mathbf{W}\right)^{g} \mathbf{W}^{\prime} \mathbf{W E}(\delta)= \\
& =\operatorname{tr}\left[\mathbf{W}^{\prime} \mathbf{W}^{\prime} \operatorname{var}(\delta)\right]+\sigma_{e}^{2} \operatorname{tr}\left[\mathbf{W}\left(\mathbf{W}^{\prime} \mathbf{W}\right)^{g} \mathbf{W}^{\prime}\right]+\mathrm{E}\left(\delta^{\prime}\right) \mathbf{W}^{\prime} \mathbf{W E}(\delta)= \\
& =\operatorname{tr}\left\{\mathbf{W}^{\prime} \mathbf{W}\left[\mathrm{E}\left(\delta \delta^{\prime}\right)-\mathrm{E}(\delta) \mathrm{E}\left(\delta^{\prime}\right)\right]\right\}+\sigma_{e}^{2} \operatorname{tr}\left[\mathbf{W}\left(\mathbf{W}^{\prime} \mathbf{W}\right)^{g} \mathbf{W}^{\prime}\right]+ \\
& +\operatorname{tr}\left\{\mathrm{E}\left(\delta^{\prime}\right) \mathbf{W}^{\prime} \mathbf{W E}(\delta)\right\}
\end{aligned}
$$

Portanto,

$$
\mathrm{E}\left[\mathrm{R}\left(\delta_{1}, \delta_{2}\right)\right]=\operatorname{tr}\left\{\mathbf{W}^{\prime} \mathbf{W E}\left(\delta \delta^{\prime}\right)\right\}+\sigma_{e}^{2} \operatorname{tr}\left[\mathbf{W}\left(\mathbf{W}^{\prime} \mathbf{W}\right)^{g} \mathbf{W}^{\prime}\right]
$$

ou

$$
\mathrm{E}\left[\mathrm{R}\left(\delta_{1}, \delta_{2}\right)\right]=\operatorname{tr}\left[\left(\begin{array}{ll}
\mathbf{W}_{1}^{\prime} \mathbf{W}_{1} & \mathbf{W}_{1}^{\prime} \mathbf{W}_{2} \\
\mathbf{W}_{2}^{\prime} \mathbf{W}_{1} & \mathbf{W}_{2}^{\prime} \mathbf{W}_{2}
\end{array}\right) \mathrm{E}\left(\delta \delta^{\prime}\right)\right]+\sigma_{e}^{2} \mathrm{r}(\mathbf{W})
$$

onde $r(\mathbf{W})$ é o posto da matriz $\mathbf{W}$. 
De modo análogo,

$$
\begin{aligned}
& \mathrm{E}\left[\mathrm{R}\left(\delta_{1}\right)\right]=\operatorname{tr}\left\{\mathbf{W}^{\prime} \mathbf{W}_{1}\left(\mathbf{W}_{1}^{\prime} \mathbf{W}_{1}\right)^{g} \mathbf{W}_{1}^{\prime} \mathbf{W E}\left(\delta \delta^{\prime}\right)\right\}+\sigma_{e}^{2} \operatorname{tr}\left[\mathbf{W}_{1}\left(\mathbf{W}_{1}^{\prime} \mathbf{W}_{1}\right)^{g} \mathbf{W}_{1}^{\prime}\right] \\
& \mathrm{E}\left[\mathrm{R}\left(\delta_{1}\right]=\operatorname{tr}\left[\left(\begin{array}{cc}
\mathbf{W}_{1}^{\prime} \mathbf{W}_{1} & \mathbf{W}_{1}^{\prime} \mathbf{W}_{2} \\
\mathbf{W}_{2}^{\prime} \mathbf{W}_{1} & \mathbf{W}_{2}^{\prime} \mathbf{W}_{1}\left(\mathbf{W}_{1}^{\prime} \mathbf{W}_{1}\right)^{g} \mathbf{W}_{1}^{\prime} \mathbf{W}_{2}
\end{array}\right) \mathrm{E}\left(\delta \delta^{\prime}\right)\right]+\sigma_{e}^{2} \mathrm{r}\left(\mathbf{W}_{1}\right)\right.
\end{aligned}
$$

Portanto $\mathrm{E}\left[\mathrm{R}\left(\delta_{2} / \delta_{1}\right)\right]=\mathrm{E}\left[\mathrm{R}\left(\delta_{1}, \delta_{2}\right)\right]-\mathrm{E}\left[\mathrm{R}\left(\delta_{1}\right)\right]$ é dador por:

$$
\mathrm{E}\left[\mathrm{R}\left(\delta_{2} / \delta_{1}\right)\right]=\operatorname{tr}\left[\left(\begin{array}{lc}
\phi & \phi \\
\phi & \mathbf{W}_{2}^{\prime}\left(\mathbf{I}-\mathbf{W}_{1}\left(\mathbf{W}_{1}^{\prime} \mathbf{W}_{1}\right)^{g} \mathbf{W}_{1}^{\prime}\right) \mathbf{W}_{2}
\end{array}\right) \mathrm{E}\left(\delta \delta^{\prime}\right)\right]+\sigma_{e}^{2}\left[\mathrm{r}(\mathbf{W})-\mathrm{r}\left(\mathbf{W}_{1}\right)\right]
$$

ou

$$
\mathrm{E}\left[\mathrm{R}\left(\delta_{2} / \delta_{1}\right)\right]=\operatorname{tr}\left\{\mathbf{W}_{2},\left(\mathbf{I}-\mathbf{W}_{1}\left(\mathbf{W}_{1}{ }^{\prime} \mathbf{W}_{1}\right)^{g} \mathbf{W}_{1}{ }^{\prime}\right) \mathbf{W}_{2} \mathrm{E}\left(\delta_{2} \delta_{2}{ }^{\prime}\right)\right\}+\sigma_{e}^{2}\left[\mathrm{r}(\mathbf{W})-\mathrm{r}\left(\mathbf{W}_{1}\right)\right]
$$

Por outro lado $\mathrm{E}\left(\delta_{2} \delta_{2}{ }^{\prime}\right)=\operatorname{var}\left(\delta_{2}\right)+\mathrm{E}\left(\delta_{2}\right) \mathrm{E}\left(\delta_{2}{ }^{\prime}\right)$ que não envolve $\delta_{1}$.

Note que $\mathrm{E}\left[\mathrm{R}\left(\delta_{2} / \delta_{1}\right)\right]$ não depende do vetor de efeitos $\delta_{1}$, sejam eles fixos ou aleatórios.

Assim, o método III de Henderson, cor siste em encontrar os estimadores para os componentes de variância, construindo um sistema de equações a partir das diferenças entre as reduções nas somas de quadrados do modelo completo e de sub-modelos, igualando-as às suas respectivas esperanças.

Para modelos mistos esse método é particularmente vantajoso, pois basta tomar o vetor $\delta_{1}$ como o vetor de efeitos fixos, e $\delta_{2}$ como o vetor de efeitos aleatórios, para a obtenção de estimadores de componentes de variância sem influências devidas aos efeitos fixos, pois $\mathrm{E}\left[\mathrm{R}\left(\delta_{2} / \delta_{1}\right)\right]$ não conterá termos devido a esses efeitos. Assim, a esperança é dada somente em função de $\sigma_{e}^{2}$ e das variâncias dos efeitos aleatórios em $\delta_{2}$, ou seja, os próprios componentes que se quer estimar.

O método III pode ser usado para modelos mistos e produz estimadores que são não-viesados. 


\subsubsection{Método da máxima verossimilhança : ML}

A estimação de componentes de variância por máxima verossimilhança foi iniciada com Fisher, em 1925. Uma das primeiras aplicações é vista em Hartley \& Rao (1967).

O método da máxima verossimilhança consiste na maximização da função densidade de probabilidade das observações, em relação aos efeitos fixos e aos componentes de variância.

Assim, o modelo misto (2), dado por

$$
\mathrm{y}=\mathbf{X} \theta+\mathbf{Z u}+e
$$

Assumindo que os efeitos aleatórios u e $e$ têm distribuição normal, com média zero, e matrizes de variâncias e covariâncias $\mathbf{G}$ e $\mathbf{I} \sigma_{e}^{2}$, respectivamente, o vetor y terá distribuição normal multivariada, com média $\mathbf{X} \theta$ e matriz de variâncias e covariâncias, $\mathbf{V}$ dada em (3), ou seja, $y \sim N(X \theta, V)$ com:

$$
\mathbf{V}=\mathbf{Z G Z}+\mathbf{I} \sigma_{e}^{2}
$$

A função de verossimilhança é:

$$
L=(2 \pi)^{-\frac{\mathbf{n}}{2}}|\mathbf{V}|^{-\frac{1}{2}} \exp \left[-\frac{1}{2}(\mathbf{y}-\mathbf{X} \theta)^{\prime} \mathbf{V}^{-1}(\mathbf{y}-\mathbf{X} \theta)\right]
$$

onde $|\mathbf{V}|$ é o determinante da matriz $\mathbf{V}$.

A função log verossimilhança é dada por:

$$
l=\log L=-\frac{\mathrm{n}}{2} \log (2 \pi)-\frac{1}{2} \log |\mathbf{V}|-\frac{1}{2}(\mathbf{y}-\mathbf{X} \theta)^{\prime} \mathbf{V}^{-1}(\mathbf{y}-\mathbf{X} \theta)
$$

Derivando $l$ em relação aos parâmetros desconhecidos, tem-se:

$$
\begin{aligned}
& \frac{\partial l}{\partial \theta}=\mathbf{X}^{\prime} \mathbf{V}^{-1} \mathrm{y}-\mathbf{X}^{\prime} \mathbf{V}^{-1} \mathbf{X} \theta \\
& \frac{\partial l}{\partial \sigma_{t}^{2}}=-\frac{1}{2} \frac{\partial}{\partial \sigma_{t}^{2}} \log |\mathbf{V}|-\frac{1}{2}(\mathrm{y}-\mathbf{X} \theta)^{\prime} \frac{\partial \mathbf{V}^{-1}}{\partial \sigma_{\imath}^{2}}(\mathrm{y}-\mathbf{X} \theta)
\end{aligned}
$$


Mas

$$
\frac{\partial}{\partial \sigma_{i}^{2}} \log |\mathbf{V}|=\operatorname{tr}\left(\mathbf{V}^{-1} \frac{\partial \mathbf{V}}{\partial \sigma_{i}^{2}}\right)=\operatorname{tr}\left(\mathbf{V}^{-1} \mathbf{Z}_{i} \mathbf{Z}_{i}{ }^{\prime}\right)
$$

e

$$
\frac{\partial \mathbf{V}^{-1}}{\partial \sigma_{1}^{2}}=-\mathbf{V}^{-1} \frac{\partial \mathbf{V}}{\partial \sigma_{1}^{2}}-\mathbf{V}^{-1}=-\mathbf{V}^{-1} \mathbf{Z}_{1} \mathbf{Z}_{\imath}^{\prime} \mathbf{V}^{-1}
$$

Logo,

$$
\frac{\partial l}{\partial \sigma_{i}^{2}}=-\frac{1}{2} \operatorname{tr}\left(\mathbf{V}^{-1} \mathbf{Z}_{i} \mathbf{Z}_{i}{ }^{\prime}\right)+\frac{1}{2}(\mathrm{y}-\mathbf{X} \theta)^{\prime} \mathbf{V}^{-1} \mathbf{Z}_{i} \mathbf{Z}_{i}^{\prime} \mathbf{V}^{-1}(\mathrm{y}-\mathbf{X} \theta)
$$

Igualando-se as derivadas parciais (15) e (16) a zero, tem-se:

$$
\begin{aligned}
& \mathbf{X}^{\prime} \widetilde{\mathbf{V}}^{-1} \mathbf{X} \tilde{\theta}=\mathbf{X}^{\prime} \tilde{\mathbf{V}}^{-1} \mathbf{y} \\
& \operatorname{tr}\left(\tilde{\mathbf{V}}^{-1} \mathbf{Z}_{i} \mathbf{Z}_{i}{ }^{\prime}\right)=(\mathbf{y}-\mathbf{X} \tilde{\theta})^{\prime} \widetilde{\mathbf{V}}^{-1} \mathbf{Z}_{i} \mathbf{Z}_{i}{ }^{\cdot} \widetilde{\mathbf{V}}^{-1}(\mathbf{y}-\mathbf{X} \widetilde{\theta})
\end{aligned}
$$

onde $\widetilde{\theta}$ e $\widetilde{\mathbf{V}}$ representando as estimativas ML $\operatorname{de} \theta$ e V.

Uma expressão mais simples para (18) pode ser deduzida considerando-se

$$
\mathbf{P}=\mathbf{V}^{-1}-\mathbf{V}^{-1} \mathbf{X}\left(\mathbf{X}^{\prime} \mathbf{V}^{-1} \mathbf{X}\right)^{g} \mathbf{X}^{\prime} \mathbf{V}^{-1}
$$

Então

$$
\mathbf{P y}=\mathbf{V}^{-1}(\mathrm{y}-\mathbf{X} \theta)
$$

Assim,

$$
\widetilde{\mathbf{P}} \mathrm{y}=\widetilde{\mathbf{V}}^{-1}(\mathrm{y}-\mathbf{X} \tilde{\theta})
$$

em que define-se $\widetilde{\mathbf{P}}$ de $\mathbf{P}$, substituindo-se $\mathbf{V}$ por $\widetilde{\mathbf{V}}$.

Logo, as equações de ML (17) e (18) são:

$$
\mathbf{X}^{\cdot} \widetilde{\mathbf{V}}^{-1} \mathbf{X} \tilde{\theta}=\mathbf{X}^{\prime} \widetilde{\mathbf{V}}^{-1} \mathbf{y}
$$

e

$$
\left\{\operatorname{tr}\left(\widetilde{\mathbf{V}}^{-1} \mathbf{Z}_{i} \mathbf{Z}_{i}{ }^{\prime}\right)\right\}=\left\{\mathbf{y}^{\top} \widetilde{\mathbf{P}} \mathbf{Z}_{i} \mathbf{Z}_{i}{ }^{\prime} \widetilde{\mathbf{P}}_{\mathbf{y}}\right\}
$$

O PROC MIXED implementa o método ML construindo uma função objetivo para ML, dada por $-2 l$, ou seja, $-2 \log L$. 
Assim, menos duas vezes a função log verossimilhança (14) é dada por:

$$
-2 l=-2 \log L=\log |\mathbf{V}|+(\mathbf{y}-\mathbf{X} \theta)^{\prime} \mathbf{V}^{-1}(\mathbf{y}-\mathbf{X} \theta)+n \log (2 \pi)
$$

Essa equação por ser reescrita segundo Wolfinger et al. (1994), por:

$$
-2 \log L=\log |\mathbf{V}|+\mathbf{n} \log \mathbf{r}^{\prime} \mathbf{V}^{-1} \mathbf{r}+\mathrm{n}[1+\log (2 \pi / \mathrm{n})]
$$

onde $\mathbf{r}=\mathrm{y}-\mathbf{X}\left(\mathbf{X}^{\top} \mathbf{V}^{-1} \mathbf{X}\right)^{g} \mathbf{X}^{\top} \mathbf{V}^{-1} \mathbf{y}$.

Essa expressão é a função objetivo para ML utilizada pelo PROC MIXED do sistema estatístico SAS (Littell et al., 1996; SAS, 1996; 1997).

Essas equações são não lineares e são resolvidas numericamente, em geral por processos iterativos. O processo é repetido até que o critério de convergência adotado, seja satisfeito. Assim, o método da máxima verossimilhança é iterativo e fornece sempre estimativas não-negativas de componentes de variância. No entanto, tais estimativas são viesadas pois o método não considera a perda de graus de liberdade, resultante da estimação dos efeitos fixos do modelo.

\subsubsection{Método da máxima verossimilhança restrita : REML}

Esse procedimento é uma variante do método de máxima verossimilhança, para modelos mistos e foi proposto por Patterson \& Thompson (1971) para delineamentos em blocos casualizados.

Os estimadores REML são obtidos maximizando-se a parte da função de verossimilhança que é invariante ao parâmetro de locação; isto é, em termos do modelo misto $\mathrm{y}=\mathbf{X} \theta+\mathbf{Z u}+e$, invariante a $\mathbf{X} \theta$. Ou de outra maneira, os estimadores REML maximizam a função de verossimilhança de um vetor de combinações lineares das observações que são invariantes a $\mathbf{X} \theta$. 
Seja K'y o vetor de combinações lineares. Então $\mathbf{K}^{\prime} \mathbf{y}=\mathbf{K}^{\prime} \mathbf{X} \theta+\mathbf{K}^{\prime} \mathbf{Z} \mathbf{u}+\mathbf{K}^{\prime} \mathbf{e}$ é invariante a $\mathbf{X} \theta$ se e somente se $\mathbf{K}^{\prime} \mathbf{X}=\phi . \quad$ Mas $\mathbf{K}^{\prime} \mathbf{X}=\phi$ se $\mathbf{K}^{\prime}=\mathbf{T M}$ para $\mathbf{M}=\mathbf{I}-\mathbf{X}\left(\mathbf{X}^{\prime} \mathbf{X}\right)^{g} \mathbf{X}^{\prime}$, para algum $\mathbf{T}$ onde $\mathbf{M}$ e $\mathbf{T}$ têm posto linha completo.

Com $y \sim N(\mathbf{X} \theta, \mathbf{V})$, tem-se que para $\mathbf{K}^{\prime} \mathbf{X}=\phi$,

$$
\mathbf{K}^{\prime} \mathbf{y} \sim \mathrm{N}\left(\boldsymbol{\phi}, \mathbf{K}^{\prime} \mathbf{V K}\right)
$$

As equações REML podem ser deduzidas das equações ML de (21), ou seja de

$$
\left\{\operatorname{tr}\left(\tilde{\mathbf{V}}^{-1} \mathbf{Z}_{i} \mathbf{Z}_{i}{ }^{\prime}\right)\right\}=\left\{\mathbf{y}^{\prime} \widetilde{\mathbf{P}} \mathbf{Z}_{i} \mathbf{Z}_{i}{ }^{\prime} \widetilde{\mathbf{P}} \mathbf{y}\right\}
$$

Substituindo-se:

y por $\mathbf{K}^{\prime} \mathbf{y}, \quad \mathbf{X}$ por $\mathbf{K}^{\prime} \mathbf{X}=\phi, \mathbf{Z}$ por $\mathbf{K}^{\prime} \mathbf{Z}$ e $\mathbf{V}$ por $\mathbf{K}^{\prime} \mathbf{V K}$ Assim, (21) pode ser reescrita como

$$
\left\{\operatorname{tr}\left[\left(K^{\prime} \tilde{\mathbf{V}} K\right)^{-1} K^{\prime} \mathbf{Z}_{i} \mathbf{Z}_{i}{ }^{\prime} K\right]\right\}=\left\{\mathbf{y}^{\prime} \mathbf{K}\left(K^{\prime} \tilde{\mathbf{V}} K\right)^{-1} K^{\prime} \mathbf{Z}_{i} \mathbf{Z}_{i}{ }^{\prime} \mathbf{K}\left(\mathbf{K}^{\prime} \tilde{\mathbf{V}} \mathbf{K}\right)^{-1} K^{\prime} \mathbf{y}\right\}
$$

Mas, por (19)

$$
\mathbf{P}=\mathbf{V}^{-1}-\mathbf{V}^{-1} \mathbf{X}\left(\mathbf{X}^{\cdot} \mathbf{V}^{-1} \mathbf{X}\right)^{g} \mathbf{X}^{\prime} \mathbf{V}^{-1}=\mathbf{K}\left(K^{\prime} \mathbf{V K}\right)^{-1} K^{\prime}
$$

Logo, (23) pode ser reescrita como:

$$
\left\{\operatorname{tr}\left(\widetilde{\mathbf{P}} \mathbf{Z}_{i} \mathbf{Z}_{i}{ }^{\prime}\right\}=\left\{\mathrm{y}^{\prime} \widetilde{\mathbf{P}} \mathbf{Z}_{i} \mathbf{Z}_{i}{ }^{\prime} \widetilde{\mathbf{P}} \mathbf{y}\right\}\right.
$$

Essas são as equações REML. Comparando-as com as equações ML de (21) observa-se que elas são iguais exceto pelo fato de que $\mathbf{V}^{-1}$ nas equações $M L$ é substituído por $\mathbf{P}$ nas equações REML.

As equações REML também podem ser deduzidas das equações ML substituindose:

y por $\mathbf{K}^{\prime} \mathbf{y}, \quad \mathbf{X}$ por $\mathbf{K}^{\prime} \mathbf{X}=\phi, \mathbf{Z}$ por $\mathbf{K}^{\prime} \mathbf{Z}$ e $\mathbf{V}$ por $\mathbf{K}^{\prime} \mathbf{V K}$

O procedimento MIXED do SAS implementa o método REML construindo a função $-2 \log L_{\mathrm{R}}$.

Assim, para a estimação REML, -2 log verossimilhança restrita é dada segundo Verneque (1994), por:

$$
-2 \log L_{R}=\log |\mathbf{V}|+(\mathbf{y}-\mathbf{X} \theta)^{\prime} \mathbf{V}^{-1}(\mathbf{y}-\mathbf{X} \theta)+\log \left|\mathbf{X}^{\prime} \mathbf{V}^{-1} \mathbf{X}\right|+(\mathrm{n}-\mathrm{k}) \log (2 \pi)
$$


onde $\mathrm{k}$ é o rank da matriz $\mathbf{X}$.

Essa equação por ser reescrita segundo Wolfinger et al. (1994), por:

$$
-2 \log L_{\mathrm{R}}=\log |\mathbf{V}|+\log \left|\mathbf{X}^{\prime} \mathbf{V}^{-1} \mathbf{X}\right|+(\mathrm{n}-\mathrm{k}) \log \mathbf{r}^{\prime} \mathbf{V}^{-1} \mathbf{r}+(\mathrm{n}-\mathrm{k})\{1+\log [2 \pi /(\mathrm{n}-\mathrm{k})]\}
$$

onde $\mathbf{r}=\mathrm{y}-\mathbf{X}\left(\mathbf{X}^{\top} \mathbf{V}^{-1} \mathbf{X}\right)^{g} \mathbf{X}^{9} \mathbf{V}^{-1} \mathrm{y}$ e k é o rank da matriz $\mathbf{X}$.

O PROC MIXED minimiza essa função (25) utilizando uma modificação do método iterativo de Newton-Raphson para obter as estimativas REML (Littell et al., 1996; SAS, 1996; 1997).

No método da máxima verossimilhança restrita, cada observação é dividida em duas partes independentes, uma referente aos efeitos fixos e outra aos aleatórios, de maneira que a função densidade de probabilidade das observações é dada pela soma das funções densidade de probabilidade de cada parte. A maximização da função densidade de probabilidade da parte referente aos efeitos aleatórios, em relação aos componentes de variância, elimina o viés resultante da perda de graus de liberdade na estimação dos efeitos fixos do modelo, observada em 4.2.2.5.

O método REML tem sido considerado (Searle, 1971; Corbeil \& Searle, 1976; Harville, 1977; Henderson, 1984; Searle et al., 1992, entre outros) o preferido para estimar componentes de variância de dados desbalanceados. As razões para essa preferência são justificadas pelas propriedades desses estimadores.

Algumas dessas propriedades são:

- Os estimadores são funções de estatísticas suficientes, consistentes e assintoticamente normais e eficientes;

- Os estimadores REML com dados balanceados são idênticas aos estimadores ANOVA, são de variância mínima e não-viesados.

- Os estimadores REML levam em conta os graus de liberdade envolvidos nas estimativas dos efeitos fixos, ao passo que os estimadores ML não.

Por tais propriedades, os estimadores REML tem sido tomados como default de vários sistemas estatísticos, entre eles o PROC MIXED do sistema estatístico SAS. Que será apresentado no item 4 deste estudo. 
Há, no entanto, alguns fatos que devem ser considerados:

- No caso de dados desbalanceados os estimadores ML e os estimadores REML são, ambos, viesados.

- Os estimadores ML e REML, dos componentes de variância, não são formas explícitas, isto é, o estimador de cada componente está em função dos estimadores dos outros componentes, e só podem ser obtidos por meio de métodos numéricos iterativos.

\subsubsection{Método de estimação quadrática não-viesada de norma mínima : MINQUE}

Rao $(1970 ; 1971 a ; 1972)$ descreve um método de estimação que é derivado de modo que o estimador minimize a norma euclidiana da matriz núcleo, que seja uma forma quadrática das observações e que seja não-viesado. Seu desen olvimento envolve álgebra extensiva e seu conceito utiliza valores escolhidos, à priori, para os componentes de variância desconhecidos.

A estimação dos componentes de variância pelo método MINQUE, é feita com base na equação MINQUE, a seguir:

$$
\left\{\operatorname{tr}\left(\mathbf{P}_{\mathbf{w}} \mathbf{V}_{\mathbf{i}} \mathbf{P}_{\mathbf{w}} \mathbf{V}_{\mathbf{j}}\right)\right\} \hat{s}=\left\{\mathbf{y}^{\prime} \mathbf{P}_{\mathbf{w}} \mathbf{V}_{\mathbf{i}} \mathbf{P}_{\mathbf{w}} \mathbf{y}\right\}
$$

onde $\hat{s}$ é o vetor de soluções dos componentes de variância,

$\mathbf{P}_{w}=\mathbf{V}_{w}^{-1}-\mathbf{V}_{w}^{-1} \mathbf{X}\left(\mathbf{X}^{\prime} \mathbf{V}_{w}^{-1} \mathbf{X}\right)^{8} \mathbf{X}^{\prime} \mathbf{V}_{w}^{-1}$ como em (19) com $\mathbf{V}_{\mathbf{w}}$ em lugar de $\mathbf{V}$

$\mathbf{V}_{\mathbf{w}}$ é uma estimativa à priori da matriz de variâncias e covariâncias

Esse método tem duas vantagens: não envolve suposições de normalidade como o ML e REML, e as equações MINQUE têm soluções explícitas.

Por outro lado, a solução depende do conhecimento à priori dos valores dos componentes de variância a serem estimados, ou seja, depende de valores estimados à priori, usados em $\mathbf{V}_{\mathbf{w}}$. Assim, diferentes valores de $\mathbf{V}_{\mathbf{w}}$ podem levar a diferentes 
estimativas para um mesmo conjunto de dados. Obtém-se portanto "um" estimador MINQUE e não "o" estimador MINQUE.

Um relacionamento, importante, que existe entre REML e MINQUE é que se o valor inicial no processo iterativo REML é $\mathbf{V}_{\mathbf{w}}$, então a primeira solução é uma estimativa MINQUE. Nesse caso pode-se escrever, como sugere Searle et al. (1992):

Uma solução MINQUE = primeira iteração de REML

O estimador MINQUE utiliza valores estimados à priori em $\mathbf{V}_{\mathbf{w}}$, ou seja uma estimativa à priori para $\mathbf{V}$, matriz de variâncias e covariâncias. Nenhuma iteração está envolvida. No entanto, obtida uma solução, por exemplo $\widetilde{\mathbf{V}}_{1}$, existe a idéia de usá-la como uma nova estimativa em $\mathbf{V}_{\mathbf{w}}$, a partir da qual um novo conjunto de equações pode ser estabelecido e resolvido, produzindo $\widetilde{\mathbf{V}}_{2}$ e assim sucessivamente. Isto leva a usar as equações MINQUE iterativamente, caracterizando o método do MINQUE Iterativo, e os estimadores I-MINQUE. Portanto

$$
\text { I-MINQUE }=\text { REML }
$$

Além disso, Brown (1976) mostra que mesmo sem suposições de normalidade sobre $\mathrm{y}$, as soluções I-MINQUE têm propriodades de normalidade para grandes amostras.

\subsubsection{Método de estimação quadrática não-viesada de variância mínima : MIVQUE A MIVQUEO}

Como visto em 4.2.2.7, o método MINQUE não exige qualquer suposição sobre a forma da distribuição da variável aleatória y. No entanto, se a suposição usual de normalidade é satisfeita, o estimador MINQUE tem a propriedade de ser uma forma 
quadrática não-viesada das observações com variância mínima, ou seja é um estimador quadrático não-viesado de variância mínima, conhecido como MIVQUE. (Searle, 1987).

Sob normalidade, os estimadores, MINQUE e MIVQUE, são equivalentes, e podem ser obtidos pela expressão (26). Assim, o método MIVQUE é o mais comumente utilizado e conhecido, tendo em vista que na maioria das aplicações práticas, a normalidade é assumida.

Swallow \& Monahan (1984) descrevem o procedimento MIVQUE em concordância com os valores estimados à priori em $\mathbf{V}_{\mathbf{w}}$, MIVQUE(A) e MIVQUE0.

O estimador MIVQUE(A) usa a equação (26), tomando as estimativas ANOVA como valores à priori. Embora a teoria sobre MIVQUE especifique, que os valores à priori devam ser independentes dos dados, a literatura consultada justifica o uso das estimativas ANOVA em decorrência da facilidade de obtenção.

O estimador MIVQUE0 é o MIVQUE com a suposição à priori de que a matriz de variâncias e covariâncias é a matriz identidade.

Assim, o estimador MIVQUE0 utiliza a equação (26) considerando $\mathbf{V}_{\mathbf{w}}=\mathbf{I}$. Logo,

$$
\left\{\operatorname{tr}\left(\mathbf{P V} \mathbf{i}_{\mathbf{i}} \mathbf{P V}\right)\right\} \hat{s}=\left\{\mathbf{y}^{\prime} \mathbf{P} \mathbf{V}_{\mathbf{i}} \mathbf{P y}\right\}
$$

onde $\hat{s}$ é o vetor de soluções dos componentes de variância e $\mathbf{P}=\mathbf{I}-\mathbf{X}\left(\mathbf{X}^{\prime} \mathbf{X}\right)^{g} \mathbf{X}^{\prime}$ conforme visto em (19) com $\mathbf{V}=\mathbf{I}$.

Como será visto no item 4.3.2, o método MIVQUE0 é o método default do SAS no procedimento VARCOMP e um dos métodos disponíveis no procedimento MIXED (SAS, 1990; 1997). 


\subsubsection{Estimação dos efeitos fixos}

$\mathrm{Na}$ análise do modelo linear misto tem-se, também, interesse na estimação e testes de hipóteses dos efeitos fixos. Entretanto, para a estimativa de uma função estimável dos parâmetros de efeitos fixos, no modelo misto, é necessário o conhecimento das estimativas dos componentes de variância. Assim as estimativas dos parâmetros de efeitos fixos, no modelo misto, dependem diretamente dos métodos utilizados na obtenção das estimativas dos componentes de variância.

A estimação pode ser mais dificil no modelo misto do que no modelo de efeitos fixos. Não se tem apenas $\theta$ como no modelo de efeitos fixos, mas também parâmetros de efeitos aleatórios desconhecidos em u, G e R. O método dos mínimos quadrados ordinários que minimiza $(\mathrm{y}-\mathrm{X} \theta)^{\prime}(\mathrm{y}-\mathrm{X} \theta)$ com respeito à $\theta$, como visto em 4.1.1 é adequado ao modelo linear ordinário de Gass-Markov, que, como descrito em (1) pressupõe $\mathrm{V}(e)=\mathbf{I} \sigma^{2}$ Tal método fornece os estimadores do tipo OLSE (Ordinary Least Squares Estimator), e fornece a aproximação de mínimos quadrados para $\mathrm{y}=\mathrm{X} \theta$, dada por $\operatorname{OLSE}(\mathrm{X} \theta)=\hat{\mathrm{y}}=\mathbf{X}\left(\mathbf{X}^{\prime} \mathbf{X}\right)^{g} \mathbf{X}^{\prime} \mathbf{y}=\mathbf{X} \theta^{0}$, sem ser influenciado pela matriz de

variâncias e covariâncias $\left(V=I \sigma^{2}\right)$ e, desse modo, para os modelos mistos eles nem sempre são estimadores do tipo BLUE.

Um estimador mais realista pode ser obtido por meio do método dos mínimos quadrados generalizados, GLSE (Generalized Least Squares Estimator), que considera uma matriz $\mathbf{V}$ mais geral e que minimiza $(y-X \theta)^{\prime} V^{-1}(y-X \theta)$, fornecendo o sistema de equações normais generalizadas $X^{\prime} V^{-1} X \theta=X^{\prime} V^{-1} y$.

Para obter-se o BLUE de uma função estimável $k^{\prime} \theta$, basta utilizar-se o teorema de Gauss-Markov: BLUE de $k^{\prime} \theta=k^{\prime} \theta^{0}$ onde $\theta^{0}$ é qualquer solução das equações normais generalizadas.

Assim, para o modelo (2) tem-se:

$$
\operatorname{BLUE}\left(\lambda^{\prime} \mathbf{X} \theta\right)=\lambda^{\prime} \mathbf{X}\left(\mathbf{X}^{\prime} \mathbf{V}^{-1} \mathbf{X}\right)^{g} \mathbf{X}^{\prime} \mathbf{V}^{-1} \mathbf{y}
$$


Desse modo, os componentes de variância são considerados nas estimativas das funções estimáveis $\lambda^{\prime} \mathbf{X} \theta$ dos efeitos fixos.

A variância de (28) é:

$$
\operatorname{var}\left[\operatorname{BLUE}\left(\lambda^{\prime} \mathbf{X} \theta\right)\right]=\lambda^{\prime} \mathbf{X}\left(\mathbf{X}^{\prime} \mathbf{V}^{-1} \mathbf{X}\right)^{\mathbf{g}} \mathbf{X}^{\prime} \lambda
$$

Esse método de estimação deve ser preferido sobre o método de mínimos quadrados ordinários: $\mathbf{X}^{\prime} \mathbf{X} \theta^{0}{ }_{\text {OLSE }}=\mathbf{X}^{\prime} \mathbf{y}$, quando os modelos mistos estão em estudo.

Uma dificuldade com (28) e (29) é que elas requerem que $\mathbf{V}=\operatorname{var}(\mathrm{y})$ seja conhecida, ou seja, envolvem os componentes de variância. Em muitas situações práticas os componentes de variância não são conhecidos. Nesses casos, uma estratégia interessante consiste na obtenção de um conjunto de estimativas dos componentes de variância, que serão utilizadas em lugar dos componentes em V (Kackar \& Harville, 1981). Então, substitui-se $\mathbf{V}$ por $\hat{\mathbf{V}}$ e assim tem-se

$$
\operatorname{BLUE}_{\hat{\mathbf{v}}}\left(\lambda^{\prime} \mathbf{X} \theta\right)=\lambda^{\prime} \mathbf{X}\left(\mathbf{X}^{\prime} \hat{\mathbf{V}}^{-1} \mathbf{X}\right)^{g} \mathbf{X}^{\prime} \hat{\mathbf{V}}^{-1} \mathbf{y}
$$

Segundo Milliken \& Johnson (1984) e Kackar \& Harville (1984), para dados desbalanceados, o estimador de mínimos quadrados generalizados de uma função estimável $\lambda^{\prime} \mathbf{X} \theta$ é $\lambda^{\prime} \mathbf{X} \hat{\theta}_{\hat{\mathrm{v}}}$ onde

$$
\lambda^{\prime} \mathbf{X} \hat{\theta}_{\hat{\mathbf{v}}}=\lambda{ }^{\prime} \mathbf{X}\left(\mathbf{X}^{\prime} \hat{\mathbf{v}}^{-1} \mathbf{X}\right)^{g} \mathbf{X}^{\prime} \hat{\mathbf{v}}^{-1} \mathbf{y}
$$

e a estimativa da variância de $\lambda^{\top} \mathbf{X} \hat{\theta}_{\hat{\mathrm{V}}}$ é aproximadamente dada por

$$
\operatorname{var}\left[\lambda^{\prime} \mathbf{X} \hat{\theta}_{\hat{\mathrm{v}}}\right]=\lambda^{\prime} \mathbf{X}\left(\mathbf{X}^{\prime} \hat{\mathrm{V}}^{-1} \mathbf{X}\right) \mathbf{X}^{\prime} \lambda
$$

Ademais, assintoticamente

$$
\frac{\lambda^{\prime} \mathbf{X} \hat{\theta}_{\hat{\mathbf{V}}}-\lambda^{\prime} \mathbf{X} \theta}{\sqrt{\lambda^{\prime} \mathbf{X}\left(\mathbf{X}^{\prime} \hat{\mathbf{V}}^{-1} \mathbf{X}\right) \mathbf{X}^{\prime} \lambda}} \sim N(0,1)
$$

Assim, a estimação por intervalo é feita através de

$$
\operatorname{IC}\left[\lambda^{\prime} \theta\right]_{1-\alpha}: \quad \lambda^{\prime} \mathbf{X} \hat{\theta}_{\hat{\mathrm{V}}} \pm z_{\alpha / 2} \sqrt{\lambda^{\prime} \mathbf{X}\left(\mathbf{X}^{\prime} \hat{\mathbf{V}}^{-1} \mathbf{X}\right) \mathbf{X}^{\prime} \lambda}
$$

onde $z_{\alpha / 2}$ é o percentil $100(1-\alpha / 2)$ da distribuição normal padrão, $N(0,1)$. 


\subsubsection{Testes de hipóteses}

Para inferências relativas aos parâmetros de efeitos fixos e aleatórios no modelo misto, consideram-se combinações lineares de funções estimáveis da forma $\mathbf{L}\left[\begin{array}{l}\theta \\ \mathbf{u}\end{array}\right]$. Funções dessa natureza são ditas estimáveis, se a parte fixa $\theta$ satisfaz a exigência de estimabilidade (Searle, 1971). Quando o interesse consiste apenas em inferências sobre funções estimáveis dos efeitos fixos, cada componente corresponde a parte u em L deve ser igualado a zero.

As inferências estatísticas podem ser obtidas para testar as hipóteses

$$
\mathrm{H}_{0}: \mathbf{L}\left[\begin{array}{l}
\theta \\
\mathrm{u}
\end{array}\right]=\phi
$$

e para a construção de intervalos de confiança.

Quando $\mathbf{L}$ consiste de apenas uma linha, uma estatística t pode ser construída como

$$
t=\frac{L\left[\begin{array}{l}
\hat{\theta} \\
\hat{u}
\end{array}\right]}{\sqrt{\mathbf{L} \hat{C} L^{\prime}}}
$$

onde

$$
\hat{\mathbf{C}}=\left[\begin{array}{cc}
\mathbf{X}^{\prime} \mathbf{X} & \mathbf{X}^{\prime} \mathbf{Z} \\
\mathbf{Z}^{\prime} \mathbf{X} & \mathbf{Z} \mathbf{Z}+\hat{\sigma}_{e}^{2} \hat{\mathbf{G}}^{-1}
\end{array}\right]^{g}
$$

ou seja, $\hat{\mathbf{C}}$ é uma matriz inversa generalizada dos coeficientes das "equações do modelo misto" (7), conforme já visto em (11).

Sob a suposição de normalidade de u e de $e$, em geral $\mathrm{t}$ tem uma distribuição aproximada " $t$ ", e seus graus de liberdade podem ser determinados pela fórmula de Satterthwaite. 
Seja d o número de graus de liberdade aproximado, então o intervalo de confiança é

$$
\mathbf{L}\left[\begin{array}{c}
\hat{\theta} \\
\hat{\mathrm{u}}
\end{array}\right] \pm \mathrm{t}_{\mathrm{d}, \alpha / 2} \sqrt{\mathbf{L} \hat{\mathbf{C}} \mathbf{L}}
$$

onde $t_{d \alpha / 2}$ é o percentil $100(1-\alpha / 2)$ da distribuição $t_{d}$.

Quando o número de linhas de $\mathbf{L}$ é maior do que 1 , considera-se a estatística $\mathrm{F}$ dada por:

$$
F=\frac{\left[\begin{array}{l}
\hat{\theta} \\
\hat{u}
\end{array}\right]^{\prime} \mathbf{L}^{\prime}\left(\mathbf{L} \hat{\mathbf{C}} \mathbf{L}^{\prime}\right)^{-1} \mathbf{L}\left[\begin{array}{l}
\hat{\theta} \\
\hat{\mathbf{u}}
\end{array}\right]}{\operatorname{rank}(\mathbf{L})}
$$

Analogamente a t, F tem uma distribuição aproximada "F" com graus de liberdade do numerador igual a $r(\mathbf{L})$ e graus de liberdade do denominador igual a $\mathrm{d}$.

Por default o PROC MIXED executa os testes de hipóteses sobre os efeitos fixos construindo a matriz $\mathbf{L}$ com coeficientes de funções estimáveis do tipo III para cada efeito. A matriz $\mathbf{L}$ é utilizada para calcular a seguinte estatística $\mathrm{F}$ :

$$
\mathrm{F}=\frac{(\mathbf{L} \hat{\theta})^{\prime}\left[\mathbf{L}\left(\mathbf{X}^{\prime} \hat{\mathbf{V}}^{-1} \mathbf{X}\right)^{g} \mathbf{L}^{\prime}\right]^{-1} \mathbf{L} \hat{\theta}}{\operatorname{rank}(\mathbf{L})}
$$

A estatística F tem uma distribuição aproximada "F" com graus de liberdade do numerador igual a $\mathrm{r}(\mathbf{L})$ e graus de liberdade do denominador igual a $\mathrm{d}$.

\subsubsection{Estruturas gerais de covariâncias}

Conforme visto em 4.2, a análise de modelos mistos envolvem duas partes: a análise da parte fixa e a análise da parte aleatória. Tanto a estimação dos efeitos fixos 
quanto a predição dos efeitos aleatórios dependem da estimação dos componentes de variância.

A estimação dos componentes de variância dependem da estrutura da matriz de covariâncias dos efeitos aleatórios $\mathrm{G}$, de $\mathbf{R}=\mathbf{I} \sigma_{e}^{2}$ e dos método de estimação utilizados como já dito em 4.2.2.

Várias estruturas de covariâncias podem ser especificas segundo as características dos dados, para a matriz G. (Searle et al., 1992; Wolfinger, 1993; Latour et al., 1994; Littell et al., 1996, entre outros).

Assim, neste estudo, visando ilustrar os métodos de estimação e viabilizar uma comparação entre eles optou-se por adotar $\mathbf{R}=\mathbf{I} \sigma_{e}^{2}$ e as seguintes estruturas de $G$, disponíveis no PROC MIXED.

- diagonal

- componentes de variância

- simetria composta (diagonal mais covariância comum)

- Huynh-Feldt (Huynh \& Feldt, 1970)

Essas estruturas foram escolhidas pois estão entre as mais comumente utilizadas.

O procedimento MIXED do SAS, oferece várias opções para a estrutura de covariâncias e essas podem ser estimadas por meio dos métodos MIVQUE0, ML e REML.

Assim, neste trabalho serão consideradas as seguintes estruturas de G:

- Componentes de variância cujo elemento $(i, j)$ é dado por $\sigma_{k}^{2} 1(i=j)$ onde $\mathrm{i}$ corresponde ao $k$-ésimo elemento e $1(\mathrm{i}=\mathrm{j})$ é igual a 1 quando $\mathrm{i}=\mathrm{j}$, e zero caso contrário. VC é a estrutura default para G.

- Diagonal dada por $\mathbf{I} \sigma^{2}$.

- Simetria composta cujo elemento $(i, j)$ é $\sigma_{1}+\sigma^{2} 1(i=j)$

- Huynh-Feldt onde os elementos $(i, j)$ são da forma $\left(\sigma_{i}^{2}+\sigma_{j}^{2}\right) / 2-\lambda 1(i \neq j)$ onde $\lambda$ são autovalores positivos associados à matriz de covariâncias e $1(i \neq j)$ é igual a 1 quando $\mathrm{i} \neq \mathrm{j}$, e zero caso contrário 
O número de parâmetros de covariâncias nessas estruturas é igual ao número de efeitos aleatórios, 1,2 e $\mathrm{t}+1$, respectivamente, onde $\mathrm{t}$ é igual a dimensão da matriz de covariâncias.

Na Tabela 2 tem-se a notação e exemplos para essas estruturas de G.

Supondo $\mathbf{R}=\mathbf{I} \sigma_{e}^{2}$ e considerando essas estruturas de covariâncias para $\mathbf{G}$, seja $\theta$ o vetor de parâmetros em $\mathbf{V}$. Usualmente, $\theta$ é desconhecido e a aproximação de máxima verossimilhança é usada para estimá-lo (Harville, 1977; Searle et al., 1992). Esta aproximação é preferida ao tradicional método ANOVA (Searle, 1988).

As expressões (22) e (26) são as funções objetivos utilizadas pelo SAS para a estimação ML e REML, respectivamente (SAS, 1997).

Um procedimento formal para seleção de modelos para estruturas de covariâncias é utilizar os critérios: AIC (Akaike's Information Criterion), BIC (Schwatz's Bayesian Criterion) e $-2 \log L_{\mathrm{R}}(-2$ REML Log Likelihood) por meio do teste da razão de verossimilhança, (Bozdogan, 1987; Wolfinger, 1993; SAS, 1997). 
Tabela 2. Notação das estruturas de covariâncias e exemplos.

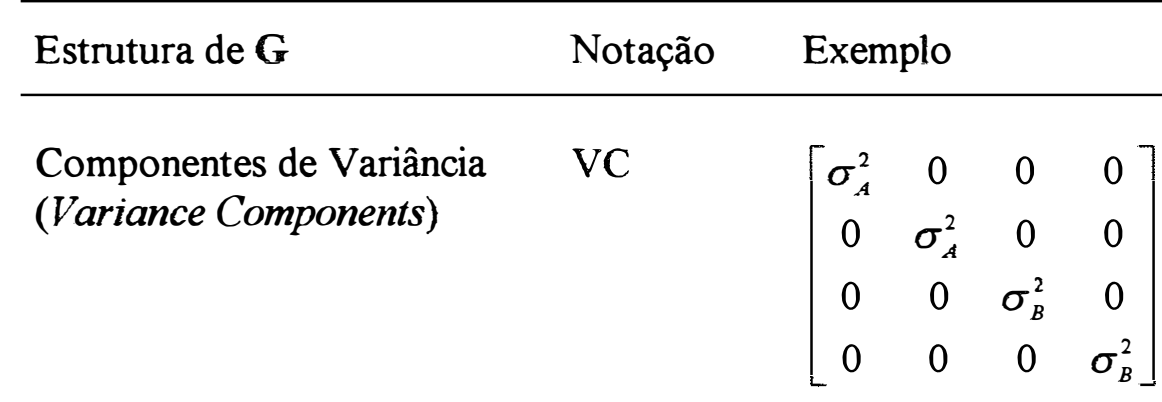

Diagonal

(Toeplitz with one band)

$\operatorname{TOEP}(1) \quad\left[\begin{array}{cccc}\sigma^{2} & 0 & 0 & 0 \\ 0 & \sigma^{2} & 0 & 0 \\ 0 & 0 & \sigma^{2} & 0 \\ 0 & 0 & 0 & \sigma^{2}\end{array}\right]$

Simetria Composta

(Compound Symmetry)

CS

$$
\left[\begin{array}{cccc}
\sigma^{2}+\sigma_{1} & \sigma_{1} & \sigma_{1} & \sigma_{1} \\
\sigma_{1} & \sigma^{2}+\sigma_{1} & \sigma_{1} & \sigma_{1} \\
\sigma_{1} & \sigma_{1} & \sigma^{2}+\sigma_{1} & \sigma_{1} \\
\sigma_{1} & \sigma_{1} & \sigma_{1} & \sigma^{2}+\sigma_{1}
\end{array}\right]
$$

HF

$$
\left[\begin{array}{ccc}
\sigma_{1}^{2} & \frac{\sigma_{1}^{2}+\sigma_{2}^{2}}{2}-\lambda & \frac{\sigma_{1}^{2}+\sigma_{3}^{2}}{2}-\lambda \\
\frac{\sigma_{1}^{2}+\sigma_{2}^{2}}{2}-\lambda & \sigma_{2}^{2} & \frac{\sigma_{2}^{2}+\sigma_{3}^{2}}{2}-\lambda \\
\frac{\sigma_{1}^{2}+\sigma_{3}^{2}}{2}-\lambda & \frac{\sigma_{2}^{2}+\sigma_{3}^{2}}{2}-\lambda & \sigma_{3}^{2}
\end{array}\right]
$$

Huynh-Feldt

(Huynh-Feldt) 


\subsection{Ajuste de modelos mistos por meio do sistema estatístico SAS}

O sistema estatístico SAS possui três procedimentos que podem ser utilizados para a análise de dados com modelos mistos: PROC GLM (General Linear Models Procedure), PROC VARCOMP (Variance Components Procedure) e PROC MIXED (Mixed Procedure) (SAS, 1990; 1992; 1996; 1997).

O PROC GLM é um procedimento utilizado para o ajuste de modelos lineares de efeitos fixos. $\mathrm{O}$ ajuste é feito através do método dos mínimos quadrados ordinários para modelos lineares. É indicado para modelos lineares balanceados e desbalanceados, assumindo sempre que todos os efeitos do modelo são fixos. Uma alternativa para a análise de modelos mistos através desse procedimento é a declaração RANDOM, que permite especificar os efeitos que são aleatórios. Tal declaração fornece os quadrados médios esperados dos efeitos do modelo, que devem ser utilizados para uma posterior correção das razões $\mathrm{F}$ do quadro da análise de variância.

O PROC VARCOMP é um procedimento utilizado para estimação dos componentes de variância em modelos lineares de efeitos aleatórios. Estima a contribuição de cada efeito aleatório para a variância da variável dependente. Como todos os efeitos nesse procedimento são considerados aleatórios, pode-se utilizá-lo para a análise de modelos mistos através da opção FIXED que permite especificar os efeitos que são fixos.

O PROC MIXED é um procedimento utilizado para ajuste do modelos lineares mistos. Permite uma especificação mais geral da matriz de variâncias e covariâncias. Ajusta o modelo misto através do método dos mínimos quadrados generalizados. É uma generalização do modelo linear padrão utilizado no PROC GLM, pois os dados podem ter correlações e variâncias não constantes. 


\subsubsection{Procedimento GLM : PROC GLM}

O procedimento GLM, do SAS, versão 6.12 (SAS, 1997), é utilizado para ajustar modelos lineares de efeitos fixos. Este procedimento utiliza o método dos mínimos quadrados para modelos lineares.

O modelo ajustado pelo PROC GLM é

$$
\mathrm{y}=\mathrm{X} \theta+e
$$

onde : y é o vetor de dados observados;

$\mathbf{X}$ é a matriz conhecida do delineamento;

$\theta$ é o vetor de parâmetros dos efeitos fixos desconhecidos;

$e$ é o vetor de erros aleatórios não observáveis, com estrutura ordinária de GaussMarkov.

Esse modelo assume que os componentes de $e$ são não correlacionados $\mathrm{e}$ normalmente distribuídos com média zero e variância $\sigma^{2}$. Assim, no modelo linear usual $e \sim \mathrm{N}\left(\phi, \mathbf{I} \sigma^{2}\right)$.

O PROC GLM é o procedimento básico do SAS para a análise de dados desbalanceados e tem a declaração RANDOM para especificar efeitos aleatórios no modelo e fornecer os quadrados médios esperados. Sempre considera todos os efeitos como fixos, mesmo quando a declaração RANDOM é utilizada para produzir os quadrados médios esperados.

Embora numerosas declarações e opções estejam disponíveis no GLM, como pode ser visto por exemplo no manual do SAS (1990), descrevem-se aqui apenas as de interesse imediato. As declarações disponíveis no GLM que serão aqui utilizadas são:

PROC GLM <opções>;

CLASS variáveis; MODEL dependentes $=$ independentes $</$ opções $>$; RANDOM efeitos </opções $>$; RUN; onde os itens entre os sinais < > são opcionais. 
A seguir, tem-se alguns comandos e opções que compõem cada uma das linhas da forma geral do PROC GLM:

\section{Declaração PROC GLM \\ PROC GLM <opções>;}

A declaração PROC GLM inicia o procedimento GLM. A opção mais utilizada na declaração PROC GLM é:

DATA = nome do arquivo: identifica o nome do arquivo de dados que será processado. Se for omitido, é considerado o arquivo criado mais recentemente.

\section{Declaração CLASS}

CLASS variáveis;

Nessa declaração são especificadas as variáveis classificatórias que serão usadas na análise. Deve aparecer antes da declaração MODEL.

\section{Declaração MODEL}

MODEL dependentes $=$ independentes $<$ lopções $>$;

Nessa declaração é definido o modelo matemático pelo qual os dados observados foram obtidos e serão analisados. Essa declaração especifica as variáveis dependentes e os efeitos independentes considerados, excluindo o termos referente a constante e ao erro. As opções mais utilizadas são:

E: fornece a forma geral de funções estimáveis.

E1, E2, E3 e E4: fornece as funções estimáveis e a soma de quadrados para os tipos I, II, II e IV, respectivamente.

SS1, SS2, SS3 e SS4: fornece as somas de quadrados dos tipos I, II, III e IV, respectivamente.

No SAS, se nenhum tipo de soma de quadrados for especificado, o GLM fornece as somas dos tipos I e III, por default. 
Quando especificado, o PROC GLM fornece as somas de quadrados dos tipos I, II, III, e IV, que estão exemplificadas na Tabela 1, para um modelo com dois fatores fixos e interação (Iemma, 1997a).

Tabela 1. Somas de quadrados segundo o PROC GLM, de acordo com a causa de variação e o tipo.

\begin{tabular}{lllll}
\hline $\begin{array}{l}\text { Causa de } \\
\text { Variação }\end{array}$ & Tipo I & Tipo II & Tipo III & Tipo IV \\
\hline A (linhas) & $\mathrm{R}(\alpha \mid \mu)$ & $\mathrm{R}(\alpha \mid \mu, \beta)$ & $\mathrm{R}(\alpha \mid \mu, \beta, \gamma)$ & Contrastes \\
$\mathrm{B}$ (colunas) & $\mathrm{R}(\beta \mid \mu, \alpha)$ & $\mathrm{R}(\beta \mid \mu, \alpha)$ & $\mathrm{R}(\beta \mid \mu, \alpha, \gamma)$ & Contastes \\
$\mathrm{A} * \mathrm{~B}$ (interação) & $\mathrm{R}(\gamma \mid \mu, \alpha, \beta)$ & $\mathrm{R}(\gamma \mid \mu, \alpha, \beta)$ & $\mathrm{R}(\gamma \mid \mu, \alpha, \beta)$ & $\mathrm{R}(\gamma \mid \mu, \alpha, \beta)$ \\
\hline
\end{tabular}

\section{As hipóteses mais comuns sobre efeitos de linhas são:}

TIPO I: Hipóteses sobre médias ponderadas de linhas, $\overline{\mathrm{L}}_{\mathrm{i}}$.

$$
\mathrm{H}_{0}^{(1-\mathrm{A})}: \quad \overline{\mathrm{L}}_{\mathrm{i}}=\overline{\mathrm{L}}_{\overrightarrow{\mathrm{i}}} \quad \forall\left(\mathrm{i}, \mathrm{i}^{\prime}\right)
$$

com

$$
\mathrm{SQH}_{0}^{(1-\mathrm{A})}=\mathrm{R}(\alpha \mid \mu)
$$

TIPO II: Hipóteses sobre médias ponderadas de linhas ajustadas para colunas, $\overline{\mathbf{f}}_{\mathrm{i}}$.

$$
\mathrm{H}_{0}^{(2-\mathrm{A})}:{\overline{\xi_{i}}}_{\mathrm{i}}={\overline{\boldsymbol{E}_{\mathrm{i}}}}_{\mathrm{i}} \quad \forall\left(\mathrm{i}, \mathrm{i}^{\prime}\right)
$$

com

$$
\mathrm{SQH}_{0}^{(2-\mathrm{A})}=\mathrm{R}(\alpha \mid \mu, \beta)
$$

TIPO III: Hipóteses sobre médias não ponderadas de linhas, $\bar{l}_{\mathrm{i}}$.

$$
\mathrm{H}_{0}^{(3-\mathrm{A})}: \quad \overline{\mathrm{l}}_{\mathrm{i}}=\overline{\mathrm{l}}_{\mathrm{i}^{\prime}} \quad \forall\left(\mathrm{i}, \mathrm{i}^{\prime}\right)
$$

com

$$
\mathrm{SQH}_{0}{ }^{(3-\mathrm{A})}=\mathrm{R}(\alpha \mid \mu, \beta, \gamma)
$$


TIPO IV: Hipóteses sobre médias não ponderadas de linhas, por meio de contrastes entre médias de mesma coluna, iniciando-se sempre pela última linha. As hipóteses do tipo IV são similares as do tipo III, se não existem caselas vazias. Se, no entanto, existe ao menos uma casela vazia e o modelo contém interação, então as hipóteses dos tipos III e IV são em geral diferentes. As hipóteses do tipo IV, não são habitualmente descritas através da notação $\mathrm{R}(\cdot)$.

Ademais, se existem caselas vazias, as hipótese do tipo IV podem não ser únicas pois, elas dependem da posição e do número de caselas vazias (Iemma, 1995a; 1995b; Iemma \& Perri, 1997).

Analogamente tem-se as hipóteses mais comuns sobre efeitos de colunas:

TIPO I : Hipóteses sobre médias ponderadas de colunas, $\overline{\mathbf{C}}_{\mathbf{j}}$.

$$
\mathrm{H}_{0}^{(1-B)}: \quad \bar{C}_{\mathrm{j}}=\overline{\mathrm{C}}_{\mathrm{j}^{\prime}} \quad \forall\left(\mathrm{j}, \mathrm{j}^{\prime}\right)
$$

com

$$
\mathrm{SQH}_{0}{ }^{(1-B)}=\mathbf{R}(\beta \mid \mu)
$$

TIPO II: Hipóteses sobre médias ponderadas de colunas ajustadas para linhas, $\bar{\epsilon}_{\mathrm{j}}$.

$$
\mathrm{H}_{0}^{(2-\mathrm{B})}: \bar{\epsilon}_{\mathrm{j}}=\bar{\epsilon}_{\mathrm{j}^{\prime}} \quad \forall\left(\mathrm{j}, \mathrm{j}^{\prime}\right)
$$

com

$$
\mathrm{SQH}_{0}^{(2-B)}=\mathrm{R}(\beta \mid \mu, \alpha)
$$

TIPO III: Hipóteses sobre médias não ponderadas de colunas, $\overline{\mathrm{c}}_{\mathrm{j}}$.

$$
\mathrm{H}_{0}^{(3-B)}: \quad \overline{\mathrm{c}}_{\mathrm{j}}=\overline{\mathrm{c}}_{\mathrm{j}^{\prime}} \quad \forall\left(\mathrm{j}, \mathrm{j}^{\prime}\right)
$$

com

$$
\mathrm{SQH}_{0}^{(3-B)}=\mathrm{R}(\gamma \mid \mu, \alpha, \beta)
$$

TIPO IV: Hipóteses sobre médias não ponderadas de colunas, por meio de contrastes entre médias de mesma linha, iniciando-se sempre pela última coluna. Conforme dito anteriormente as hipóteses do tipo IV são similares as do tipo III, se não existem caselas vazias. 


\section{Hipóteses sobre a interação:}

$$
\mathrm{H}_{0}^{(\mathrm{AB})}: \mu_{\mathrm{ij}}-\mu_{\mathrm{i}^{\prime} \mathrm{j}}-\mu_{\mathrm{ij}}+\mu_{\mathrm{i}^{\prime} \mathrm{j}^{\prime}}=0
$$

com

$$
\mathrm{SQH}_{0}^{(\mathrm{AB})}=\mathrm{R}(\gamma \mid \mu, \alpha, \beta)
$$

Se todas as caselas são ocupadas, o "teste da interação" pode ser utilizado como critério de escolha entre um modelo com e sem interação, pois nesse caso estão presentes todas as interações:

$$
\mathrm{H}_{0}{ }^{(\mathrm{AB})}: \Delta_{\mathrm{i} i}{ }^{\prime} \mathrm{j}^{\prime}=0 \quad \text { para qualquer } \mathrm{i}, \mathrm{i}^{\prime}, \mathrm{j}, \mathrm{j} \text { ' }
$$

Se há caselas vazias, o "teste da interação" não deve ser utilizado como critério para tal escolha (Iemma, 1991).

\section{Declaração RANDOM}

\section{RANDOM efeitos </opções $>$;}

Essa declaração especifica quais, dentre os efeitos do modelo, são aleatórios. Quando a declaração RANDOM é utilizada, o GLM fornece os quadrados médios esperados para todos os efeitos do modelo. Por default o PROC GLM fornece os quadrados médios esperados do tipo III (SAS, 1990).

A opção mais utilizada é:

TEST: efetua testes de hipóteses para cada efeito especificado no modelo, utilizando os termos do erro apropriado, conforme determinado pelos quadrados médios esperados. Essa opção é utilizada para compor o denominador do teste "F" para cada causa de variação.

O PROC GLM não declara automaticamente interações como aleatórias mesmo quando os efeitos da interação são declarados aleatórios. Por exemplo, random a b/ test;

não produz os mesmos quadrados médios esperados ou testes que random a b a*b/test; 
Para assegurar testes corretos, todas as interações aleatórias e todos os efeitos principais aleatórios devem ser listados na declaração RANDOM.

\section{Declaração RUN;}

Executa o processamento da análise de dados.

\subsubsection{Procedimento VARCOMP : PROC VARCOMP}

O PROC VARCOMP é um procedimento para estimação dos componentes de variância em um modelo linear geral. Esse procedimento é apropriado para modelos que têm efeitos aleatórios e estima a contribuição de cada efeito aleatório para a variância da variável dependente (SAS, 1990).

Dentre as declarações disponíveis no PROC VARCOMP, as seguintes são aqui utilizadas:

PROC VARCOMP <opções >;

CLASS variáveis;

Model dependentes $=$ efeitos $</ o p c ̧ a ̃ o>$; RUN;

Somente uma declaração MODEL é permitida. As declarações MODEL e CLASS são descritas depois da declaração PROC VARCOMP.

\section{Declaração PROC VARCOMP}

PROC VARCOMP < opções>;

As seguintes opções podem aparecer:

DATA $=$ nome do arquivo

Identifica o nome do arquivo de dados que será processado. Se for omitido, é considerado o arquivo criado mais recentemente. 


\section{METHOD $=$ TYPE1 $\mid$ MIVQUE0 $\mid$ ML $\mid$ REML}

Especifica qual dos quatro métodos disponiveis no PROC VARCOMP: TYPE1, MIVQUE0, ML e REML será usado.

Os quatro métodos de estimação que podem ser especificados nesse procedimento são descritos a seguir:

TYPE1: Este método baseia-se nas somas de quadrados do tipo I ou ANOVA, obtidas pelo método III de Henderson. Cada quadrado médio que envolve apenas efeitos aleatórios é igualado ao seu valor esperado.

MIVQUE0: Similar ao anterior, exceto pelo fato de que os efeitos aleatórios são ajustados somente pelos efeitos fixos. É o método default do PROC VARCOMP.

ML: Método da máxima verossimilhança. Utiliza os valores iniciais obtidos através do MIVQUE0 e o procedimento é iterativo até que a função logverossimilhança convirja.

REML: Método de máxima verossimilhança restrita. Separa conforme descrito em 4.2.2.6, a função de verossimilhança em duas partes: uma contém os efeitos fixos e a outra é livre dos efeitos fixos. É também um método iterativo, que inicia o procedimento com as estimativas MIVQUE0 até a função log-verossimilhança, da parte que não contém os efeitos fixos, convergir.

Os métodos ML e REML não fornecem componentes de variância negativos e fornecem a matriz de covariâncias assintóticas para os componentes.

\section{Declaração CLASS}

CLASS variáveis;

Nessa declaração são especificadas as variáveis classificatórias que serão utilizadas na análise. Todos os efeitos na declaração MODEL devem ser compostos dos efeitos que aparecem na declaração CLASS. 


\section{Declaração MODEL}

Model dependentes $=$ efeitos $</$ opção $>$;

A declaração MODEL especifica as variáveis dependentes e os efeitos independentes. Todos os efeitos independentes devem se compostos de efeitos que aparecem na declaração CLASS. Somente uma declaração MODEL é permitida.

Somente uma opção é disponível na declaração MODEL:

$$
\text { FIXED }=\mathrm{n}
$$

Especifica que os $\mathrm{n}$ primeiros efeitos na declaração MODEL são efeitos fixos. Os efeitos restantes são considerados aleatórios. Por default, PROC VARCOMP assume que todos os efeitos são aleatórios. Se a notação barra for usada, por exemplo, $\mathrm{y}=\mathrm{A} \mid \mathrm{B} / \mathrm{F}[\mathrm{XED}=2$, então $\mathrm{A} * \mathrm{~B}$ é considerado um efeito aleatório.

\subsubsection{Procediment MIXED : PROC MIXED}

$\mathrm{O}$ procedimento MIXED ajusta o modelo linear misto, do tipo $\mathrm{y}=\mathbf{X} \theta+\mathbf{Z u}+\boldsymbol{e}$ como uma generalização do modelo linear utilizado no procedimento GLM.

O modelo linear misto, portanto, tem a flexibilidade de modelar não somente as médias dos dados, como no modelo linear de efeitos fixos, mas também suas variâncias e covariâncias. Permite uma especificação mais geral da matriz de covariâncias de u e de $e$. $\mathrm{O}$ modelo linear misto também permite que os componentes de u e de $e$ sejam correlacionados.

Para esse modelo tem-se, como em (4)

$$
\begin{aligned}
& \mathrm{E}(\mathrm{u})=\left[\begin{array}{l}
\mathrm{u} \\
e
\end{array}\right]=\mathbf{0} \\
& \operatorname{var}\left[\begin{array}{l}
\mathrm{u} \\
e
\end{array}\right]=\left[\begin{array}{ll}
\mathbf{G} & 0 \\
0 & \mathbf{R}
\end{array}\right]
\end{aligned}
$$


Ou seja

$$
\mathrm{y} \sim \mathrm{N}(\mathbf{X} \boldsymbol{\theta}, \mathbf{Z G Z}+\mathbf{R})
$$

A variância de $\mathbf{y}$ é então $\mathbf{V}=\mathbf{Z G Z}+\mathbf{R}$. Pode-se construir $\mathbf{V}$ montando-se a matriz de efeitos aleatórios $\mathbf{Z}$ e especificando-se estruturas de variâncias e covariâncias para $\mathbf{G}$ e $\mathbf{R}$.

Embora esta seja uma especificação geral do modelo linear misto, aqui será considerado o caso no qual $\mathbf{R}=\mathbf{I} \boldsymbol{\sigma}^{2}$.

O modelo linear padrão para efeitos fixos apresentado em (1) pode ser visto como um caso particular do modelo linear misto, com:

$$
\mathbf{Z}=\phi \quad \text { e } \quad \mathbf{R}=\mathbf{I} \sigma^{2}
$$

Dentre as declarações disponíveis no PROC MIXED (SAS, 1997), as seguintes são aqui utilizadas.

PROC MIXED <opções >;

CLASS varióveis;

MODEL dependente $=<$ efeitos fixos $></$ opções $>$;

RUN;

RANDOM efeitos aleatórios $</$ opções $>$;

As declarações PROC MIXED e MODEL são exigidas, e a declaração MODEL deve aparecer depois da declaração CLASS.

\section{Declaração PROC MIXED}

PROC MIXED <opções >;

As seguintes opções podem aparecer:

DATA $=$ nome do arquivo

Identifica, como nos procedimentos anteriores, o nome do arquivo de dados que será processado. Se for omitido é considerado o arquivo criado mais recente.

\section{METHOD $=$ REML $\mid$ ML $\mid$ MIVQUEO}

Especifica o método de estimação para os componentes de variância. $\mathrm{O}$ método REML é o método default. 


\section{Declaração CLASS}

CLASS variáveis;

Nessa declaração são especificadas as variáveis classificatórias que serão utilizadas na análise.

\section{Declaração MODEL}

MODEL dependentes $=<$ efeitos-fixos $></$ opções $>$;

Nessa declaração é definida a variável dependente e os efeitos fixos, que determinam a matriz $\mathbf{X}$, da parte fixa do modelo misto.

As seguintes opções podem ser especificadas na declaração MODEL:

$\mathbf{E}$ : fornece a forma geral das funções estimáveis.

E1, E3: fornece as funções estimáveis para os tipos I e III, respectivamente

HTYPE = 1, 3: fornece os testes de hipóteses dos tipos I e III para todos os efeitos fixos, respectivamente. Tipo III é o default.

\section{Declaração RANDOM}

RANDOM efeitos aleatórios $</$ opções $>$;

Essa declaração define a matriz $\mathbf{Z}$, da parte aleatória do modelo misto, os efeitos aleatórios que constituem o vetor $\mathbf{u}$, e a estrutura de $\mathbf{G}$.

Uma das opções mais importantes do comando RANDOM é TYPE = estrutura, que especifica a estrutura de $\mathbf{G}$. A versão 6.12 do SAS fornece várias opções sobre tais estruturas (SAS, 1997). Quando TYPE = estrutura, não é especificado, a estrutura VC (Variance Components) é assumida por default.

Outra opção disponível é GDATA = SAS-data-set, especifica que a matriz G, conhecida, seja lida de um SAS data set.

Neste trabalho serão consideradas as estruturas já apresentadas em 4.2.5 . 


\subsubsection{Procedimento MIXED versus procedimento GLM}

- O PROC MIXED é uma generalização do PROC GLM no sentido de que o PROC GLM ajusta o modelo linear padrão e o PROC MIXED ajusta modelos lineares mistos.

- Eles têm sintaxe similar, ambos têm as mesmas declarações básicas: CLASS, MODEL, CONTRAST, ESTIMATE e LSMEANS.

- Eles usam a mesma parametrização (parametrização do modelo superparametrizado de posto incompleto)

- O PROC MIXED fornece somente testes dos tipos I e III para os efeitos fixos, enquanto o PROC GLM fornece testes dos tipos I, II, III, e IV.

- A declaração RANDOM no PROC MIXED incorpora os efeitos aleatórios constituindo o vetor u no modelo misto, enquanto que no PROC GLM, os efeitos especificados na declaração RANDOM são ainda tratados como fixos no ajuste do modelo e servem para produzir os quadrados médios esperados correspondentes, que conduzem às tradicionais estimativas ANOVA dos componentes de variância.

- O PROC GLM fornece as estimativas dos componentes de variância por meio do método ANOVA, e o PROC MIXED fornece as estimativas REML e ML dos componentes de variância, as quais são geralmente preferidas às estimativas ANOVA. Opcionalmente, o PROC MIXED também fornece as estimativas MIVQUEO dos componentes de variância, as quais são similares às estimativas ANOVA. 


\subsubsection{Procedimento MIXED versus procedimento VARCOMP}

- O PROC MIXED generaliza o PROC VARCOMP, mas não inclui as estimativas tipo I, estimativas ANOVA baseadas no método III de Henderson, dos componentes de variância.

- Ambos fornecem as estimativas REML, ML e MIVQUEO dos componentes de variância.

- O PROC VARCOMP não fornece as estimativas dos efeitos fixos.

- O PROC MIXED tem como opções uma ampla variedade de estruturas de covariâncias como visto em 4.2.5, enquanto que o PROC VARCOMP utiliza apenas a matriz VC.

- O PROC MIXED gera diversas análises que estão ausentes no PROC VARCOMP, incluindo a estimação e testes de combinações lineares de efeitos fixos e aleatórios. 


\section{DISCUSSÃO DE UM EXEMPLO}

Apresenta-se a seguir um exemplo procurando discutir e comparar os resultados fornecidos pelos procedimentos GLM; VARCOMP e MIXED do SAS, evidenciando as hipóteses estatísticas sobre os efeitos fixos que estão sendo testadas na análise de variância para o modelos mistos desbalanceados bem como as estimativas dos componentes de variância (Iemma, 1997b; Iemma \& Perri, 1996).

\subsection{Descrição dos dados}

O exemplo a seguir é constituído de um conjunto de dados adaptados do Programa de Desenvolvimento Ponderal de Bovinos da Raça Canchim, gentilmente cedidos pelo Prof. Dr. Alfredo Ribeiro de Freitas, com o objetivo de ilustrar a utilização dos procedimentos GLM, VARCOMP e MIXED, no ajuste de modelos mistos desbalanceados com dois fatores (A fixo e B aleatório) e com interação.

Os dados referem-se aos pesos aos 12 meses de idade, ajustados linearmente para 365 dias, de 22 bezerros da raça Canchim, classificados de acordo com o sexo do bezerro e o número do touro (Freitas, 1991).

O modelo adotado para descrever os dados é o modelo misto desbalanceado com dois fatores (Sexo fixo e Touro aleatório) e com interação. 
Os dados estão apresentados na Tabela 3.

Tabela 3. Pesos aos 12 meses de idade, em $\mathrm{kg}$, de bezerros da raça Canchim

\begin{tabular}{cccc}
\hline \multirow{2}{*}{$\begin{array}{c}\text { Fator A } \\
\text { Sexo }\end{array}$} & $\mathrm{j}=1$ & $\mathrm{j}=2$ & $\mathrm{j}=3$ \\
\cline { 2 - 4 } $\mathrm{i}=1$ & 176 & 153 & 207 \\
Fêmea & 229 & 173 & 177 \\
& 197 & 189 & 185 \\
& 212 & 195 & 220 \\
$\mathrm{i}=2$ & 190 & - & - \\
Macho & 235 & 220 & 230 \\
& 266 & 252 & 249 \\
& 280 & - & 199 \\
& 293 & - & - \\
\hline
\end{tabular}

Fonte: Dados adaptados do Centro de Pesquisa de Pecuána do Sudeste - CPPSE/EMBRAPA, São Carlos, SP.

O modelo misto desbalanceado com dois fatores, pode ser caracterizado pelo modelo superparametrizado:

$$
\mathrm{y}_{\mathrm{ijk}}=\mu+\alpha_{\mathrm{i}}+\beta_{\mathrm{j}}+\gamma_{\mathrm{ij}}+\varepsilon_{\mathrm{ijk}}
$$

com $i=1,2 ; j=1,2,3$ e $k=1,2, \ldots, \mathrm{n}_{\mathrm{ij}}$

onde: $y_{i j k}$ é o peso do animal;

$\mu$ é uma constante inerente à todas as observações;

$\alpha_{i} \quad$ é o efeito fixo do sexo $\mathrm{i}$;

$\beta_{\mathrm{j}}$ é o efeito aleatório do touro j onde $\beta_{\mathrm{j}} \sim \mathrm{N}\left(0, \sigma_{\beta}{ }^{2}\right)$ e independentes;

$\gamma_{\mathrm{ij}}$ é o efeito da interação touros e sexos onde $\gamma_{\mathrm{ij}} \sim \mathrm{N}\left(0, \sigma_{\gamma}{ }^{2}\right)$ e independentes;

$\varepsilon_{\mathrm{ijk}}$ é o erro aleatório onde $\varepsilon_{\mathrm{ijk}} \sim \mathrm{N}\left(0, \sigma_{e}{ }^{2}\right)$ e independentes.

$\beta_{\mathrm{j}}, \gamma_{\mathrm{ij}}$ e $\varepsilon_{\mathrm{ijk}}$ são independentes.

Assim:

$$
\mathrm{y} \sim \mathrm{N}\left(\mathbf{X} \beta, \mathbf{Z G Z} \mathbf{G}^{\prime}+\mathbf{I}_{e}{ }^{2}\right) \quad \text { onde } \quad \mathbf{G}=\left[\begin{array}{cc}
\mathbf{I}_{3} \sigma_{\beta}^{2} & \phi \\
\phi & \mathbf{I}_{6} \sigma_{\gamma}^{2}
\end{array}\right]
$$


Para o conjunto de dados do Tabela 3, tem-se:

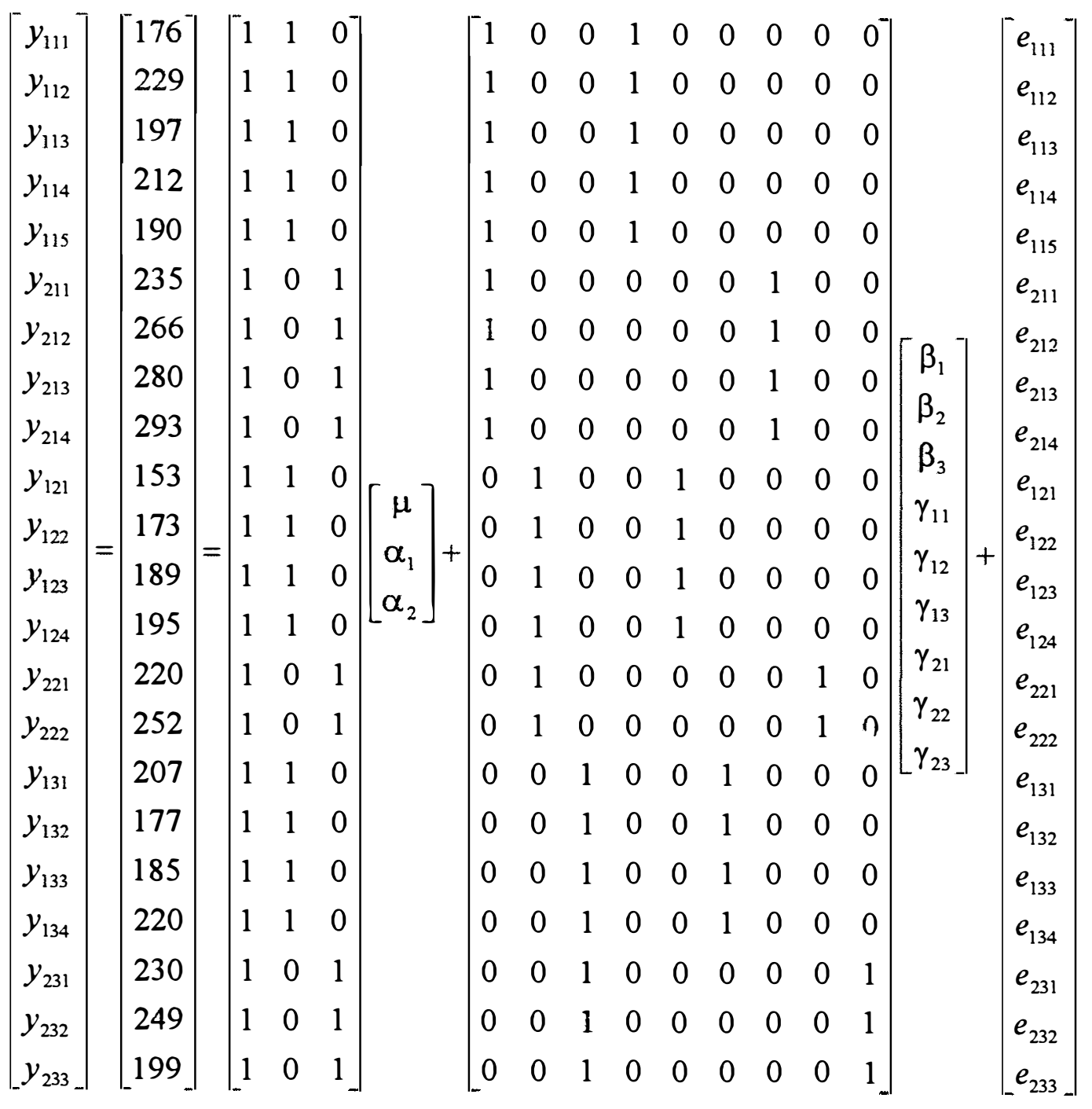

A seguir tem-se os programas SAS e suas respectivas saídas para ajustar os dados da Tabela 3. 


\subsubsection{Procedimento GLM}

Segundo Iemma (1995a), Perri \& Iemma (1996), conforme o interesse do pesquisador, o modelo superparametrizado pode assumir diferentes caracterizações, desde o modelo mais simples até o modelo completo.

(i) $y_{i}=\mu+e_{i}$

(ii) $y_{i j}=\mu+\alpha_{i}+e_{i j}$

(iii) $y_{i j}=\mu+\beta_{j}+e_{i j}$

(iv) $y_{i j k}=\mu+\alpha_{i}+\beta_{j}+e_{i j k}$

(v) $y_{i j k}=\mu+\alpha_{i}+\beta_{j}+\gamma_{i j}+\varepsilon_{i j k}$ $\rightarrow \quad y_{i}=\mu+e_{i}$

$\rightarrow \quad y_{i j}=\mu_{i}+e_{i j}$

$\rightarrow \quad \mathrm{y}_{\mathrm{ij}}=\mu_{\mathrm{j}}+\mathrm{e}_{\mathrm{ij}}$

$\rightarrow \quad y_{i j k}=\mu_{i}+\beta_{j}+e_{i j k}$

$\rightarrow \quad y_{i j k}=\mu_{i j}+\varepsilon_{i j k}$

Esses são, respectivamente, o modelo-S e modelo-M (modelo superparametrizado e modelo de médias de caselas, respectivamente).

Considerando os dados da Tabela 3 tem-se esquematicamente, para o modelo-S e para o modelo-M, as Tabelas 4 e 5 respectivamente.

Tabela 4. Esquema para o modelo-S.

Fator A

Fator $\mathrm{B}=$ Touro

Sexo

$\mathrm{j}=1$
$\mathrm{j}=2$ $\mathrm{j}=3$

$\mathrm{i}=1 \quad \mu+\alpha_{1}+\beta_{1}+\gamma_{11}(5)$

$\mu+\alpha_{1}+\beta_{2}+\gamma_{12}(4)$

$\mu+\alpha_{1}+\beta_{3}+\gamma_{13}$

$\mathrm{i}=2 \quad \mu+\alpha_{2}+\beta_{1}+\gamma_{21}(4)$

$\mu+\alpha_{2}+\beta_{2}+\gamma_{22}(2)$

$\mu+\alpha_{2}+\beta_{3}+\gamma_{23}(3)$

Tabela 5. Esquema para o modelo-M.

\begin{tabular}{cccc} 
Fator A & \multicolumn{3}{c}{ Fator B = Touro } \\
\cline { 2 - 4 } Sexo & $\mathrm{j}=1$ & $\mathrm{j}=2$ & $\mathrm{j}=3$ \\
$\mathrm{i}=1$ & $\mu_{11}(5)$ & $\mu_{12}(4)$ & $\mu_{13}(4)$ \\
$\mathrm{i}=2$ & $\mu_{21}(4)$ & $\mu_{22}(2)$ & $\mu_{23}(3)$ \\
\hline
\end{tabular}




\subsubsection{Um programa}

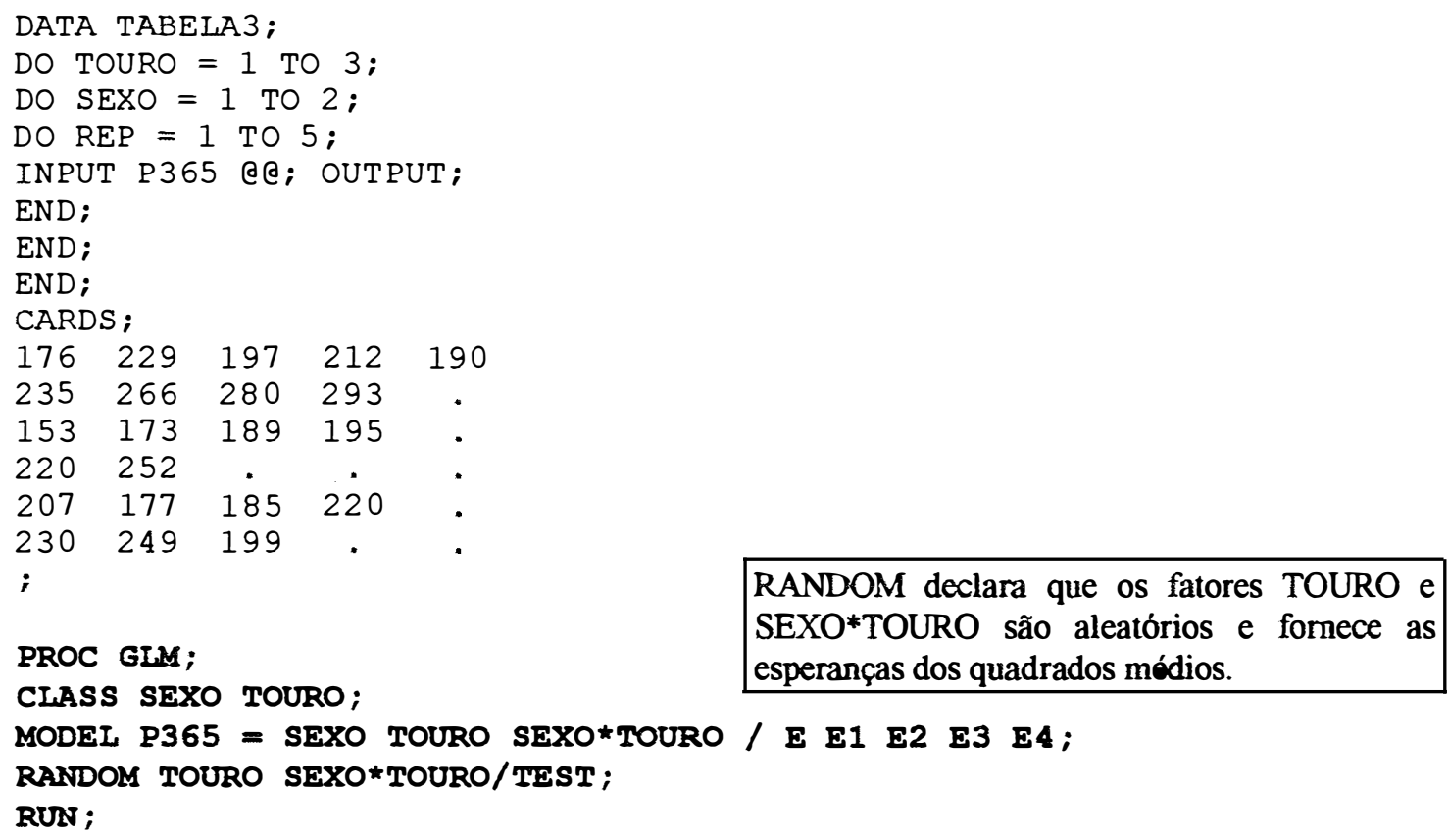

\subsubsection{Funções estimáveis e hipóteses testáveis}

O GLM fornece as funções estimáveis solicitadas no MODEL, para cada um dos efeitos, considerando todos os efeitos como fixos, apesar da opção RANDOM.

O GLM constrói uma forma geral para as funções estimáveis por meio de $\left(\mathbf{X}^{\prime} \mathbf{X}\right)^{\mathbf{g}^{2}}\left(\mathbf{X}^{\mathbf{X}} \mathbf{X}\right)$ usando suas linhas não nulas para representar $\mathrm{L}$, onde $\left(\mathbf{X}^{\mathbf{\prime}} \mathbf{X}\right)^{\mathrm{g}^{2}}$ é inversa generalizada reflexiva de (X'X). (Iemma, 1997a; Mondardo \& Iemma, 1998).

A saída da opção $\mathrm{E}$, forma geral das funções estimáveis, da declaração MODEL do PROC GLM é mostrada na Tabela 6. 
Tabela 6. Forma geral das funções estimáveis.

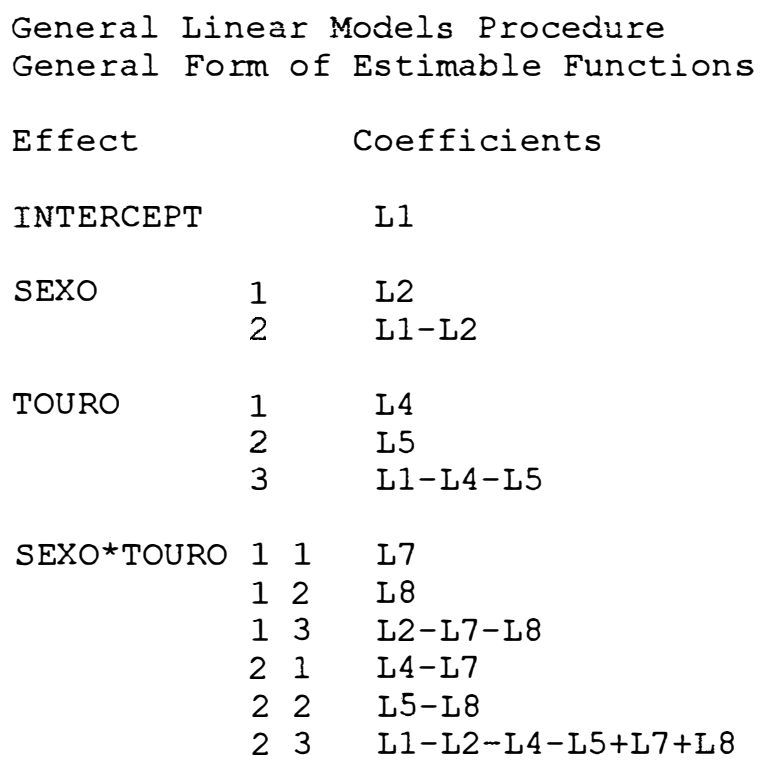

Atribuindo-se valores aos coeficientes $L$ 's, obtém-se as formas específicas das funções estimáveis.

Conforme visto em 4.3.1, a hipótese tipo I para sexo (fator A) é uma hipótese sobre médias ponderadas de linhas. Para os dados da Tabela 3, pode ser escrita utilizando-se a Tabela 5 e o modelo-M, como:

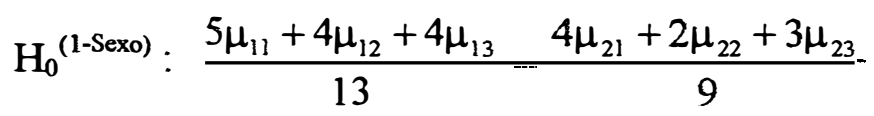

Como o PROC GLM do SAS utiliza o modelo superparametrizado, modelo-S, substituindo-se $\mu_{\mathrm{ij}}$ por $\mu+\alpha_{\mathrm{i}}+\beta_{\mathrm{j}}+\gamma_{\mathrm{ij}}, \mathrm{H}_{0}^{(1-\text { Sexo) }}$ resulta em:

$$
\begin{aligned}
\mathrm{H}_{0}^{(1-S e x o)}: & \alpha_{1}-\alpha_{2}-\frac{7}{117} \beta_{1}+\frac{10}{\omega 7} \beta_{2}-\frac{3}{17} \beta_{3}+\frac{45}{17} \gamma_{11}+\frac{36}{117} \gamma_{12}+ \\
+ & \frac{36}{17} \gamma_{13}-\frac{52}{117} \gamma_{21}-\frac{26}{117} \gamma_{22}-\frac{39}{117} \gamma_{23}=0
\end{aligned}
$$

Como pode ser visto na Tabela 7, fazendo-se $L 2=1$, nos coeficientes das funções estimáveis do tipo I, para o fator sexo, obtém-se a forma equivalente: 


$$
\begin{aligned}
\mathrm{H}_{0}{ }^{(1-\operatorname{Sexo})}: & \alpha_{1}-\alpha_{2}-0,0598 \beta_{1}+0,0855 \beta_{2}-0,0256 \beta_{3}+0,3846 \gamma_{11}+0,3077 \gamma_{12}+ \\
& +0,3077 \gamma_{13}-0,4444 \gamma_{21}-0,2222 \gamma_{22}-0,3333 \gamma_{23}=0
\end{aligned}
$$

Com raciocínio análogo, pode-se converter todas as demais hipóteses deste estudo, do modelo-M para o modelo-S. Naturalmente, a apresentação das hipóteses no modelo-M pode simplificar sobremaneira a sua interpretação.

Observa-se nas Tabelas 7 e 8, que as funções estimáveis do tipo I para touro, são na realidade do tipo $I$, de acordo com 4.3.1, pois são ajustadas para sexo.

Para a obtenção da hipótese do tipo I para touro, o fator B deveria ser posto no modelo, antes do fator $A$, para que fosse possível obter $R(\beta \mid \mu)$.

Verifica-se também, que a hipótese tipo I para sexo tem um único grau de liberdade, envolvendo um único $L: L 2$. De modo análogo a hipótese tipo I para touro tem dois graus de liberdade, pois é constituída de dois $L$ 's: $L 4$ e $L 5$, e a hipótese para a interação tem também dois graus de liberdade, é também constituída de dois $L$ 's: $L 7$ e $L 8$.

As funções estimáveis dos tipos I, II, III e IV para cada um dos efeitos considerados: sexo, touro e sexo*touro, e as respectivas hipóteses que estão sendo testadas, conforme já mencionado no item 4.3.1, estão descritas nas Tabelas 7 a 10. 
Tabela 7. Funções estimáveis do tipo I e suas respectivas hipóteses para os modelos $\mathrm{S}$ e $\mathrm{M}$.

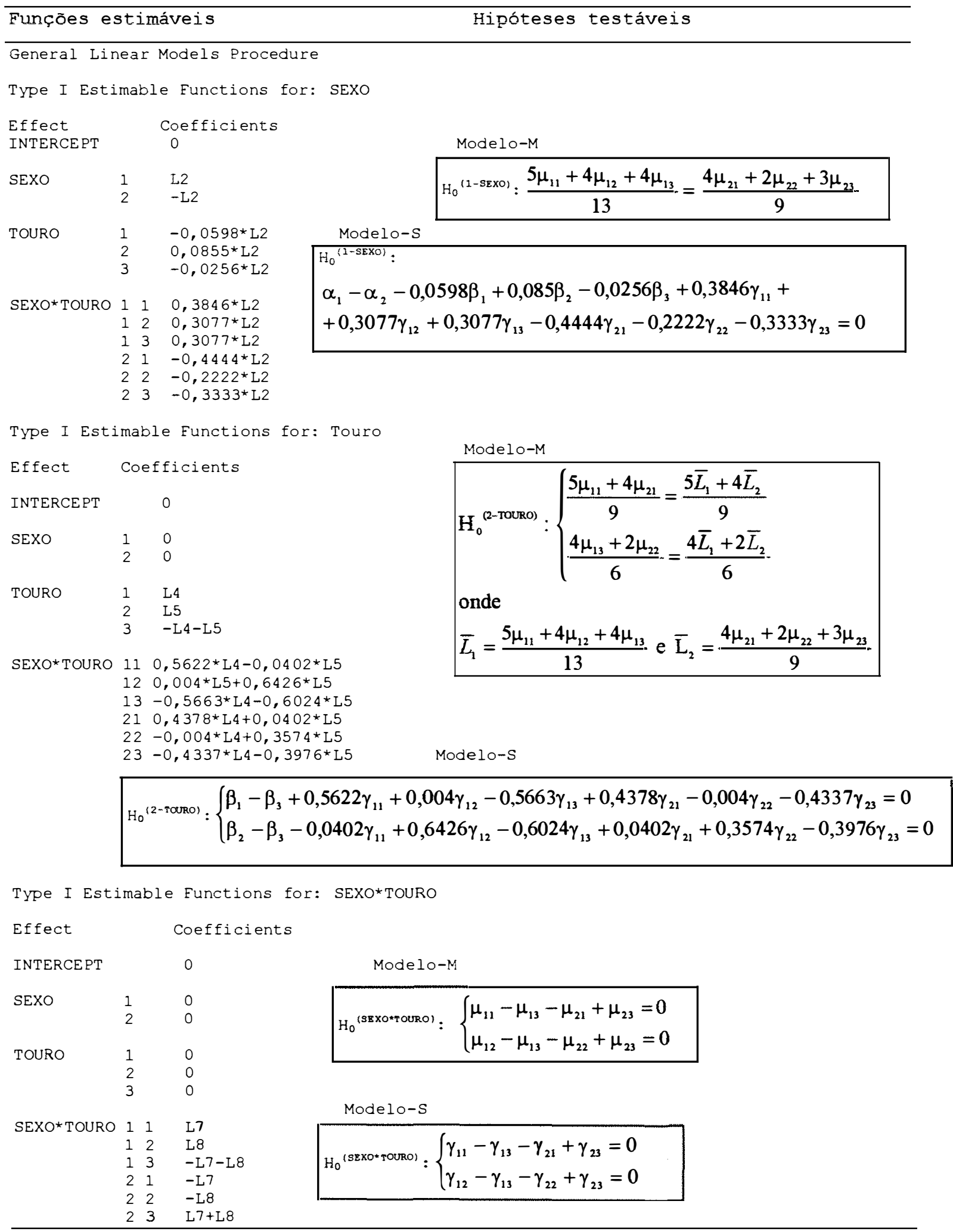


Tabela 8. Funções estimáveis do tipo II e suas respectivas hipóteses para os modelos $\mathrm{S}$ e $\mathrm{M}$.

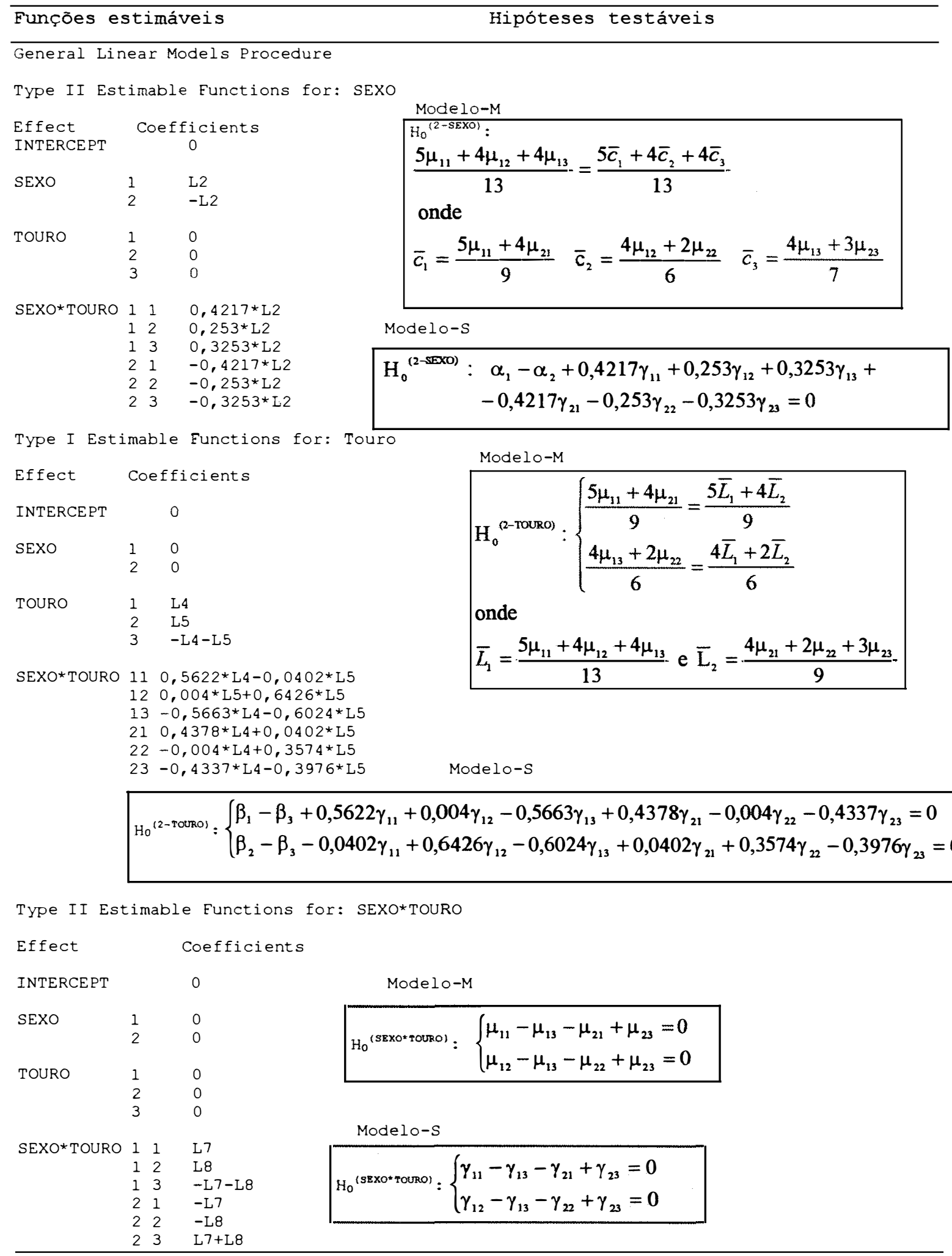


Tabela 9. Funções estimáveis do tipo III e suas respectivas hipóteses para os modelos $\mathrm{S}$ e M.

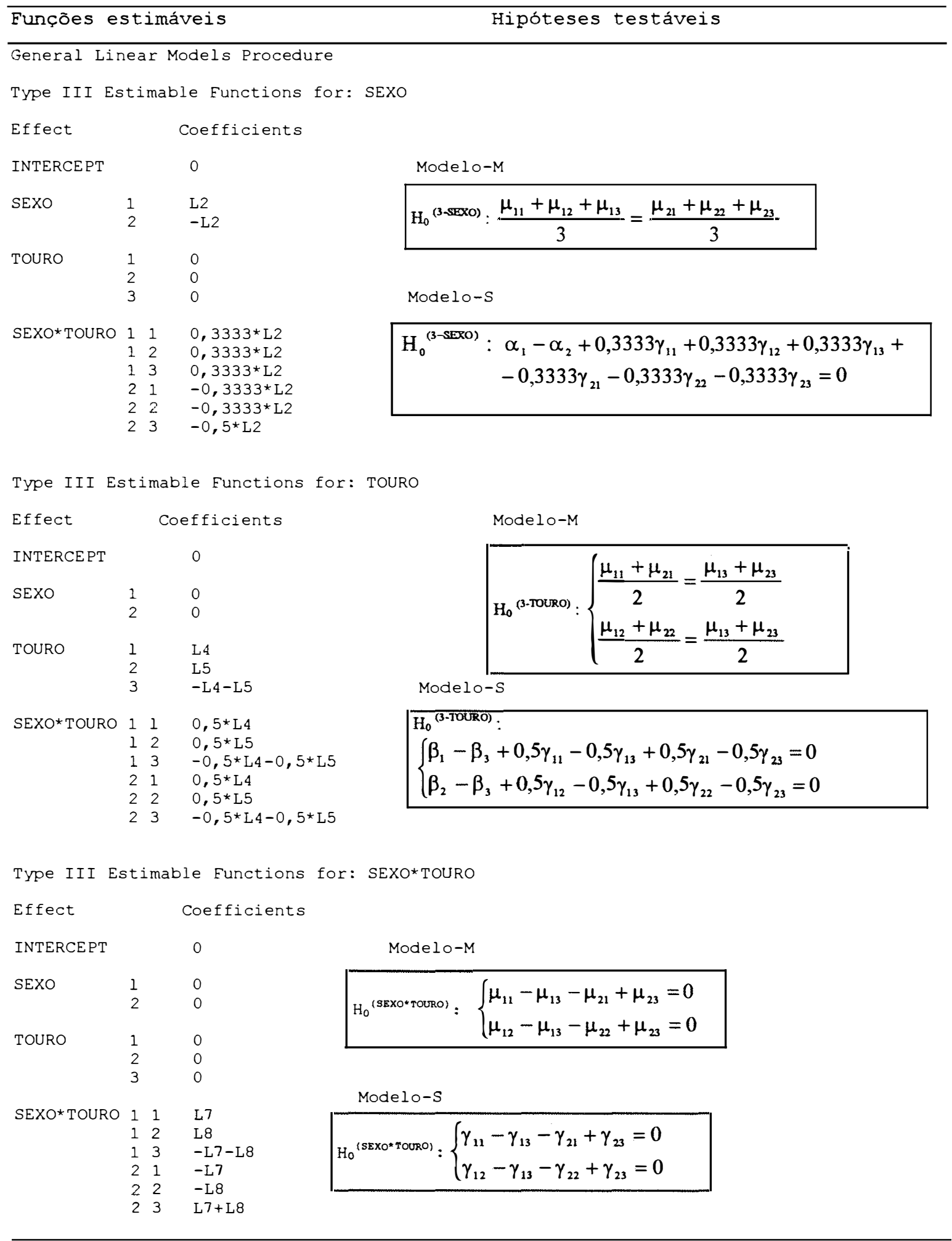


Tabela 10. Funções estimáveis do tipo IV e suas respectivas hipóteses para os modelos $\mathrm{S}$ e M.

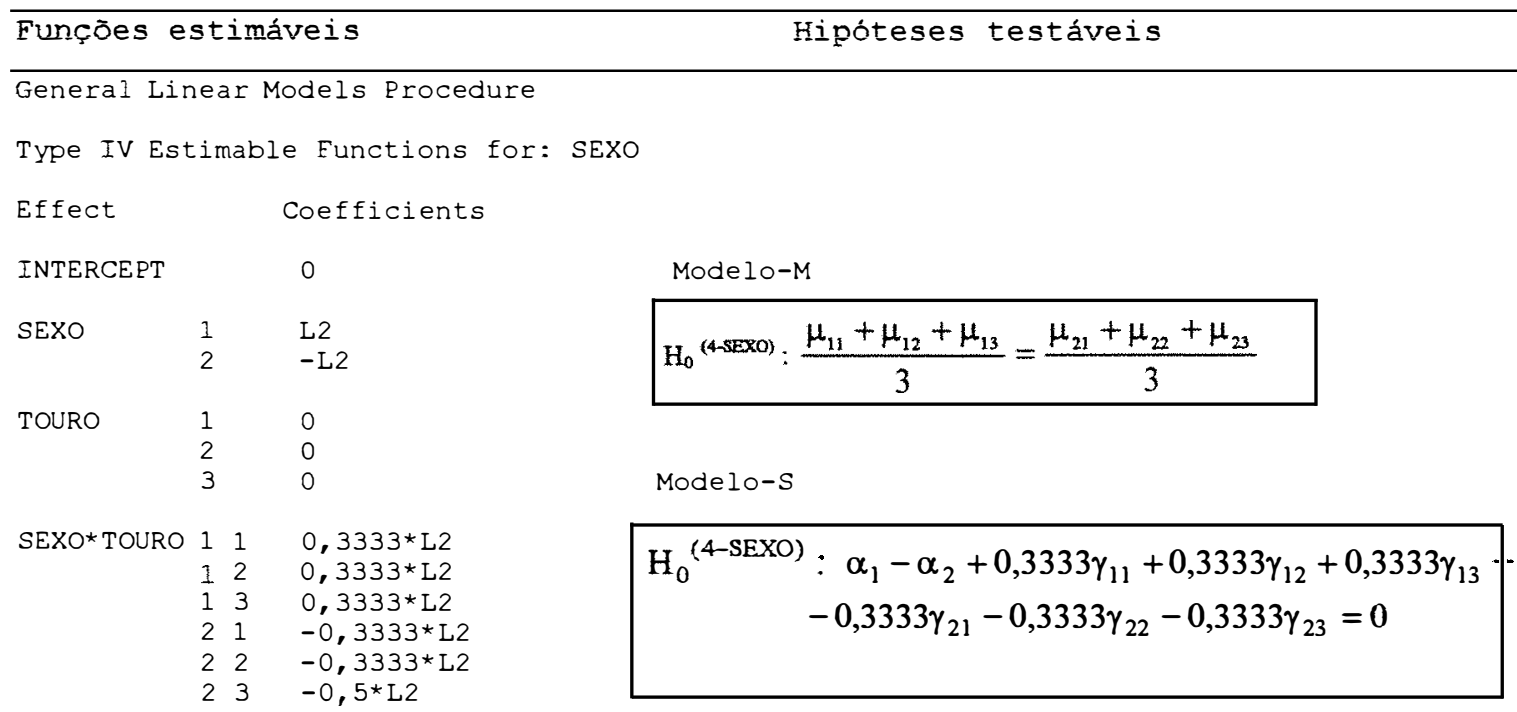

Type IV Estimable Eunctions for: TOURO

\begin{tabular}{|c|c|c|c|c|}
\hline Effect & & Defficients & Modelo-M & \\
\hline INTERCEPT & & 0 & & $\mu_{11}+\mu_{21}-\mu_{13}+\mu_{23}$ \\
\hline SEXO & 1 & 0 & (4-TOURO). & $2=\frac{2}{2}$ \\
\hline & 2 & 0 & $\Lambda_{0}$ & $\mu_{12}+\mu_{22}-\mu_{13}+\mu_{23}$ \\
\hline TOURO & $\begin{array}{l}1 \\
2\end{array}$ & $\begin{array}{l}\text { L4 } \\
\text { L5 }\end{array}$ & & 2 \\
\hline & 3 & $-L 4-L 5$ & Modelo-s & \\
\hline SEXO*TOURO & $\begin{array}{ll}1 & 1 \\
1 & 2 \\
1 & 3 \\
2 & 1 \\
2 & 2\end{array}$ & $\begin{array}{l}0,5 * L 4 \\
0,5 * L 5 \\
-0,5 * L 4-0,5 * L 5 \\
0,5 * L 4 \\
0,5 * L 5\end{array}$ & $\begin{array}{l}\mathrm{H}_{0}^{\text {(4-TOURO) }}: \\
\left\{\begin{array}{l}\beta_{1}-\beta_{3}+0,5 \gamma_{11}- \\
\beta_{2}-\beta_{3}+0,5 \gamma_{12}-\end{array}\right.\end{array}$ & $\begin{array}{l}0,5 \gamma_{13}+0,5 \gamma_{21}-0,5 \gamma_{23}=0 \\
0,5 \gamma_{13}+0,5 \gamma_{22}-0,5 \gamma_{23}=0\end{array}$ \\
\hline
\end{tabular}

Type IV Estimable Eunctions for: SEXO*TOURO

Effect Coefficients

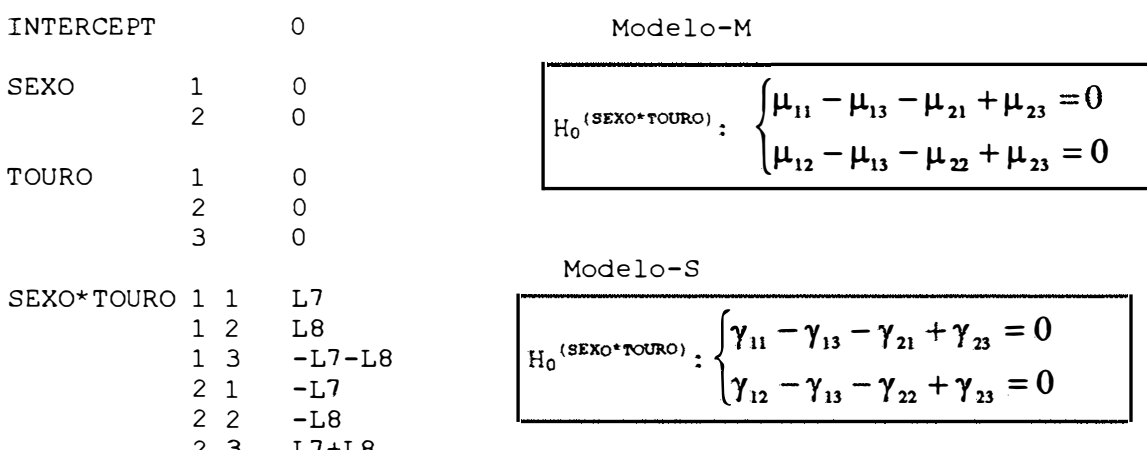


Observa-se na Tabela 10, conforme já comentado em 4.3.1, que as hipóteses do tipo IV são similares as tipos do tipo III, pois não existe casela vazia no conjunto de dados apresentado na Tabela 3.

\subsubsection{Análise de variância}

A Tabela 11 apresenta os resultados obtidos através da análise de variância do PROC GLM segundo a especificação MODEL, e as somas de quadrados dos tipos I, II, III e IV respectivamente.

O PROC GLM considera todos os efeitos como fixos, logo o denominador dos testes "F" está baseado no quadrado médio do resíduo do modelo. e portanto todos esses testes não devem ser considerados, no caso do modelo misto.

Os testes na Tabela 11, são feitos com o resíduo e são testes exatos para modelos fixos. Esses testes, no entanto, não são apropriados para o modelo misto, pois eles estão considerando todos os efeitos como fixos.

As hipóteses que estão sendo testadas na Tabela 11 , e as respectivas somas de quadrados descritas por meio da notação $\mathrm{R}(\cdot)$, estão destacadas no retângulo, e estão descritas nas Tabelas de 7 a 10.

Verifica-se, também, na Tabela 11, conforme esperado e já comentado em 4.3.1, pois não há caselas vazias, que as somas de quadrados dos tipos III e IV são as mesmas. Além disso, dado que a interação está ajustada para todos os demais efeitos, as quatro somas de quadrados a ela relacionadas são iguais.

Os testes dos tipos I e II para touro são iguais, pois conforme já comentado em 5.1.1.2, as funções estimáveis do tipo I para touro são na realidade do tipo II, o que pode ser observado pela notação $\mathrm{R}(\cdot)$ em destaque no retângulo. 
Tabela 11. Análise de variância do procedimento GLM, e as suas respectivas hipóteses.

Dependent Variable: p365

$$
\text { General Linear Models Procedure }
$$

$\begin{array}{lrrrrr}\text { Source } & \text { DF } & \text { Sum of Squares } & \text { Mean Square } & \text { F Value } & \text { Pr }>\text { F } \\ \text { Model } & 5 & 20587,040909 & 4117,408182 & 8,73 & 0,0004 \\ \text { Error } & 16 & 7545,550000 & 471,596875 & & \\ \text { Corrected Total } & 21 & 28132,590909 & & \end{array}$

\begin{tabular}{|c|c|}
\hline & $\begin{array}{l}\text { R-Square } \\
0,731786\end{array}$ \\
\hline $\mathrm{H}_{0}$ & $\mathrm{R}(\cdot)$ \\
\hline $\mathrm{H}_{0}^{(1-\text { SEXo) }}$ & $\mathrm{R}(\boldsymbol{\alpha} \mid \mu)$ \\
\hline $\mathrm{H}_{0}{ }^{(2-\text { TOURO })}$ & $R(\beta \mid \mu, \alpha)$ \\
\hline $\mathrm{H}_{0}{ }^{\text {(SEXOTOURO) }}$ & $\mathrm{R}(\gamma \mid \mu, \alpha, \beta)$ \\
\hline $\mathrm{H}_{\mathrm{o}}^{(2-\operatorname{sexO})}$ & $\mathrm{R}(\alpha \mid \mu, \beta)$ \\
\hline $\mathrm{H}_{0}{ }^{(2-\text { TOURO })}$ & $R(\beta \mid \mu, \alpha)$ \\
\hline $\mathrm{H}_{0}$ (SEXOTOURO) & $\mathrm{R}(\gamma \mid \mu, \alpha, \beta)$ \\
\hline $\mathrm{H}_{0}{ }^{(3 \operatorname{SEXXO})}$ & $\mathrm{R}(\alpha \mid \mu, \beta, \gamma)$ \\
\hline $\mathrm{H}_{0}{ }^{(3-\mathrm{TOURO})}$ & $R(\beta \mid \mu, \alpha, \gamma)$ \\
\hline $\mathrm{H}_{0}{ }^{\text {(SEXOTOURO) }}$ & $\mathrm{R}(\gamma \mid \mu, \alpha, \beta)$ \\
\hline $\mathrm{H}_{0}{ }^{(4 \text { SExo })}$ & $\mathrm{R}(\alpha \mid \mu, \beta, \gamma)$ \\
\hline $\mathrm{H}_{0}{ }^{\text {(4-TOURO })}$ & $R(\beta \mid \mu, \alpha, \gamma)$ \\
\hline $\mathrm{H}_{\mathrm{o}}$ (SEXOTOURO) & $\mathrm{R}(\gamma \mid \mu, \alpha, \beta)$ \\
\hline
\end{tabular}

\begin{tabular}{|c|c|c|c|c|c|}
\hline \multirow[b]{2}{*}{ Source } & \multicolumn{2}{|c|}{$\begin{array}{r}\text { C.V. } \\
10,10701\end{array}$} & $\begin{array}{l}\text { Root MSE } \\
21,716281\end{array}$ & \multicolumn{2}{|c|}{$\begin{array}{r}\text { Y Mean } \\
214,86364\end{array}$} \\
\hline & $\mathrm{DF}$ & Type I SS & Mean Square & F Value & $\operatorname{Pr}>\mathrm{F}$ \\
\hline $\begin{array}{l}\text { SEXO } \\
\text { TOURO } \\
\text { SEXO* TOURO }\end{array}$ & $\begin{array}{l}1 \\
2 \\
2\end{array}$ & $\begin{array}{r}15838,471251 \\
3220,397771 \\
1528,171888\end{array}$ & $\begin{array}{r}15838,471251 \\
1610,198885 \\
764,085944\end{array}$ & $\begin{array}{c}33,58 \\
3,41 \\
1,62\end{array}$ & $\begin{array}{l}0,0001 \\
0,0582 \\
0,2287\end{array}$ \\
\hline Source & $\mathrm{DF}$ & Type II SS & Mean Square & F Value & $\operatorname{Pr}>\mathrm{F}$ \\
\hline $\begin{array}{l}\text { SEXO } \\
\text { TOURO } \\
\text { SEXO*TOURO }\end{array}$ & $\begin{array}{l}1 \\
2 \\
2\end{array}$ & $\begin{array}{r}14636,881287 \\
3220,397771 \\
1528,171888\end{array}$ & $\begin{array}{r}14636,881287 \\
1610,198885 \\
764,085944\end{array}$ & $\begin{array}{r}31,04 \\
3,41 \\
1,62\end{array}$ & $\begin{array}{l}0,0001 \\
0,0582 \\
0,2287\end{array}$ \\
\hline Source & $\mathrm{DF}$ & Type III SS & Mean Square & F Value & $\mathrm{Pr}>\mathrm{F}$ \\
\hline $\begin{array}{l}\text { SEXO } \\
\text { TOURO } \\
\text { SEXO* TOURO }\end{array}$ & $\begin{array}{l}1 \\
2 \\
2\end{array}$ & $\begin{array}{r}13463,272430 \\
3324,838554 \\
1528,171888\end{array}$ & $\begin{array}{r}13463,272430 \\
1662,419277 \\
764,085944\end{array}$ & $\begin{array}{r}28,55 \\
3,53 \\
1,62\end{array}$ & $\begin{array}{l}0,0001 \\
0,0539 \\
0,2287\end{array}$ \\
\hline Source & $\mathrm{DF}$ & Type IV sS & Mean Square & F Value & $\operatorname{Pr}>\mathrm{F}$ \\
\hline $\begin{array}{l}\text { SEXO } \\
\text { TOURO } \\
\text { SEXO TOURO }\end{array}$ & $\begin{array}{l}1 \\
2 \\
2\end{array}$ & $\begin{array}{r}13463,272430 \\
3324,838554 \\
1528,171888\end{array}$ & $\begin{array}{r}13463,272430 \\
1662,419277 \\
764,085944\end{array}$ & $\begin{array}{r}28, \\
3, \\
1,\end{array}$ & $\begin{array}{l}0,0001 \\
0,0539 \\
0,2287\end{array}$ \\
\hline
\end{tabular}

\subsubsection{Declaração RANDOM / TEST}

A declaração RANDOM especifica que os fatores touro e sexo*touro são aleatórios e fornece os quadrados médios esperados para todos os efeitos considerados no modelo. Como default fornece os quadrados médios esperados do tipo III. Se forem 
utilizadas as opções E1, E2 e E4 depois do MODEL, o RANDOM fornece os quadrados médios esperados dos tipos I, II e IV, respectivamente.

Analogamente, a opção TEST pode fornecer os quatro tipos de testes para cada efeito do modelo e os quadrados médios esperados apropriados como termos do erro. Esses são os testes que devem ser considerados no caso do modelo misto.

Tabela 12. Quadrados médios esperados e os respectivos testes para o tipo I.

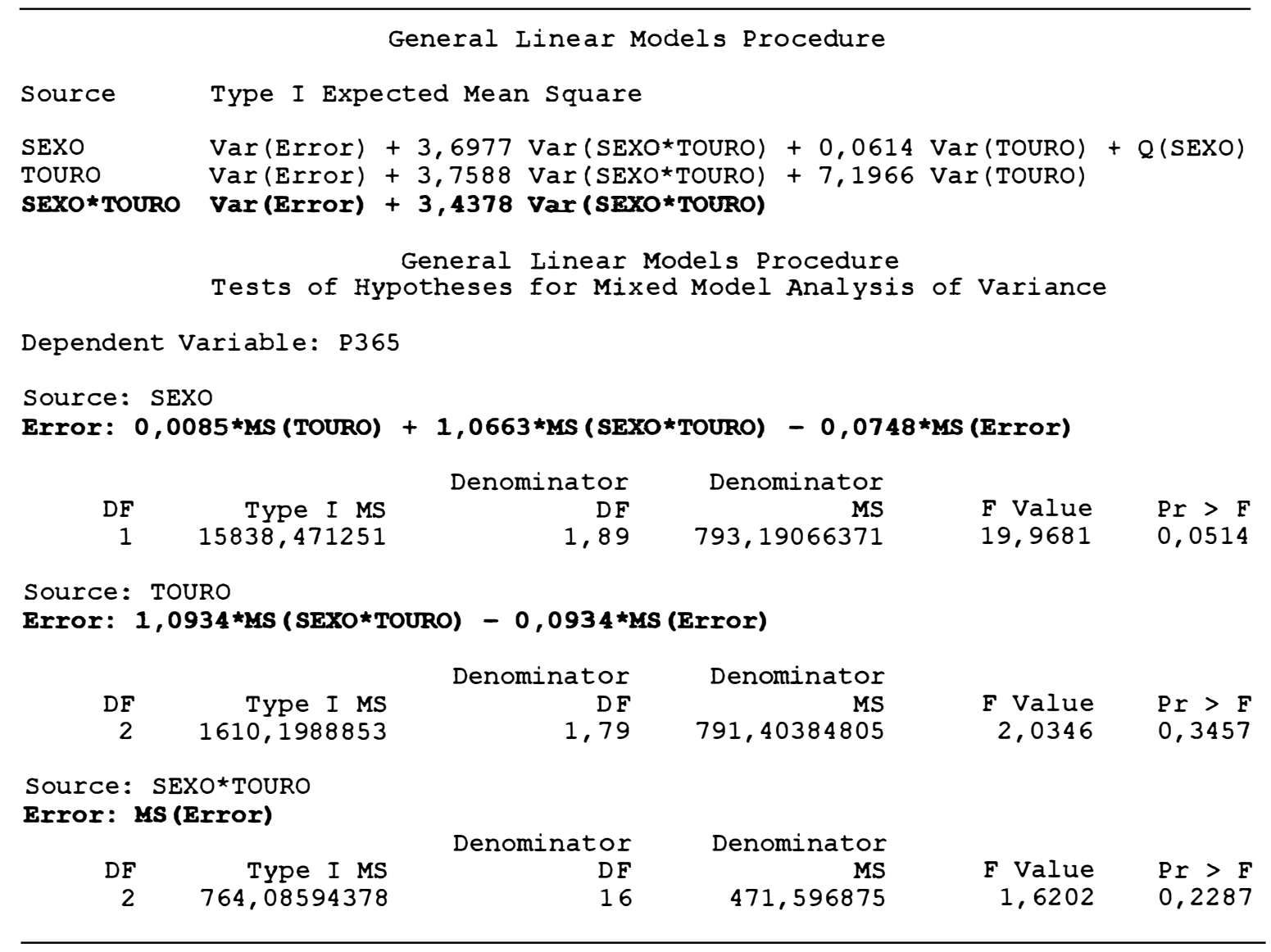

Observa-se na Tabela 12, que a soma de quadrados do tipo I para o fator de efeito fixo, sexo, é a mesma encontrada na Tabela 11. No entanto, os quadrados médios, os graus de liberdade e o valor de F são diferentes. Ou seja, está sendo testada a mesma hipótese $\mathrm{H}_{0}^{(1-S e x o)}$ mas, levando-se em consideração que os efeitos de touro e da interação 
sexo*touro são aleatórios. Isto ocorre, pois o quadrado médio do denominador está corrigido conforme já colocado no item 4.2.4.

Na Tabela 12, tem-se as seguintes esperanças dos quadrados médios:

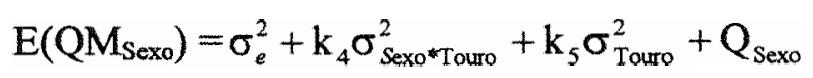

onde $\mathrm{Q}_{\text {sexo }}=0$ se e somente se $\mathrm{H}_{0}$ é verdadeira.

$$
\begin{aligned}
& \mathrm{E}\left(\mathrm{QM}_{\text {Touro }}\right)=\sigma_{e}^{2}+\mathrm{k}_{3} \sigma_{\text {Sexo*Touro }}^{2}+\mathrm{k}_{2} \sigma_{\text {Touro }}^{2} \\
& \mathrm{E}\left(\mathrm{QM}_{\text {sexo*Touro }}\right)=\sigma_{e}^{2}+\mathrm{k}_{1} \sigma_{\text {Sexo*Touro }}^{2} \\
& \mathrm{E}\left(\mathrm{QM}_{\mathrm{Erro}}\right)=\sigma_{e}^{2}
\end{aligned}
$$

onde: $k_{1}=3,4378 ; k_{2}=7,1966 ; k_{3}=3,7588 ; k_{4}=3,6977 ; k_{5}=0,0614$

Logo, para o fator fixo, sexo, o quadrado médio do denominador corrigido, é

$$
\mathrm{QM}^{*}=\mathrm{a}_{1} \mathrm{QM}_{\mathrm{Emo}}+\mathrm{a}_{2} \mathrm{QM}_{\text {Sexo*Touro }}+\mathrm{a}_{3} \mathrm{QM}_{\text {Touro }}
$$

onde $a_{1}=1-\frac{k_{5}}{k_{2}}-\frac{k_{4}}{k_{1}}+\frac{k_{3} k_{5}}{k_{1} k_{2}}=1-\frac{0,06143,6977}{7,1966} 3,4378+\frac{(3,7588)(0,0614)}{(3,4378)(7,1966)}=-0,0748$

$$
\begin{aligned}
& a_{2}=\frac{k_{4}}{k_{1}}-\frac{k_{3} k_{5}}{k_{1} k_{2}}=\frac{3,6977}{3,4378}-\frac{(3,7588)(0,0614)}{(3,4378)(7,1966)}=1,0663 \\
& a_{3}=\frac{k_{5}}{k_{2}}=\frac{0,0614}{7,1966}=0,0085
\end{aligned}
$$

e $\mathrm{QM}_{\mathrm{Emo}}=471,5969 ; \mathrm{QM}_{\text {Sexo }}{ }_{\text {Touro }}=764,0859 ; \mathrm{QM}_{\text {Touro }}=1610,1989$

$$
\mathrm{QM}^{*}=(-0,0748)(471,5969)+(1,0663)(764,0859)+(0,0085)(1610,1989)=793,19
$$

Portanto, o teste estatístico, para o efeito fixo, sexo, é:

$$
\mathrm{F}=\frac{\mathrm{QM}_{\text {Sexo }}}{\mathrm{QM}^{*}}=\frac{15838,47}{793,19}=19,9681
$$

o qual tem distribuição aproximada F com 1 e d graus de liberdade, onde d é calculado pelo método de Satterthwaite por

$$
\mathrm{d}=\frac{\left(\mathrm{QM}^{*}\right)^{2}}{\frac{\left(\mathrm{a}_{1} \mathrm{QM}_{\mathrm{ERO}}\right)^{2}}{\mathrm{gl}_{\mathrm{QM}_{\mathrm{ERO}}}}+\frac{\left(\mathrm{a}_{2} \mathrm{QM}_{\text {Sexo }} \mathrm{Touro}\right)^{2}}{\mathrm{gl}_{\mathrm{QM}_{\text {Sexo Touro }}}}+\frac{\left(\mathrm{a}_{3} \mathrm{QM}_{\text {Touro }}\right)^{2}}{\mathrm{gl}_{\mathrm{QM}_{\text {Touro }}}}}
$$




$$
d=\frac{(793,19)^{2}}{\frac{[(-0,0748)(471,5969)]^{2}}{16}+\frac{[(1,0663)(764,0859)]^{2}}{2}+\frac{[(0,0085)(1610,1989)]^{2}}{2}}=1,89
$$

A Tabela 12 fornece os erros adequados para os testes com os respectivos graus de liberdade calculados pelo método de Satterthwaite.

$\mathrm{Na}$ Tabela 12 não há testes exatos para os efeitos principais, isso pode ser verificado quando se observa as esperanças dos quadrados médios. $O$ teste é exato apenas para a interação.

Os testes dos efeitos principais, aqui, são feitos com a interação e o teste da interação com o resíduo.

Tabela 12a. Nível nominal dos testes do tipo I

\begin{tabular}{ccc}
\hline \multirow{2}{*}{ Causa de Variação } & \multicolumn{2}{c}{ Nível nominal do teste (Pr > F) } \\
\cline { 2 - 3 } & Modelo Fixo (Tabela 11) & Modelo Misto (Tabela 12) \\
\hline Sexo & 0,0001 & 0,0514 \\
Touro & 0,0582 & 0,3457 \\
Sexo*Touro & 0,2287 & 0,2287 \\
\hline
\end{tabular}

Comparando os testes da Tabela 12 com os testes do tipo I da Tabela 11, quanto ao nível nominal do teste, observa-se, na Tabela 12a, que para o efeito fixo, sexo, o valor que era altamente significativo agora é apenas significativo. Para touros houve uma drástica mudança do nível nominal do teste e para interação não há mudança no nível conforme era esperado, pois no caso da interação o teste é feito com o resíduo, em ambos os modelos.

Na Tabela 13 tem-se os quadrados médios esperados e os respectivos testes de hipóteses para o tipo II.

Observa-se na Tabela 13, que a soma de quadrados do tipo II para o fator fixo, sexo, é a mesma da Tabela 11, mas os quadrados médios, os graus de liberdade e o valor F são diferentes. 
Tabela 13. Quadrados médios esperados e os respectivos testes de hipóteses do tipo II.

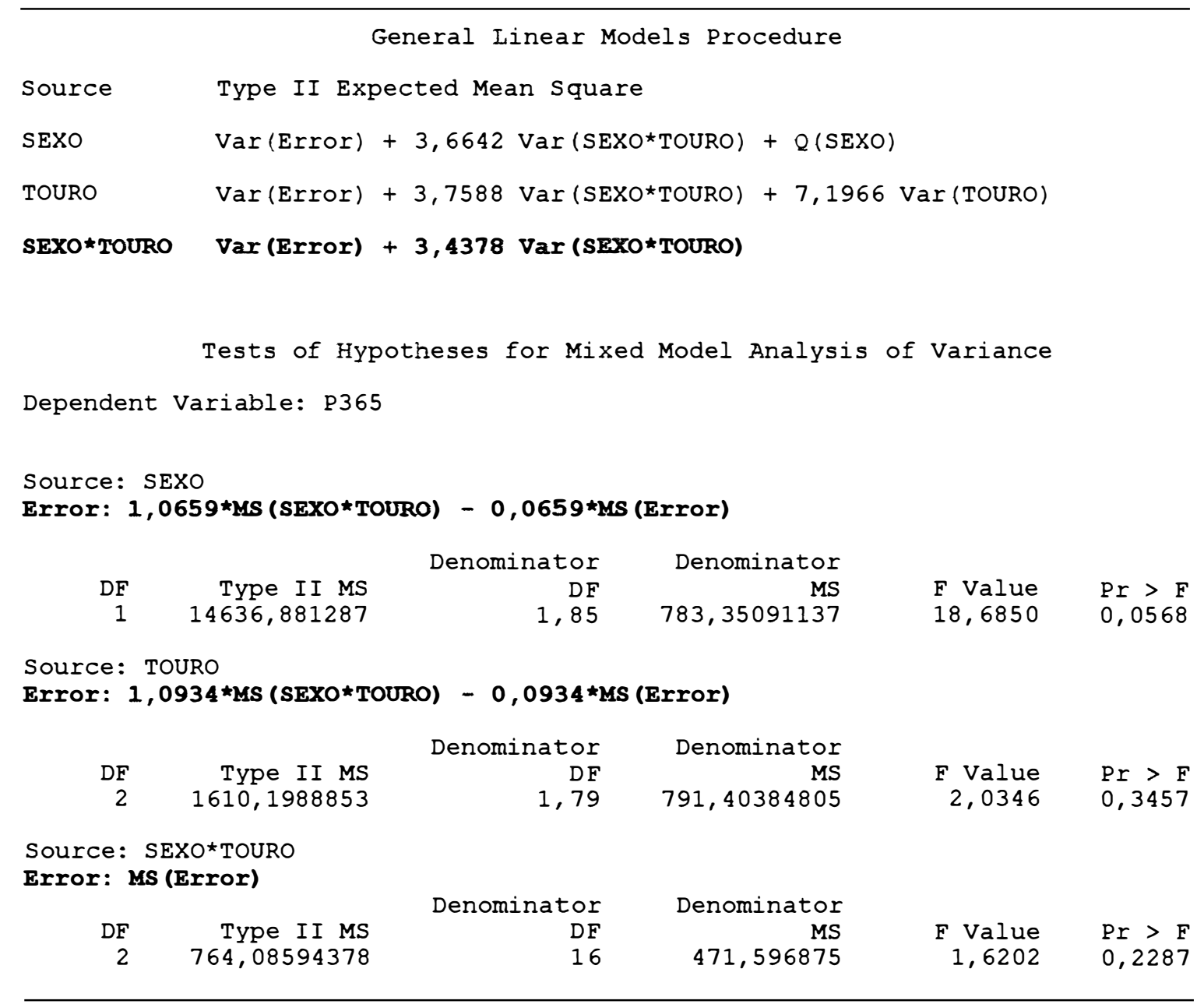

Comparando os valores da Tabela 13 com os da Tabela 12, observa-se que os testes para a interação e para touro são os mesmos, conforme já comentado em 5.1.1.2, pois a interação está ajustada para todos os demais efeitos e o teste do tipo I para touro é, na realidade, do tipo II.

Na Tabela 13, exceto para a interação, os testes para os efeitos principais não são exatos e os graus de liberdade são calculados pelo método de Satterthwaite.

A Tabela 13 fornece os erros adequados para os testes do tipo II, enquanto que os testes para o tipo II da Tabela 11 não são apropriados. 
Tabela 13a. Nível nominal dos testes do tipo II

\begin{tabular}{|c|c|c|}
\hline \multirow{2}{*}{ Causa de Variação } & \multicolumn{2}{|c|}{ Nível nominal do teste $(\operatorname{Pr}>\mathrm{F})$} \\
\hline & Modelo Fixo (Tabela 11) & Modelo Misto (Tabela 13) \\
\hline Sexo & 0,0001 & 0,0568 \\
\hline Touro & 0,0582 & 0,3457 \\
\hline Sexo*Touro & 0,2287 & 0,2287 \\
\hline
\end{tabular}

$\mathrm{Na}$ Tabela 13a tem-se o nível nominal dos testes das hipóteses do tipo II, onde se observa que o nível nominal praticamente não se altera, em relação aos testes das hipóteses do tipo I, Tabela 12a.

Tabela 14. Quadrados médios esperados e os respectivos testes de hipóteses do tipo III.

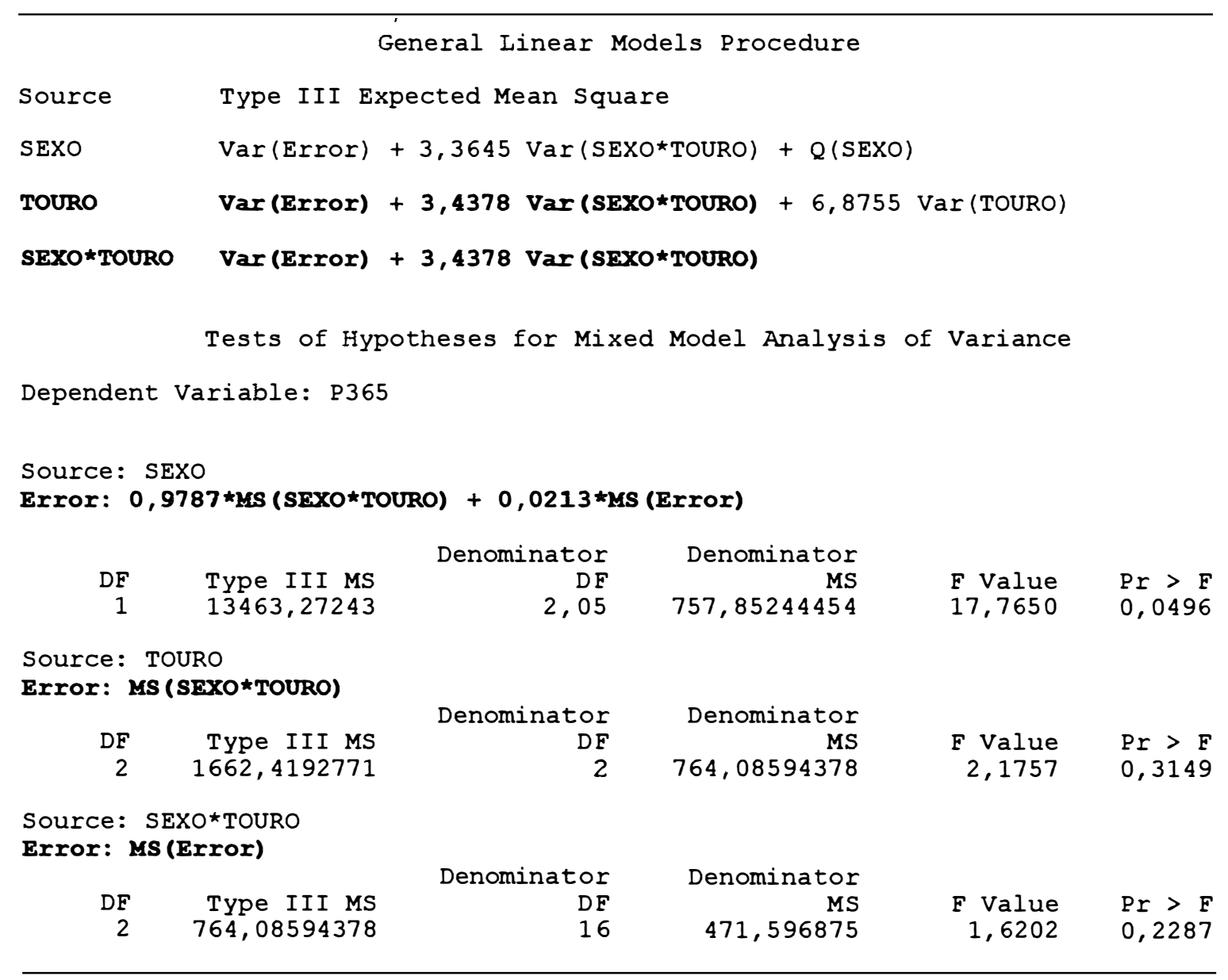


Observa-se também na Tabela 14, como nas Tabelas 12 e 13, que as correspondentes somas de quadrados, aqui do tipo III para o fator fixo, sexo, são as mesmas da Tabela 11, mas os quadrados médios, os graus de liberdade e o valor $F$ são diferentes.

Nas Tabelas 12 e 13, os testes dos tipos I e II respectivamente, para touro não são testes exatos. Agora o teste tipo III apresentado na Tabela 14 é exato, como pode ser observado através das esperanças dos quadrados médios.

Na Tabela 14a, tem-se o nível nominal dos testes do tipo III para as Tabelas $11 \mathrm{e}$ 14.

Tabela 14a. Nível nominal dos testes do tipo III

\begin{tabular}{ccc}
\hline \multirow{2}{*}{ Causa de Variação } & \multicolumn{2}{c}{ Nível nominal do teste (Pr $>$ F) } \\
\cline { 2 - 3 } & Modelo Fixo (Tabela 11) & Modelo Misto (Tabela 14) \\
\hline Sexo & 0,0001 & 0,0496 \\
Touro & 0,0539 & 0,3149 \\
Sexo*Touro & 0,2287 & 0,2287 \\
\hline
\end{tabular}

$\mathrm{Na}$ Tabela 14a tem-se o nível nominal dos testes das hipóteses do tipo III, onde se observa que o nível nominal praticamente não se altera, em relação aos testes das hipóteses dos tipos I e II, Tabelas 12a e 13a.

A Tabela 15 é similar à Tabela 14, pois como já comentado, não existem caselas vazias e nesse caso e as hipóteses dos tipos III e IV são equivalentes. Além disso, as somas de quadrados a elas associadas são ajustadas para todos os outros fatores. 
Tabela 15. Quadrados médios esperados e os respectivos testes de hipóteses do tipo IV.

\begin{tabular}{|c|c|c|c|c|c|}
\hline \multicolumn{6}{|c|}{ General Linear Models Procedure } \\
\hline $\begin{array}{l}\text { SEXO } \\
\text { TOURO } \\
\text { SEXO*TOURO }\end{array}$ & \multicolumn{4}{|c|}{$\begin{array}{l}\operatorname{Var}(\text { Error) }+3,3645 \operatorname{Var}(\text { SEXO*TOURO) }+Q(\text { SEXO) } \\
\operatorname{Var} \text { (Error) }+3,4378 \operatorname{Var}(\text { SEXO*TOURO) }+6,8755 \operatorname{Var}(\text { TOURO) } \\
\operatorname{Var} \text { (Error) }+3,4378 \operatorname{Var}(\text { SEXO*TOURO) }\end{array}$} & \\
\hline \multicolumn{6}{|c|}{ Tests of Hypotheses for Mixed Model Analysis of Variance } \\
\hline \multicolumn{6}{|c|}{$\begin{array}{l}\text { Source: SEXO } \\
\text { Error: } 0,9787 \star M S(S E X O \star T O U R O)+0,0213 \star M S \text { (Error) }\end{array}$} \\
\hline $\begin{array}{r}\mathrm{DF} \\
1\end{array}$ & $\begin{array}{l}\text { Type IV MS } \\
13463,27243\end{array}$ & $\begin{array}{r}\text { Denominator } \\
\text { DF } \\
2,05\end{array}$ & $\begin{array}{r}\text { Denominator } \\
\text { MS } \\
757,85244454\end{array}$ & $\begin{array}{l}\text { F Value } \\
17,7650\end{array}$ & $\begin{array}{l}\text { Pr }>F \\
0,0496\end{array}$ \\
\hline \multicolumn{6}{|c|}{$\begin{array}{l}\text { Source: TOURO } \\
\text { Error: MS (SEXO^TOURO) }\end{array}$} \\
\hline $\begin{array}{r}\mathrm{DF} \\
2\end{array}$ & $\begin{array}{l}\text { Type IV MS } \\
1662,4192771\end{array}$ & $\begin{array}{r}\text { Denominator } \\
\text { DF } \\
2\end{array}$ & $\begin{array}{r}\text { Denominator } \\
\text { MS } \\
764,08594378\end{array}$ & $\begin{array}{l}\text { F Value } \\
2,1757\end{array}$ & $\begin{array}{l}\operatorname{Pr}>F \\
0,3149\end{array}$ \\
\hline \multicolumn{6}{|c|}{$\begin{array}{l}\text { Source: SEXO*TOURO } \\
\text { Error: MS (Error) }\end{array}$} \\
\hline $\begin{array}{r}\mathrm{DF} \\
2\end{array}$ & $\begin{array}{l}\text { Type IV MS } \\
764,08594378\end{array}$ & $\begin{array}{r}\text { Denominator } \\
\text { DF } \\
16\end{array}$ & $\begin{array}{r}\text { Denominator } \\
\text { MS } \\
471,596875\end{array}$ & $\begin{array}{r}\text { F Value } \\
1,6202\end{array}$ & $\begin{array}{l}\operatorname{Pr}>F \\
0,2287\end{array}$ \\
\hline
\end{tabular}

\subsubsection{Procedimento VARCOMP}

O PROC VARCOMP fornece as estimativas para os componentes de variância por meio de quatro métodos de estimação: tipo I (ANOVA baseado no método III de Henderson), MIVQUE0, ML e REML. 


\subsubsection{Um programa}

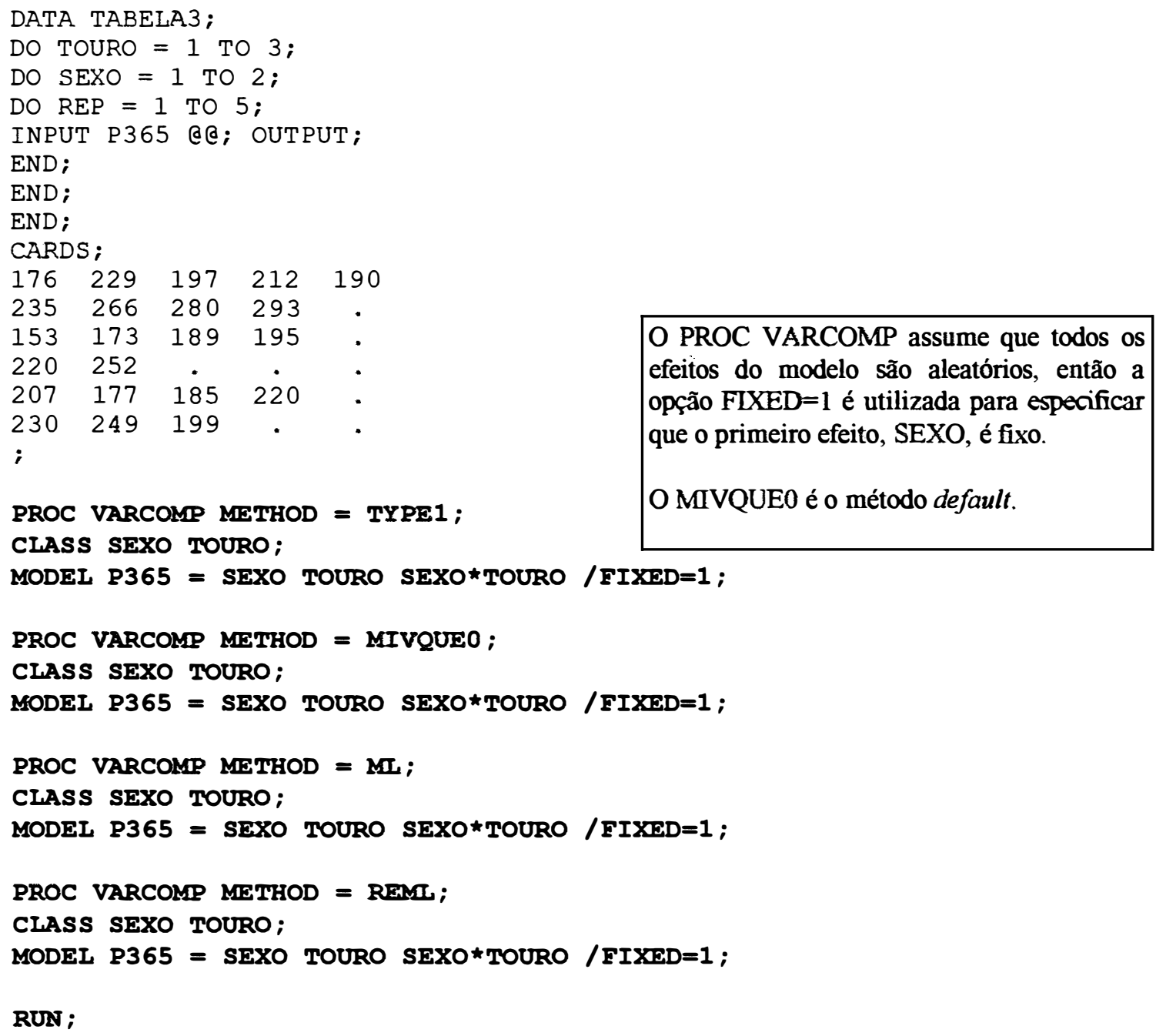

\subsubsection{Método ANOVA}

Este método (METHOD=TYPE1) calcula as somas de quadrados do tipo I para cada efeito e fornece as estimativas do tipo I ( método III de Henderson) para cada efeito 
aleatório. Este método pode fornecer estimativas negativas para os componentes de variância.

Tabela 16. Resultados obtidos através do PROC VARCOMP quando se utiliza a opção METHOD=TYPE 1 .

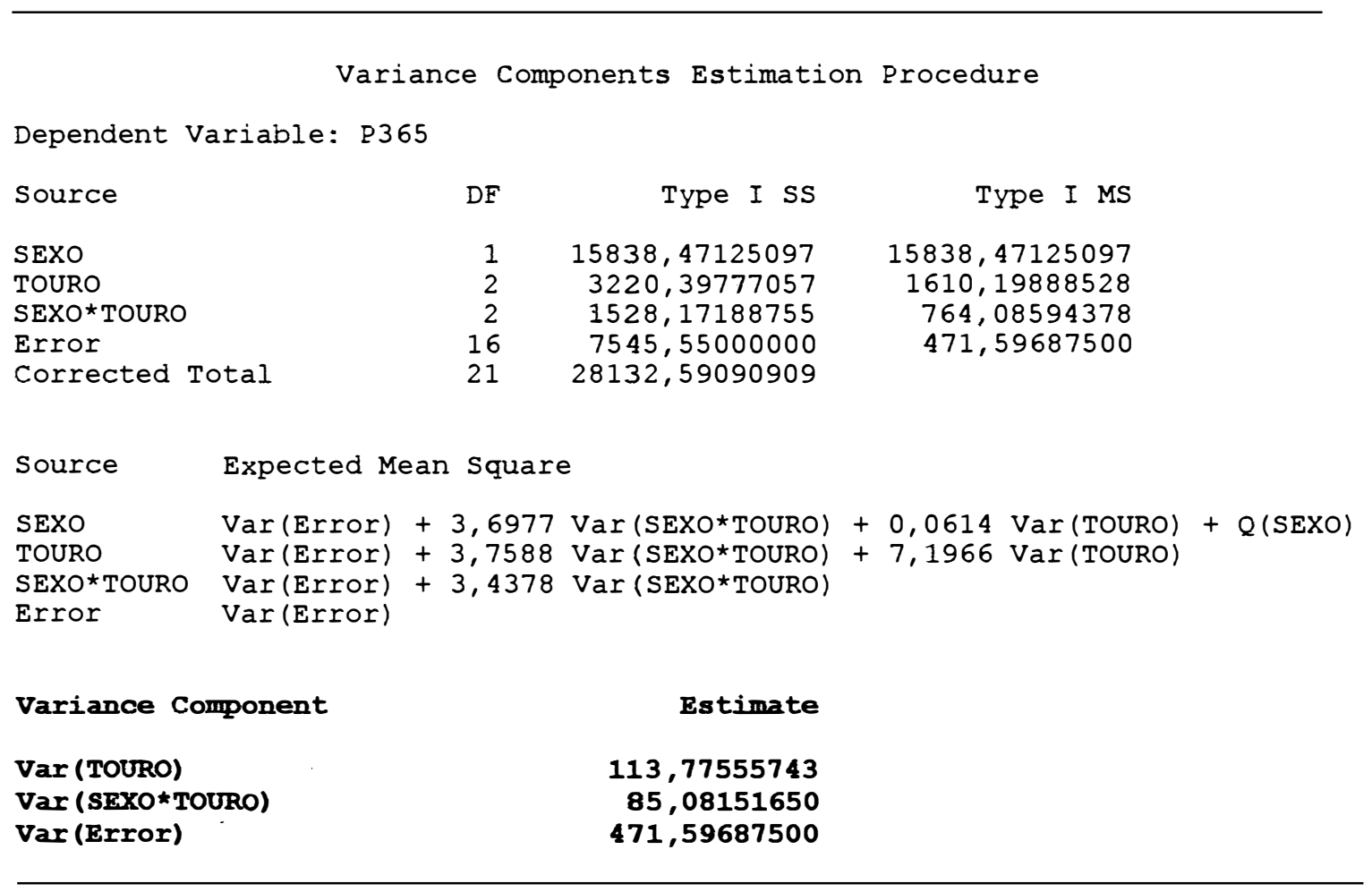

Observa-se na Tabela 16 as mesmas expressões dos quadrados médios esperados obtidos na Tabela 12 e as mesmas somas de quadrados do tipo I, da Tabela 11. As estimativas dos componentes de variância são dadas por:

$$
\hat{\sigma}_{\text {touro }}^{2}=113,78 \quad \hat{\sigma}_{\text {sexortouro }}^{2}=85,08 \quad \hat{\sigma}_{e}^{2}=471,60
$$

Pela primeira vez, desde a Tabela 11, tem-se as estimativas dos componentes de variância conforme Searle (1987), Searle et al. (1992), e como discutido em 4.2.2.4, as estimativas obtidas pelo método III de Henderson são não viesadas. 


\subsubsection{Método MIVQUE0}

O método MIVQUE0 fornece estimativas para os componentes de variância que são não viesadas, invariantes e de variância mínima (Searle, 1987).

Tabela 17. Resultados obtidos através do PROC VARCOMP quando se utiliza a opção METHOD=MIVQUE0.

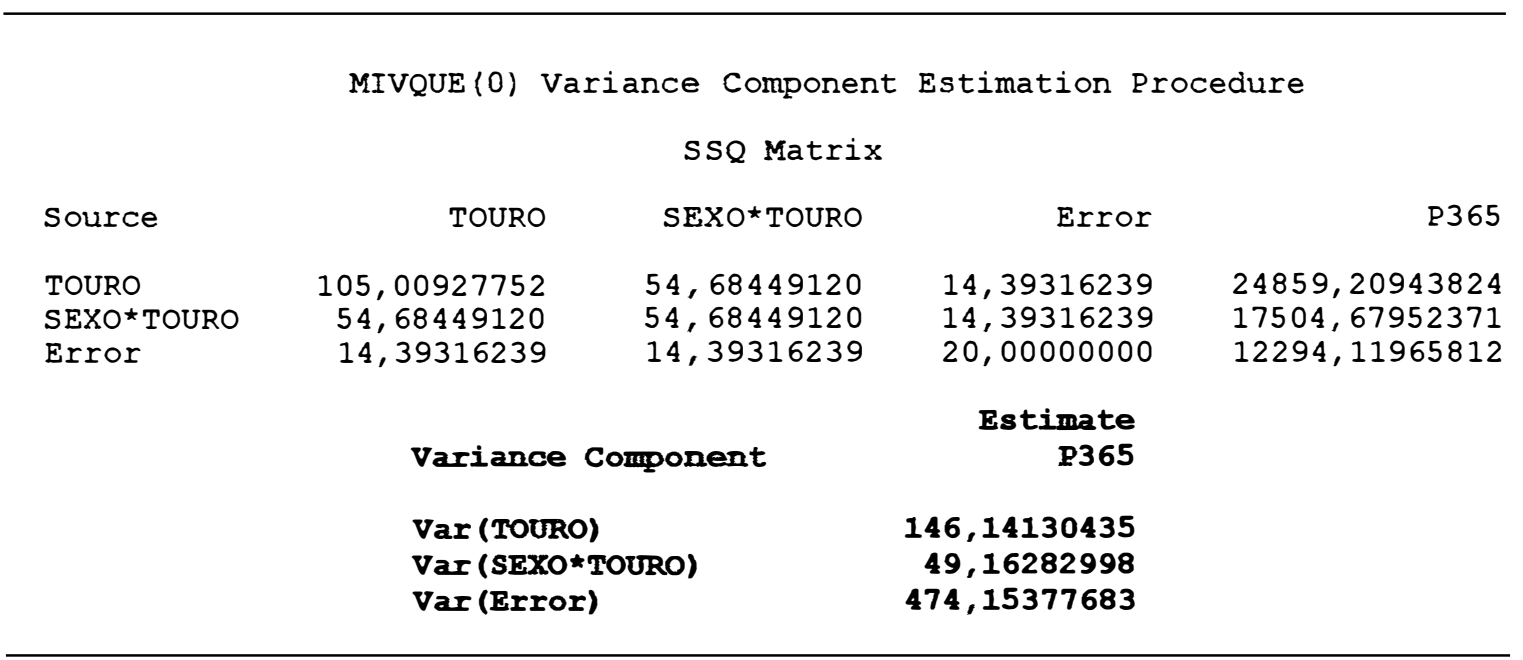

A Tabela 17 fornece a matriz SSQ, que é a matriz cujo elemento $(i, j)$ é dado por $\left\{\operatorname{tr}\left(\mathbf{P V}_{\mathbf{i}} \mathbf{P} \mathbf{V}_{\mathbf{j}}\right)\right\}$ de acordo com o lado esquerdo da equação (27), e também as estimativas dos componentes de variância conforme (27). Assim tem-se as estimativas pelo método MIVQUE0:

$$
\hat{\sigma}_{\text {touro }}^{2}=146,14 \quad \hat{\sigma}_{\text {sexo*touro }}^{2}=49,16 \quad \hat{\sigma}_{e}^{2}=474,15
$$

Aqui tem-se novas estimativas, dos componentes de variância, diferentes daquelas obtidas na Tabela 16. Não há, em situações práticas, como nas tabelas seguintes, como saber, exatamente, qual a melhor estimativa. Um critério de escolha, recomendado na literatura, refere-se à adequação aos dados, do método adotado.

As estimativas obtidas pelo método MIVQUE0 são não viesadas, invariantes e de variância mínima, conforme visto em 4.2.2.8. 


\subsubsection{Método ML}

O método da máxima verossimilhança é um método iterativo e fornece sempre estimativas não-negativas para os componentes de variância.

Tabela 18. Resultados obtidos através do PROC VARCOMP quando se utiliza a opção METHOD=ML.

Maximum Likelihood Variance Components Estimation Procedure

Dependent Variable: P365

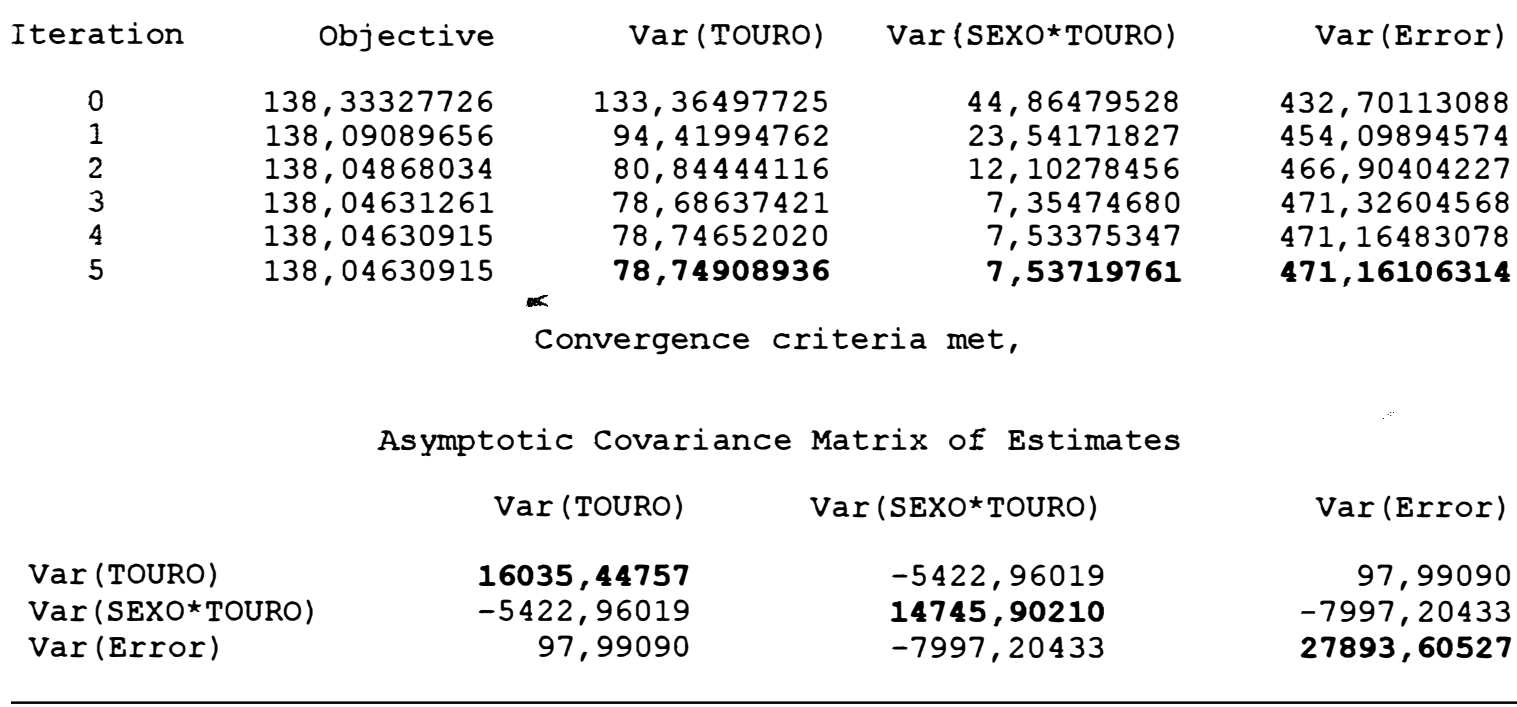

Observa-se na Tabela 18 que o processo numérico para obter as estimativas $\mathrm{ML}$ convergiu na $5^{\mathbf{a}}$ iteração, fornecendo as estimativas dos componentes de variância:

$$
\hat{\sigma}_{\text {touro }}^{2}=78,75 \quad \hat{\sigma}_{\text {sexortouro }}^{2}=7,54 \quad \hat{\sigma}_{e}^{2}=471,16
$$

As estimativas ML, conforme visto em 4.2.2.5 são viesadas. Com exceção de $\hat{\sigma}_{e}^{2}$, verifica-se que as demais estimativas aqui obtidas são diferentes daquelas obtidas na Tabela 16. 
Utilizando-se o método ML, o PROC VARCOMP fornece além das estimativas dos componentes de variância a matriz de covariâncias assintóticas.

As variâncias assintóticas das estimativas dos componentes de variância são dadas conforme a Tabela 18, por:

$$
\operatorname{Var}\left(\hat{\sigma}_{\text {touro }}^{2}\right)=16035,45 \quad \operatorname{Var}\left(\hat{\sigma}_{\text {sexo tauro }}^{2}\right)=14745,90 \quad \operatorname{Var}\left(\hat{\sigma}_{e}^{2}\right)=27893,61
$$

\subsubsection{Método REML}

O método da máxima verossimilhança restrita é, também, um método iterativo e fornece estimativas não-negativas para os componentes de variância.

Tabela 19. Resultados obtidos através do PROC VARCOMP quando se utiliza a opção METHOD=REML.

Restricted Maximum Likelihood Variance Components Estimation Procedure Dependent Variable: P365

\begin{tabular}{|c|c|c|c|c|}
\hline Iteration & Objective & $\operatorname{Var}($ TOURO) & $\operatorname{Var}(\mathrm{SEXO}$ *TOURO) & $\operatorname{Var}$ (Error) \\
\hline \multirow[t]{4}{*}{$\begin{array}{l}0 \\
1 \\
2 \\
3 \\
4\end{array}$} & $\begin{array}{l}126,47248511 \\
126,44216031 \\
126,44178085 \\
126,44177733 \\
126,44177733\end{array}$ & $\begin{array}{l}146,70147497 \\
114,60271728 \\
118,45782552 \\
118,04748079 \\
118,04748079\end{array}$ & $\begin{array}{l}49,35127481 \\
84,82641969 \\
80,63757718 \\
80,93492264 \\
80,93492264\end{array}$ & $\begin{array}{l}475,97124397 \\
469,22989295 \\
469,81792240 \\
469,80653064 \\
469,80653064\end{array}$ \\
\hline & \multicolumn{4}{|c|}{ Convergence criteria met, } \\
\hline & \multicolumn{4}{|c|}{ Asymptotic Covariance Matrix of Estimates } \\
\hline & \multicolumn{2}{|c|}{$\operatorname{Var}($ TOURO) } & $\operatorname{Var}(\mathrm{SEXO}$ * TOURO) & $\operatorname{Var}$ (Error) \\
\hline \multicolumn{2}{|c|}{ Var (TOURO) } &, 32898 & $-20264,08059$ & $-52,70243$ \\
\hline \multicolumn{2}{|c|}{$\operatorname{Var}(\mathrm{SEXO}$ *TOURO) } & 08059 & 46003,27191 & $-7558,45243$ \\
\hline \multicolumn{2}{|c|}{ Var (Error) } & 70243 & $-7558,45243$ & 27464,53252 \\
\hline
\end{tabular}


Verifica-se na Tabela 19 que o processo numérico para obter as estimativas REML também convergiu na $5^{3}$ iteração, fornecendo as estimativas dos componentes de variância:

$$
\hat{\sigma}_{\text {touro }}^{2}=118,05 \quad \hat{\sigma}_{\text {sexotrouro }}^{2}=80,93 \quad \hat{\sigma}_{e}^{2}=469,81
$$

Observa-se na Tabela 19 que as estimativas REML dos componentes de variância diferem bastante daquelas obtidas nas Tabelas 17 e 18, estimativas MIVQUE0 e ML respectivamente.

As estimativas REML, conforme visto em 4.2.2.6 são viesadas e diferem pouco das estimativas obtidas na Tabela 16 , que são não viesadas.

Utilizando-se o método REML tem-se novas estimativas, viesadas, dos componentes de variância e nova matriz de covariâncias assintóticas.

As variâncias assintóticas das estimativas dos componentes de variância são dadas conforme a Tabela 19, por:

$$
\operatorname{Var}\left(\hat{\sigma}_{\text {touro }}^{2}\right)=58103,33 \quad \operatorname{Var}\left(\hat{\sigma}_{\text {sexortarro }}^{2}\right)=46003,27 \quad \operatorname{Var}\left(\hat{\sigma}_{e}^{2}\right)=27464,53
$$

Com exceção de $\operatorname{Var}\left(\hat{\sigma}_{e}^{2}\right)$, verifica-se que as variâncias assintóticas das demais estimativas aqui obtidas são muito diferentes daquelas obtidas na Tabela 18.

Apresenta-se na Tabela $19 \mathrm{a}$, as estimativas dos componentes de variância utilizando-se os métodos de estimação disponíveis no PROC VARCOMP.

Tabela 19a. Estimativas dos componentes de variância utilizando o PROC VARCOMP.

\begin{tabular}{lrrc}
\hline Método & $\hat{\sigma}_{\text {touro }}^{2}$ & $\hat{\sigma}_{\text {sexo*touro }}^{2}$ & $\hat{\sigma}_{e}^{2}$ \\
\hline Tipo I & 113,78 & 85,08 & 471,30 \\
MIVQUE0 & 146,14 & 49,16 & 474,15 \\
ML & 78,75 & 7,54 & 471,16 \\
REML & 118,05 & 80,93 & 469,81 \\
\hline
\end{tabular}

Observa-se na Tabela 19a que as estimativas dos componentes de variância obtidas pelos métodos tipo I (método III de Henderson) e REML são próximas, mas as estimativas REML são viesadas enquanto que as estimativas do tipo I são não viesadas. 
O método MIVQUE0 fornece estimativas não viesadas, invariantes e de variância mínima. As estimativas obtidas por esse método diferem de todas as demais estimativas.

As estimativas obtidas pelo método $\mathrm{ML}$, com exceção de $\hat{\sigma}_{e}^{2}$, diferem das demais estimativas obtidas pelo tipo I. As estimativas ML são viesadas e as do tipo I não viesadas. As estimativas ML diferem das obtidas pelo métodos MIVQUE0 e REML.

$\mathrm{O}$ método $\mathrm{ML}$, aqui, subestima as estimativas dos componentes de variância em relação aos outros métodos, com exceção de $\hat{\sigma}_{e}^{2}$.

Os métodos ML e REML são baseados no princípio da máxima verossimilhança. Estes métodos são iterativos e fornecem estimativas diferentes e viesadas.

Não se pode afirmar qual é o melhor método. Uma comparação analítica só é possível para modelos balanceados e ainda assim, deve ser feita para cada modelo linear particular. O método REML tem sido considerado (Searle, 1971; Corbeil \& Searle, 1976; Harville, 1977; Henderson, 1984; Searle et al., 1992; entre outros), o preferido para estimar componentes de variância de dados desbalanceados, conforme já visto em 4.2.2.6.

\subsubsection{Procedimento MIXED}

O PROC MIXED é o procedimento do SAS mais apropriado para a análise de modelos mistos desbalanceados pois este procedimento distingue claramente os efeitos fixos e os aleatórios (SAS, 1992; 1997).

Para o modelo misto $\mathrm{y}=\mathbf{X} \theta+\mathbf{Z u}+e$, descrito em (2), que para o exemplo em questão está caracterizado por $y_{\mathrm{ijk}}=\mu+\alpha_{\mathrm{i}}+\beta_{\mathrm{j}}+\gamma_{\mathrm{ij}}+\varepsilon_{\mathrm{ijk}}$ pode-se escrever:

$$
\mathrm{y}_{\mathrm{ijk}}=\mu+\alpha_{\mathrm{i}}+\varepsilon_{i j k}^{*}
$$

onde $\varepsilon_{i j k}^{*}=\beta_{\mathrm{j}}+\gamma_{\mathrm{ij}}+\varepsilon_{\mathrm{ijk}}$ ou seja, $\varepsilon_{i j k}^{*}$ corresponde aos efeitos aleatórios do modelo. 
Segundo Iemma (1995a), conforme o interesse do pesquisador, o modelo-S pode assumir diferentes caracterizações, desde o modelo mais simples até o modelo completo.

(i) $\quad y=\mathbf{X}_{1} \theta_{1}+\varepsilon^{*}$

$\Leftrightarrow \quad y_{i j k}=\mu+\varepsilon_{i j k}^{*}$

(ii) $\mathrm{y}=\mathbf{X}_{2} \theta_{2}+\varepsilon^{*}$

$\Leftrightarrow$

$\mathrm{y}_{\mathrm{ijk}}=\mu+\alpha_{\mathrm{i}}+\varepsilon_{i j k}^{*}$

Um modelo alternativo equivalente ao modelo-S é aquele denominado modelo-M definido por: $\quad \mathrm{y}_{\mathrm{ijk}}=\mu_{\mathrm{i}}+\varepsilon_{i j \mathrm{k}}^{*}$

onde: $y_{i j k}$ são as variáveis aleatórias para os dados da casela $(i, j)$

$\mu_{\mathrm{i}}$ é a média populacional para a casela (i,j), ou seja, $\mu_{\mathrm{i}}=\mu+\alpha_{\mathrm{i}}$

$\varepsilon_{i j k}^{*}$ são os efeitos aleatórios atribuídos à observação $y_{i j k}$, isto é, $\varepsilon_{i j k}^{*}=\beta_{\mathrm{j}}+\gamma_{\mathrm{ij}}+\varepsilon_{\mathrm{ijk}}$

Considerando os dados da Tabela 3 tem-se esquematicamente, para o modelo-S e para o modelo-M, as Tabelas 20 e 21 respectivamente.

Tabela 20. Esquema para o modelo-S.

\begin{tabular}{lccc}
\hline Fator $A$ & \multicolumn{3}{c}{ Fator $B=$ TOURO } \\
\cline { 2 - 4 } SEXO & $\mathrm{j}=1$ & $\mathrm{j}=2$ & $\mathrm{j}=3$ \\
\hline $\mathrm{i}=1$ & $\mu+\alpha_{1}(5)$ & $\mu+\alpha_{1}(4)$ & $\mu+\alpha_{1}(4)$ \\
$\mathrm{i}=2$ & $\mu+\alpha_{2}(4)$ & $\mu+\alpha_{2}(2)$ & $\mu+\alpha_{3}(3)$ \\
\hline
\end{tabular}

Tabela 21. Esquema para o modelo-M.

\begin{tabular}{cccc}
\hline Fator A & \multicolumn{3}{c}{ Fator B = TOURO } \\
\cline { 2 - 4 } SEXO & $\mathrm{j}=1$ & $\mathrm{j}=2$ & $\mathrm{j}=3$ \\
\hline $\mathrm{i}=1$ & $\mu_{1}(5)$ & $\mu_{1}(4)$ & $\mu_{1}(4)$ \\
$\mathrm{i}=2$ & $\mu_{2}(4)$ & $\mu_{2}(2)$ & $\mu_{2}(3)$ \\
\hline
\end{tabular}

A seguir apresenta-se um programa utilizando o procedimento MIXED, para ajustar os dados da Tabela 3. Como a matriz de covariâncias dos efeitos aleatórios é desconhecida, utiliza-se os métodos de estimação dos componentes de variância disponíveis nesse procedimento, conforme já comentado em 4.3.3. 


\subsubsection{Um programa}

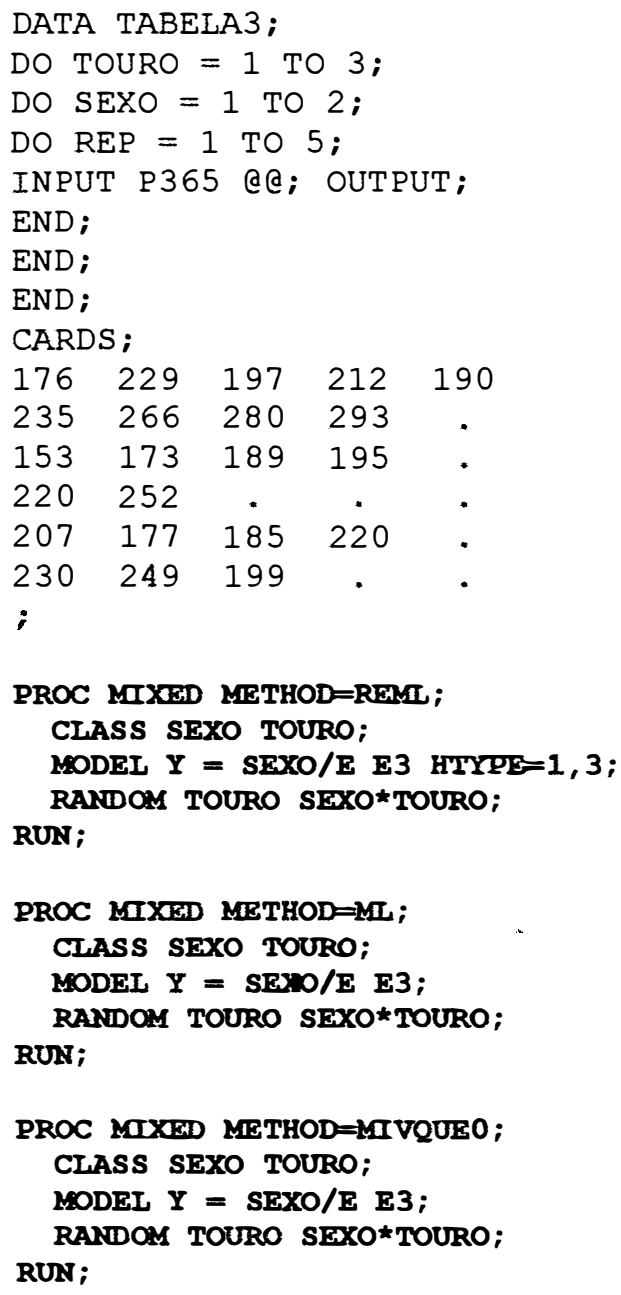

No PROC MIXED os efeitos fixos são especificados no MODEL, e os aleatórios no RANDOM.

O método de estimação default é o REML.

O estrutura de G é definida na opłão RANDOM, e a estrutura default é VC.

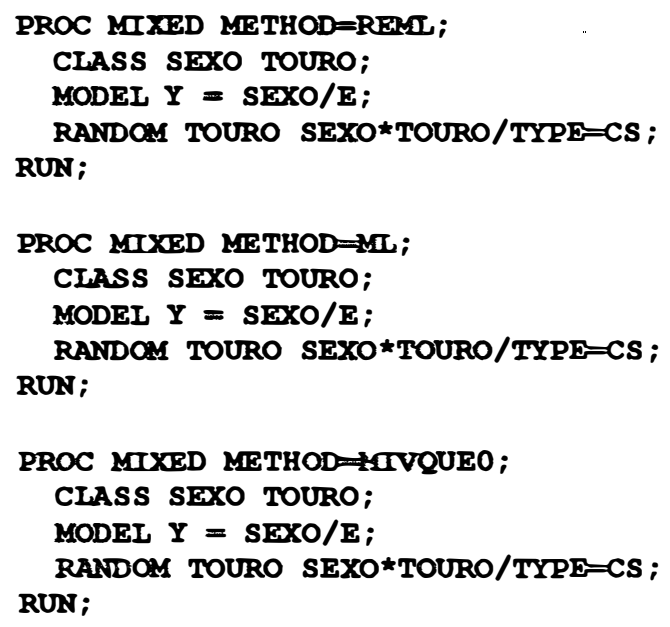

Do lado esquerdo tem-se o programa com a estrutura default, que é a VC, e do lado direito com a escolha da estrutura CS. Procede-se de modo análogo para as demais estruturas de $\mathbf{G}$.

Também foi visto em 4.3.3 que, com PROC MIXED é possível especificar várias estruturas para a matriz de covariâncias dos efeitos aleatórios, matriz G. A estrutura de G default neste procedimento é VC. As outras estruturas de $\mathbf{G}$ consideradas neste trabalho são: CS, TOEP(1), HF. 
No PROC MIXED o método REML é o método de estimação default para se estimar os componentes de variância. Os dois outros métodos disponíveis são: ML e MIVQUE0, conforme descrito em 4.3.3.

Também foi visto em 4.3.3 que, com PROC MIXED é possível especificar várias estruturas para a matriz de covariâncias dos efeitos aleatórios, matriz G. A estrutura de G default neste procedimento é VC. As outras estruturas de $\mathbf{G}$ consideradas neste trabalho são: CS, TOEP(1), HF.

O PROC MIXED fornece mais informações sobre o ajuste do modelo do que o PROC VARCOMP (SAS, 1997), conforme pode ser visto na Tabela 22.

Tabela 22. Informações sobre o ajuste do modelo misto fornecidas pelo PROC MIXED.

\begin{tabular}{|c|c|}
\hline Descrição & Interpretação \\
\hline Observations & número de observações utilizadas na análise. \\
\hline Res Log Likelihood & valor máximo do log da verossimilhança restrita $(\ell)^{(1)}$ \\
\hline Akaike's Information Criterio & $\begin{array}{l}\text { o critério AIC pode ser usado para comparar modelos } \\
\text { com os mesm ss efeitos fixos mas diferentes } \\
\text { estruturas de variância. } O \text { modelo com maior AIC é } \\
\text { considerado o melhor. AIC }=\ell-q \text {, onde } q \text { é o número } \\
\text { de parâmetros de covariâncias existentes em } \mathbf{G} \text { e } R \text {. }\end{array}$ \\
\hline
\end{tabular}

Schwarz's Bayesian Criterion

-2 Res Log Likelihood

Null Model LRT Chi-Square

Null Model LRT DF

Null Model LRT P-Value com o mesmo objetivo do AIC, o modelo com maior BIC é o melhor. BIC $=\ell-\left[q \log \left(n^{*}\right)\right] / 2$, onde $n^{*}=n$ para ML e $n^{*}=n-k$ para REML, $k=r(X)$.

-2 valor máximo do $\log$ da verossimilhança restrita, $-2 \ell$

teste da razão de verossimilhança comparando o modelo em uso com o modelo nulo (modelo ajustado pelo PROC GLM com $\mathbf{R}=\mathbf{I} \sigma^{2}$ ). Esta estatística tem distribuição $\chi^{2}$ com $q-1$ graus de liberdade.

os graus de liberdade para o Null Model LRT Chi-Square que corresponde ao número de parâmetros de covariâncias estimados pelo PROC MIXED menos o número estimado pelo PROC GLM, ou seja q - 1 graus de liberdade.

a probabilidade de observar-se um Null Model LRT ChiSquare. Verifica se o modelo em uso pode ser substituído pelo modelo ordinário. 
Com o PROC MIXED estimam-se os componentes de variância para os efeitos aleatórios do modelo de acordo com o método especificado, bem como as funções estimáveis dos tipos I e III conforme as opções E e E3 especificadas na declaração MODEL e o teste F usando as somas de quadrados dos tipos I e III, para os efeitos fixos.

\subsubsection{Método MIVQUE0}

Através do método MIVQUE, o PROC MIXED fornece as estimativas dos componentes de variância, as funções estimáveis dos tipos I e IIII e os respectivos testes $\mathrm{F}$, conforme descrito em 4.3.3.

\section{a) Para $\mathbf{G}=\mathbf{V C}$}

Para os dados em questão, as funções estimáveis dos tipos I e III são iguais e assim as hipóteses que estão sendo testadas para o fator fixo, sexo, são dadas por:

$$
\mathrm{H}_{0}^{(1-\text { Sexo })}=\mathrm{H}_{0}^{(3-\text { Sexo })}: \alpha_{1}-\alpha_{2}=0
$$

Observa-se que a Tabela 23 fornece as mesmas estimativas dos componentes de variância da Tabela 17, pois ambas utilizam-se da expressão (27) do item 4.2.2.7. Assim, as estimativas obtidas pelo método MIVQUE0, quando $\mathrm{G}=\mathrm{VC}$ são:

$$
\hat{\sigma}_{\text {touro }}^{2}=146,14 \quad \hat{\sigma}_{\text {sexothouro }}^{2}=49,16 \quad \hat{\sigma}_{e}^{2}=474,15
$$

A estrutura $\mathrm{G}=\mathrm{VC}$, default do PROC MIXED é a única possível tanto no PROC GLM através da declaração RANDOM, quanto no PROC VARCOMP.

A matriz de covariâncias de y, dada em (3), é estimada através de

onde

$$
\begin{gathered}
\hat{\mathbf{V}}=\mathbf{Z} \hat{\mathbf{G}} \mathbf{Z}+\mathbf{I}_{9} \hat{\sigma}_{e}^{2} \\
\hat{\mathrm{G}}=\left[\begin{array}{cc}
146,14 \mathbf{I}_{3} & \phi \\
\phi & 49,16 \mathbf{I}_{6}
\end{array}\right]
\end{gathered}
$$


de acordo com as estimativas dos componentes de variância da Tabela 23.

Para o efeito fixo, sexo, neste caso, não está havendo muita diferença entre o nível nominal encontrado nas Tabelas 14 e 23.

Tabela 23. Ajuste do modelo misto através do PROC MIXED utilizando o método MIVQUE0, as opções E e E3 e G=VC.

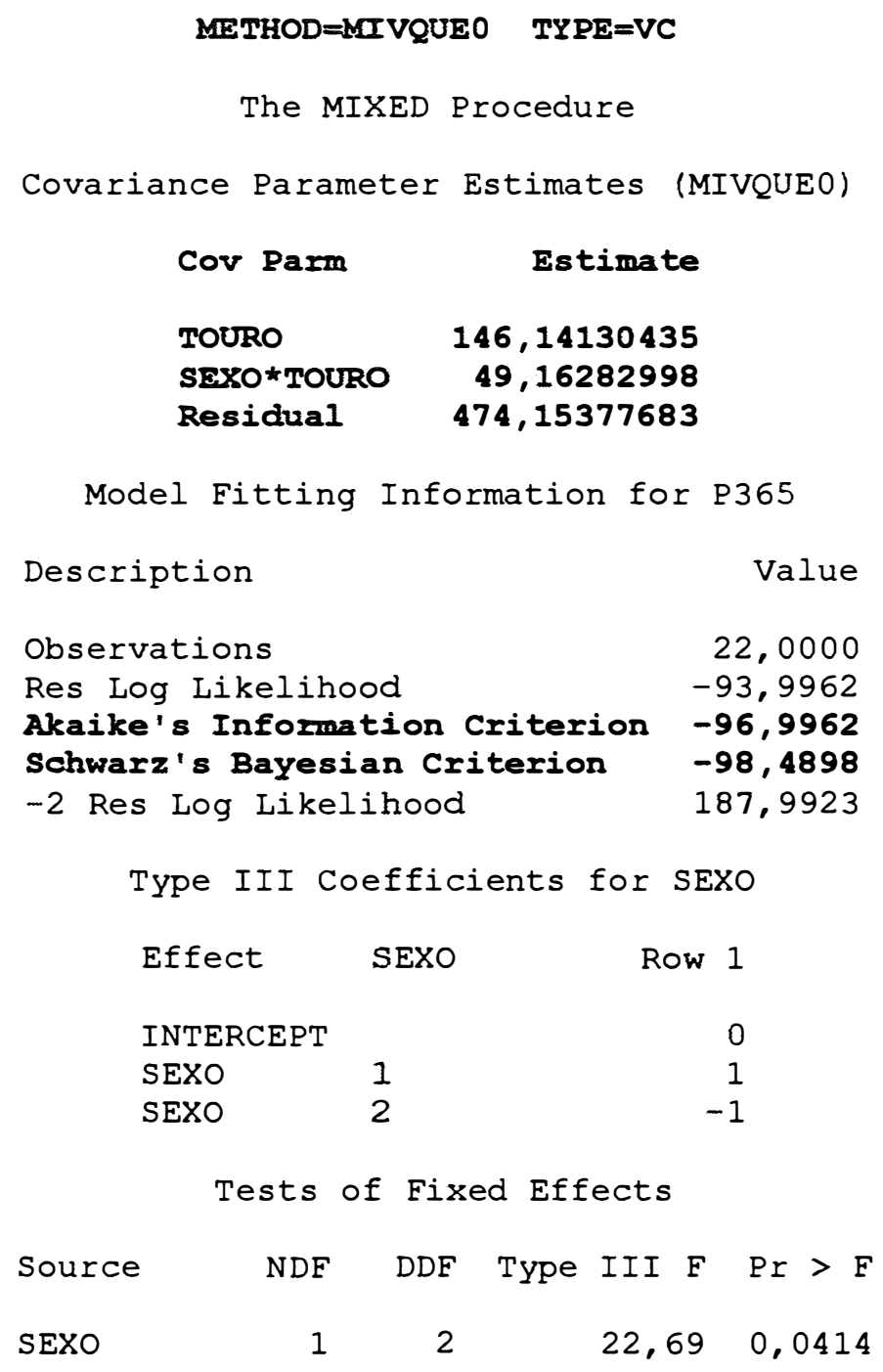

Os critérios AIC e BIC são dados, conforme visto na Tabela 22, por

$$
\begin{aligned}
& \mathrm{AIC}=\ell-\mathrm{q}=-93,9962-3=-96,9962 \\
& \mathrm{BIC}=\ell-[\mathrm{q} \log (\mathrm{n}-\mathrm{k})] / 2=-93,9962-[3 \log (22-2)] / 2=-98,4898
\end{aligned}
$$


Esses critérios podem ser utilizados para comparar modelos como os mesmos efeitos fixos mas diferentes estruturas de covariâncias.

O PROC MIXED não fornece o teste da razão de verossimilhança (Null Model LRT CHI-Square), visto na Tabela 22, para os casos onde $\mathrm{G}=\mathrm{VC}$ (SAS, 1997).

b) $\operatorname{Para} \mathbf{G}=\mathbf{C S}$

Tabela 24. Ajuste do modelo misto através do PROC MIXED utilizando o método MIVQUE0 e $\mathrm{G}=\mathrm{CS}$.

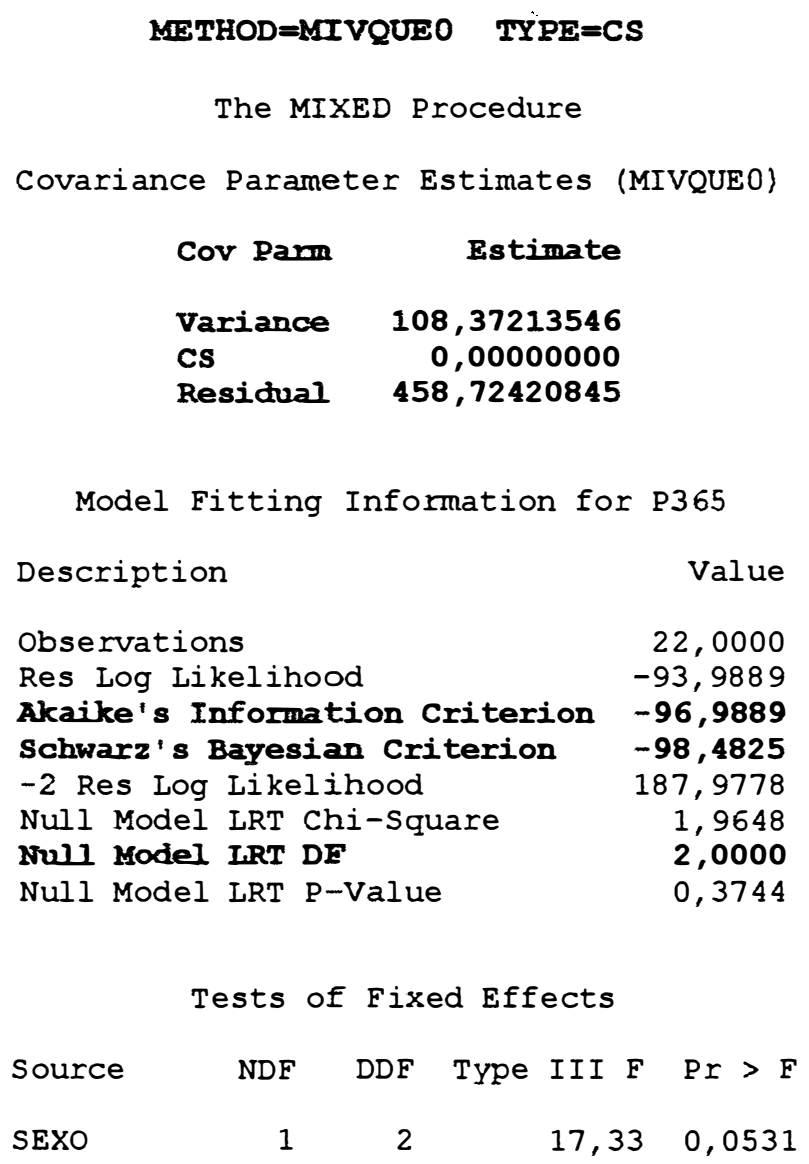

Conforme visto em 4.2.5, para a estrutura $G=C S$, estima-se dois parâmetros de covariância: $\sigma^{2}$ e $\sigma_{1}$. Assim na Tabela 24 tem-se as seguintes estimativas:

$$
\hat{\sigma}^{2}=108,37 \quad ; \quad \hat{\sigma}_{1}=\mathrm{CS}=0 \quad \text { e } \quad \hat{\sigma}_{e}^{2}=458,72
$$


Logo, conforme visto na Tabela 2, tem-se

$$
\hat{\mathrm{G}}=108,37 \mathbf{I}_{9}
$$

A matriz $\hat{\mathrm{G}}$ é assim constituída pois, como se observa na Tabela 24 , a estimativa do parâmetro CS da matriz $\mathbf{G}$, ou seja, $\sigma_{1}$ apresentado na Tabela 2 , é igual a zero.

Nesse caso, os componentes de variância para touro e para interação sexo*touro são iguais a 108,37; pois o valor da estimativa de $\sigma_{1}$ é nula.

Os critérios AIC e BIC são dados, conforme visto na Tabela 22, por

$$
\begin{aligned}
& \mathrm{AIC}=\ell-\mathrm{q}=-93,9889-3=-96,9889 \\
& \mathrm{BIC}=\ell-[\mathrm{q} \log (\mathrm{n}-\mathrm{k})] / 2=-93,9889-[3 \log (22-2)] / 2=-98,4825
\end{aligned}
$$

Os critérios AIC e BIC para as tabelas 23 e 24 são bem próximos e ambas as estruturas estimam três componentes de variância.

Assim, da Tabela 22, $\mathrm{q}-1=3-1=2$ graus de liberdade.

$\mathrm{O}$ teste $\mathrm{F}$ para o efeito fixo, sexo, se altera um pouco, pois conforme comentado em 4.2.3, as estimativas dependem dos métodos e das estruturas de covariâncias adotadas.

Utilizando-se o mesmo método, as estimativas dos componentes mudam dependendo da estrutura escolhida para a matriz de covariâncias.

c) Para $\mathbf{G}=\mathbf{T O E P}(1)$

Conforme visto em 4.2.5, a estrutura $G=\operatorname{TOEP(1)~corresponde~a~diagonal~}$ $\mathrm{G}=\mathbf{I} \sigma^{2}$. Assim na Tabela 25 tem-se as seguintes estimativas:

$$
\hat{\sigma}^{2}=108,37 \text { e } \quad \hat{\sigma}_{e}^{2}=458,72
$$

Logo, conforme visto Tabela 2, tem-se

$$
\hat{\mathrm{G}}=108,37 \mathbf{I}_{9}
$$

Observa-se que essas estimativas são iguais as da Tabela 24, pois na Tabela 24 a estimativa para o parâmetro $\mathrm{CS}, \sigma_{1}$, é zero, logo para o conjunto de dados em questão as opções $G=C S$ e $G=T O E P(1)$ levaram as mesmas estimativas. 
Tabela 25. Ajuste do modelo misto através do PROC MIXED utilizando o método MIVQUE0 e $\mathrm{G}=$ TOEP(1).

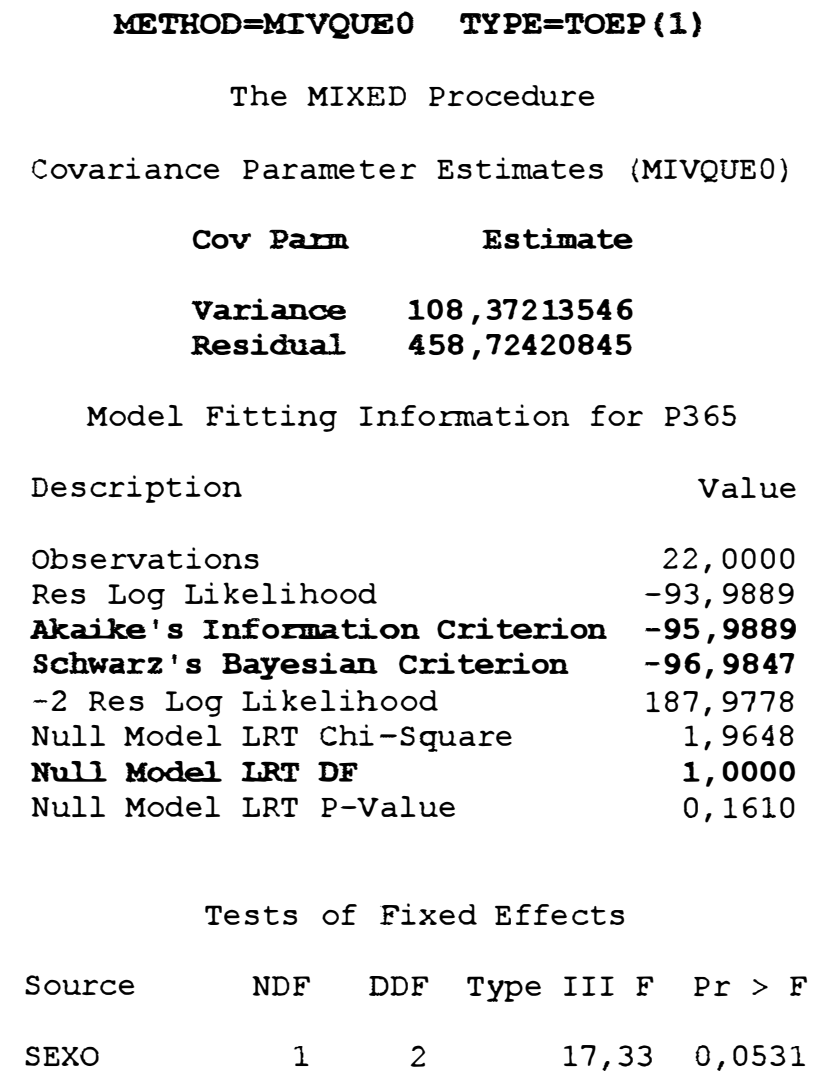

Os critérios AIC e BIC são dados por

$$
\begin{aligned}
& \mathrm{AIC}=\ell-\mathrm{q}=-93,9889-2=-95,9889 \\
& \mathrm{BIC}=\ell-[\mathrm{q} \log (\mathrm{n}-\mathrm{k})] / 2=-93,9889-[2 \log (22-2)] / 2=-96,4847
\end{aligned}
$$

Observa-se nas Tabelas 24 e 25 que, embora suas estimativas de covariâncias tenham sido as mesmas, na Tabela 24 , com o método MIVQUE0 e $\mathrm{G}=\mathrm{CS}$ foram estimados três parâmetros de covariâncias: $\hat{\sigma}^{2}=108,37 ; \hat{\sigma}_{1}=\mathrm{CS}=0$ e $\hat{\sigma}_{e}^{2}=458,72$; enquanto que na Tabela 25 utilizando-se o mesmo método mas com a estrutura $\mathrm{G}=\mathrm{TOEP}(1)$ foram estimados dois componentes: $\hat{\sigma}^{2}=108,37$ e $\hat{\sigma}_{e}^{2}=458,72$. Isso está refletido nos critérios AIC e BIC. 
Utilizando-se os critérios AIC e BIC para as três estruturas VC, CS e TOEP(1), a "melhor", no sentido de maior valor AIC e BIC respectivamente, é a TOEP(1), indicando conforme Tabela 2 que os componentes de variância para touros e para a interação sexo*touro são iguais.

Os testes para o efeito fixo, sexo, nas Tabelas 24 e 25 são iguais pois, eles dependem das estimativas dos componentes de variância, e que, neste caso são iguais.

d) Para G $=$ HF

Para esta estrutura de covariâncias devem ser estimados 9 componentes de variâncias, o valor $\lambda=H F$ e a variância residual, conforme visto em 4.2.5. Tem-se assim 10 graus de liberdade como visto na Tabela 26.

$\mathrm{Na}$ Tabela 26 tem-se as seguintes estimativas:

$$
\begin{aligned}
& \hat{\sigma}_{p}^{2}=6895,72 \text { para } p=1,2, \ldots, 9 \\
& \hat{\lambda}=\mathrm{HF}=3878,84 \\
& \hat{\sigma}_{e}^{2}=458,72
\end{aligned}
$$

Observa-se que as tabelas 24,25 e 26 apresentam as mesmas estimativas $\hat{\sigma}_{e}^{2}$, ou seja a estimativa da variância residual não se alterou quando foram consideradas as estruturas de G iguais a CS, TOEP(1) e HF.

Utilizando-se estas estimativas tem-se que a matriz $\mathrm{G}=\mathrm{HF}$, conforme exemplificado na Tabela 2, é dada por:

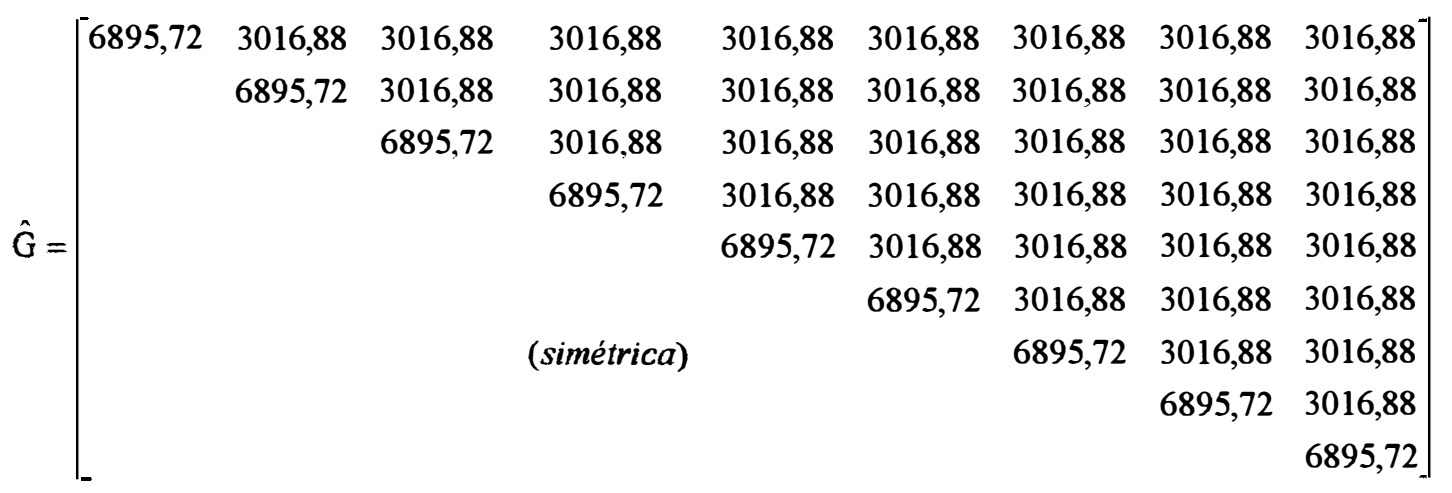


Observa-se, nesse caso, que $\hat{G}$ é do tipo uniforme e pode ser reescrita como:

$$
\hat{\mathrm{G}}=3878,84 \mathrm{I}_{9}+3016,88 \mathrm{~J}_{9}
$$

Assim, as estimativas dadas pela Tabela 26 são:

$$
\hat{\mathrm{G}}=3878,84 \mathbf{I}_{9}+3016,88 \mathbf{J}_{9} \quad \text { e } \quad \hat{\sigma}_{e}^{2}=458,72
$$

Tabela 26. Ajuste do modelo misto através do PROC MIXED utilizando o método MIVQUEO e $\mathrm{G}=\mathrm{HF}$.

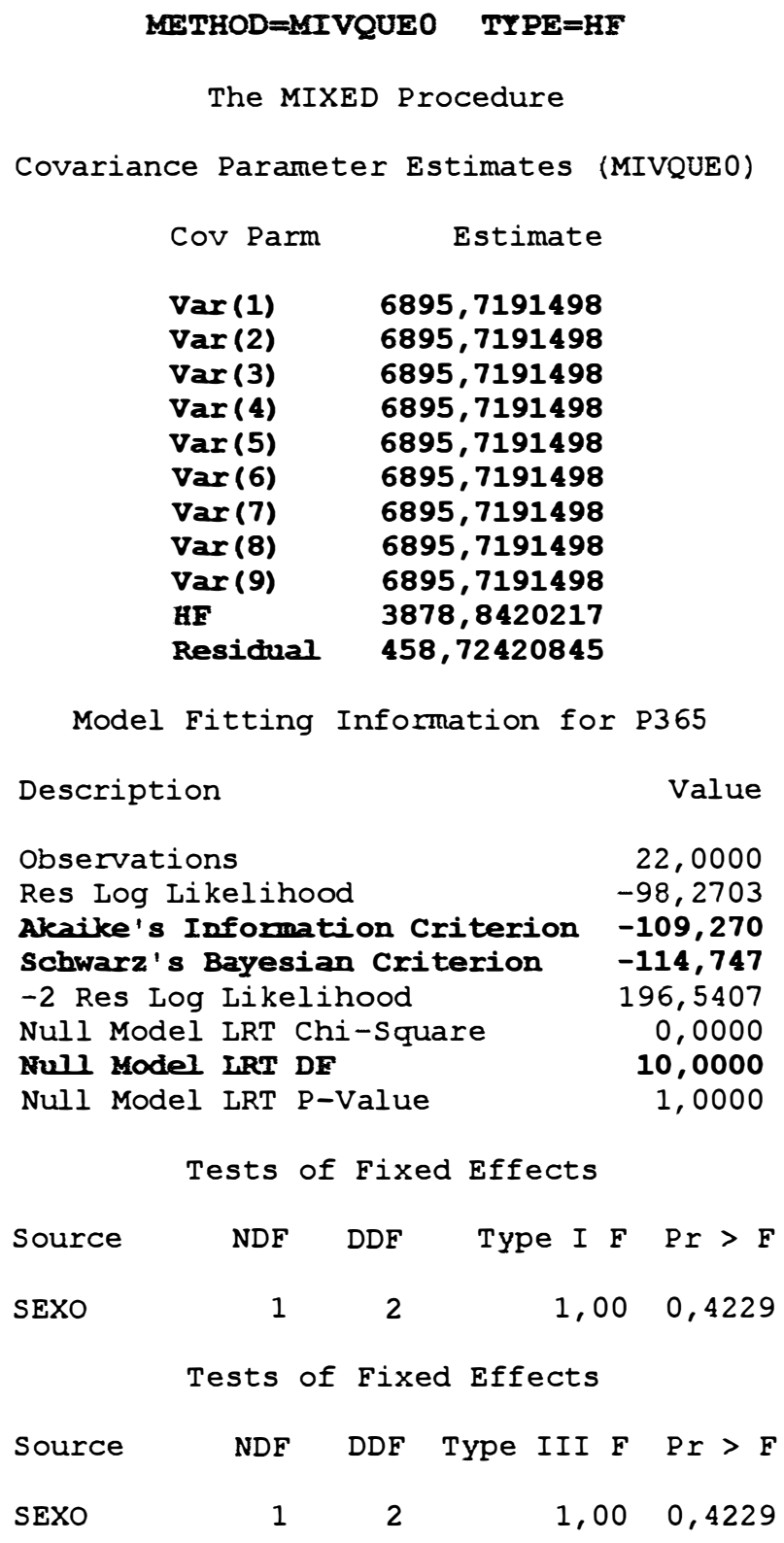


Os valores dos critérios AIC e BIC agora são maiores que os anteriores, ou seja

$$
\begin{aligned}
& \mathrm{AIC}=-98,270-11=-109,270 \\
& \mathrm{BIC}=-98,270-[11 \log (22-2)] / 2=-114,747
\end{aligned}
$$

neste caso, foram estimados 11 elementos, 10 componentes de variância e o $\lambda=H F$.

$\mathrm{O}$ teste para o efeito fixo, sexo, ficou sensivelmente alterado passando a ser não significativo, contrapondo-se aos resultados obtidos nas Tabelas 23,24 e 25 .

$\mathrm{Na}$ Tabela 26a tem-se os resultados dos critérios de informação AIC e BIC, utilizando-se o método MIVQUE0 e as estruturas de G conforme visto em 4.2.5.

Tabela 26a. Resultados do AIC, do BIC e do nível nominal dos testes para o fator de efeitos fixos, sexo, pelo método MIVQUE0 de acordo com a estrutura de G adotada.

\begin{tabular}{ccccc}
\hline Estrutura de G & $\mathrm{q}^{(1)}$ & Valor do AIC & Valor do BIC & $\operatorname{Pr}>$ F (tipo III) \\
\hline VC & 3 & $-96,9962$ & $-98,4898$ & 0,0414 \\
CS & 3 & $-96,9889$ & $-98,4825$ & 0,0531 \\
TOEP(1) & 2 & $-95,9889$ & $-96,9847$ & 0,0531 \\
HF & 11 & $-109,270$ & $-114,747$ & 0,4229
\end{tabular}

(1) número de parâmetros de covariâncias estimados no modelo.

Observando-se a Tabela 26a, a estrutura com o menor valor de AIC e BIC, considerada a "pior", é a estrutura de $\mathrm{G}=\mathrm{HF}$. Conforme visto, com esta estrutura tem-se a maior alteração no nível nominal do teste para o efeito fixo, sexo.

Verifica-se pelos valores AIC e BIC na Tabela 26a que apenas a estrutura HF não é indicada para os dados. Dentre as mais indicadas há um ligeiro favoritismo para TOEP(1).

Não se pode perder de vista, no entanto que outras estruturas, talvez importantes, não foram aqui consideradas. Tudo depende da habilidade do pesquisador na busca da estrutura mais adequada para a estrutura dos dados. 


\subsubsection{Método ML}

As estimativas ML são os valores que maximizam a função de verossimilhança dos dados ou que, equivalentemente minimizam a função objetivo que é $-2 l$ de acordo com a expressão (20) do item 4.2.2.5.

As estimativas ML são estabelecidas utilizando-se o algoritmo de NewtonRaphson, que usa a primeira e segunda derivadas da função objetivo para iterativamente estabelecer seu mínimo.

O PROC MIXED fornece as estimativas ML, as funções estimáveis dos tipos I e III e os testes F utilizando-se as somas de quadrados dos tipos I e III para os efeitos fixos.

Os resultados obtidos através do PROC MIXED utilizando o método ML e as estruturas de $\mathbf{G}$ consideradas, conforme já dito em 4.2 .5 , são dadas a seguir.

a) $\operatorname{Para} \mathbf{G}=\mathbf{V C}$

Para o conjunto modelo-dados, em questão, as funções estimáveis dos tipos I e III para o fator de efeitos fixos, sexo, são iguais e assim as hipóteses que estão sendo testadas, são dadas por:

$$
\mathrm{H}_{0}^{(1-\text { Sexo })}=\mathrm{H}_{0}^{(3-\text { Sexo })}: \alpha_{1}-\alpha_{2}=0
$$

Observa-se na Tabela 27 que o processo numérico para obter as estimativas ML convergiu na segunda iteração fornecendo, praticamente, as mesmas estimativas dos componentes de variância da Tabela 18, pois ambos os procedimentos, VARCOMP e MIXED, utilizam-se da expressão (22) do item 4.2.2.5, para obter as estimativas ML. Assim as estimativas pelo método $\mathrm{ML}$, quando $\mathbf{G}=\mathbf{V C}$ são:

$$
\hat{\sigma}_{\text {touro }}^{2}=78,74 \quad \hat{\sigma}_{\text {sexo touro }}^{2}=7,54 \quad \hat{\sigma}_{e}^{2}=471,18
$$

Comparando-se as estimativas da Tabela 27 com as da Tabela 23, observa-se que as estimativas ML são menores que as estimativas MIVQUE0, no entanto, conforme 
comentado em 4.2.2.5 e 4.2.2.8 respectivamente, as estimativas MIVQUE0 são não viesadas enquanto que as ML viesadas.

Tabela 27. Ajuste do modelo misto através do PROC MIXED utilizando o método ML, as opções E e E3 e $\mathrm{G}=\mathrm{VC}$.

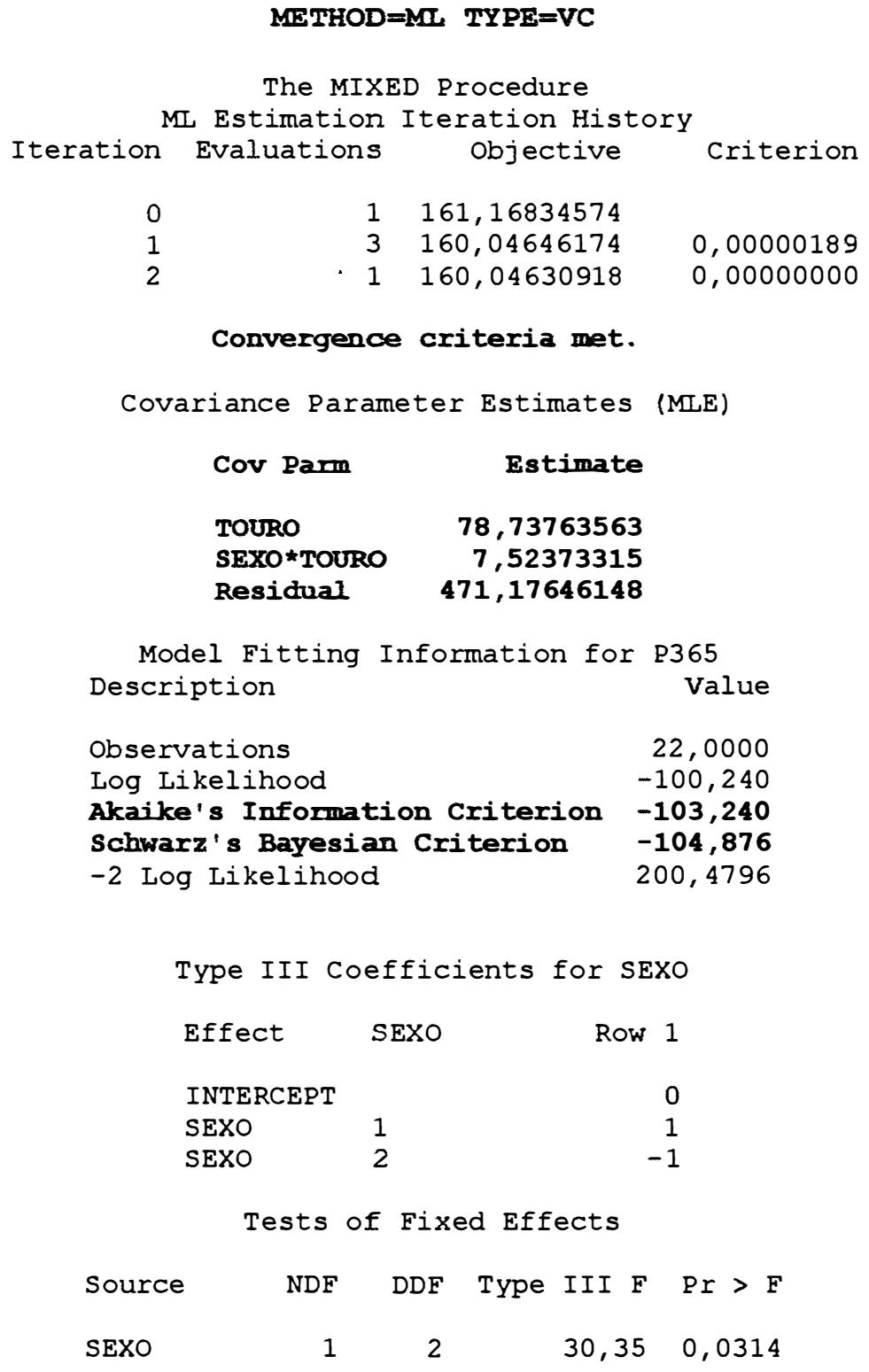

A matriz de covariâncias de y, dada em (3), é estimada por $\hat{\mathbf{V}}=\mathbf{Z} \hat{\mathrm{G}} \mathbf{Z}{ }^{\prime}+\hat{\sigma}_{e}^{2} \mathbf{I}_{9}$ onde, como visto na Tabela 2 e utilizando-se as estimativas da Tabela 27, tem-se 


$$
\hat{\mathrm{G}}=\left[\begin{array}{cc}
78,74 \mathbf{I}_{3} & \phi \\
\phi & 7,54 \mathbf{I}_{6}
\end{array}\right]
$$

Observa-se na Tabela 27, que foram estimados três componentes de variância. Assim, os critérios AIC e BIC são dados, conforme visto na Tabela 22, por

$$
\begin{aligned}
& \mathrm{AIC}=\ell-\mathrm{q}=-100,240-3=-103,240 \\
& \mathrm{BIC}=\ell-(\mathrm{q} \log \mathrm{n}) / 2=-100,240-(3 \log 22) / 2=-104,876
\end{aligned}
$$

Esses critérios podem ser utilizados para comparar modelos com os mesmos efeitos fixos mas com diferentes estruturas de covariâncias.

Comparando-se os valores do AIC e BIC, das Tabelas 23 e 27, observa-se que os valores do método ML são menores, no entanto não se pode perder de vista que esses critérios devem ser utilizados para comparar modelos com mesmos efeitos fixos e diferentes estruturas de covariâncias e não métodos de estimação. Métodos de estimação podem ser comparados através da adequação de suas metodologias, ao conjunto de dados em estudo.

O nível nominal do teste para o fator de efeitos fixos, sexo, altera-se de 0,0414 usando o MIVQUE0, para 0,0314 pelo ML.

b) $\operatorname{Para} \mathbf{G}=\mathbf{C S}$

Observa-se na Tabela 28 que o processo numérico para obter as estimativas de máxima verossimilhança não convergiu.

Comparando-se as Tabelas 24 e 28 , para $\mathbf{G}=\mathbf{C S}$, verifica-se que as estimativas MIVQUE0 da Tabela 24 devem ser as preferidas em relação as estimativas ML, pois o método ML não convergiu. 
Tabela 28. Ajuste do modelo misto através do PROC MIXED utilizando o método ML e $\mathrm{G}=\mathrm{CS}$.

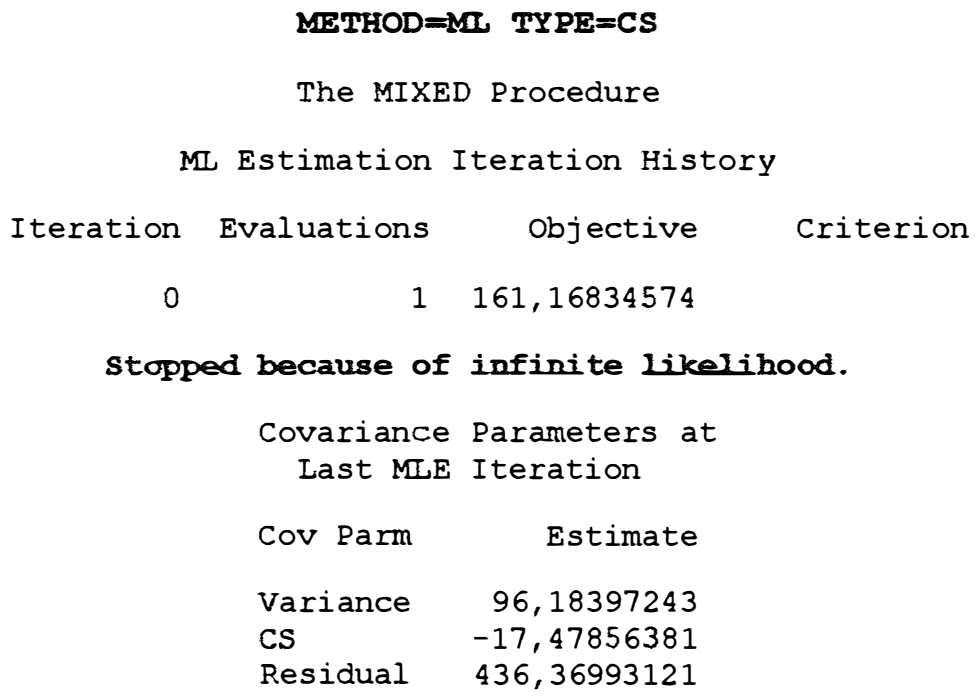

c) $\operatorname{Para} \mathbf{G}=\operatorname{TOEP}(1)$

Observa-se na Tabela 29, que o processo numérico para obter as estimativas ML convergiu na segunda iteração.

Conforme visto em 4.2.5, a estrutura $G=\operatorname{TOEP}(1)$ corresponde a diagonal $\mathrm{G}=\mathbf{I} \sigma^{2}$. Assim na Tabela 29 tem-se as seguintes estimativas:

$$
\hat{\sigma}^{2}=47,60 \text { e } \quad \hat{\sigma}_{e}^{2}=461,11
$$

Logo, conforme visto na Tabela 2, tem-se

$$
\hat{\mathrm{G}}=47,60 \mathrm{I}_{9}
$$

Comparando-se essas estimativas com as da Tabela 25 , observa-se uma grande diferença entre elas.

Os critérios AIC e BIC são dados, conforme visto na Tabela 22, por

$$
\begin{aligned}
& \mathrm{AIC}=\ell-\mathrm{q}=-100,290-2=-102,290 \\
& \mathrm{BIC}=\ell-(\mathrm{q} \log \mathrm{n}) / 2=-100,290-(2 \log 22) / 2=-103,381
\end{aligned}
$$


As estruturas de G, VC e TOEP(1), podem ser comparadas utilizando-se os valores de AIC e BIC. Observa-se que na Tabela 27, foram estimados 3 componentes de variância enquanto que na Tabela 29, foram estimados 2 componentes. Isso está refletido nos valores de AIC e BIC.

Verifica-se também que o nível nominal dos testes para o efeito fixo, sexo, também pouco se alterou.

Tabela 29. Ajuste do modelo misto através do PROC MIXED utilizando o método ML e $\mathrm{G}=\mathrm{TOEP}(1)$.

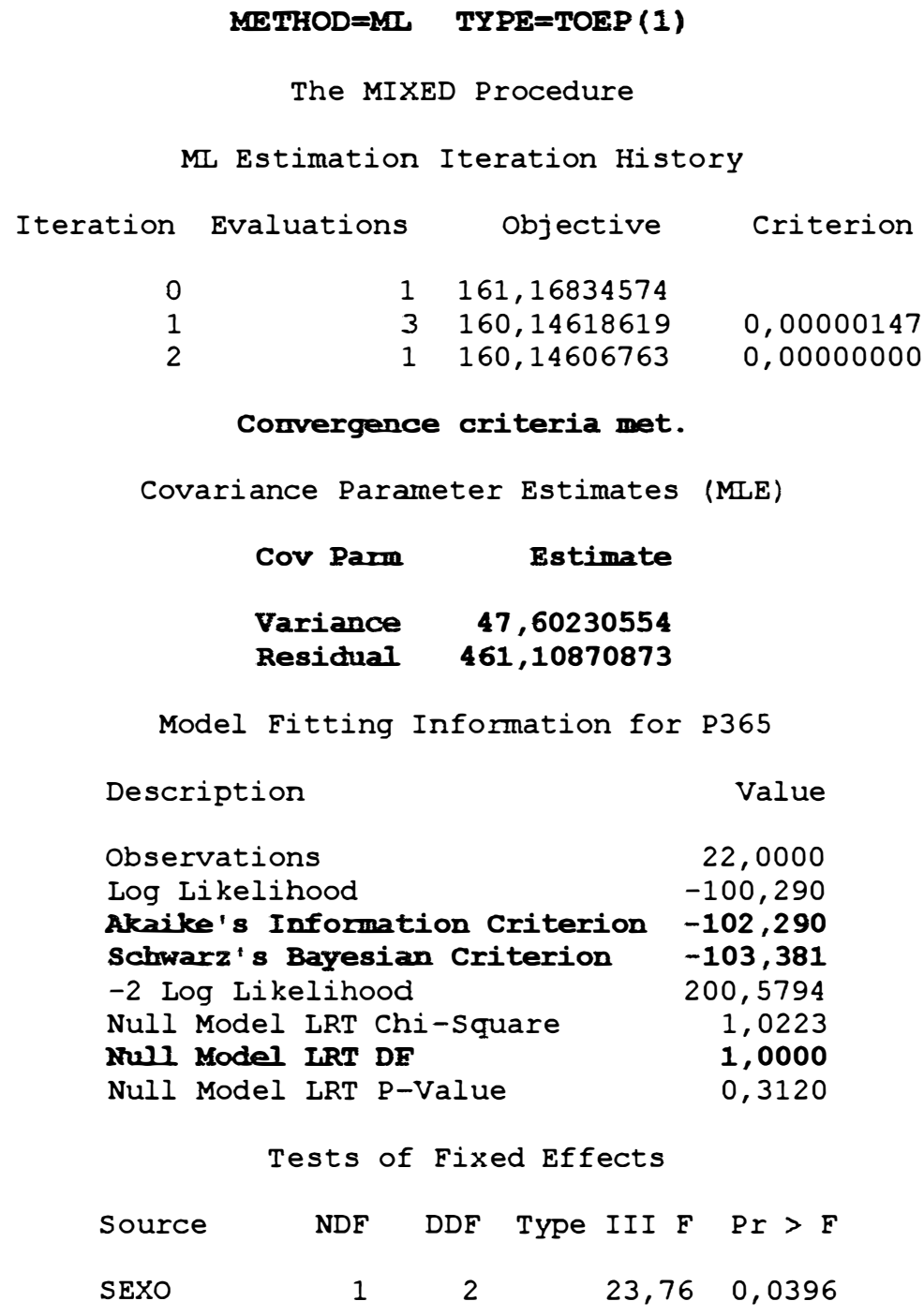




\section{d) $\operatorname{Para} \mathbf{G}=\mathbf{H F}$}

Observa-se na Tabela 30, que o procedimento númerico para obter as estimativas ML para $G=H F$, não convergiu.

Comparando-se as Tabelas 26 e 30 , para $\mathrm{G}=\mathrm{HF}$, verifica-se que as estimativas MIVQUE0 da Tabela 26 devem ser preferidas em relação as estimativas ML, pois o método ML não convergiu.

Tabela 30. Ajuste do modelo misto através do PROC MIXED utilizando o método ML e $\mathrm{G}=\mathrm{HF}$.

\section{METHOD $=M I$ TYPE $=\mathrm{HF}$}

The MIXED Procedure

ML Estimation Iteration History

$\begin{array}{crrr}\text { Iteration } & \text { Evaluations } & \text { Objective } & \text { Criterion } \\ 0 & 1 & 161,16834574 & \\ 1 & 4 & 167,02483402 & \\ 2 & 1 & 165,86328590 & 1,96268949 \\ 3 & 1 & 165,27829296 & 0,28545460 \\ 4 & 1 & 165,05471826 & 0,02824402 \\ \ldots & & & \\ 33 & 0 & 139,64756835 & 5,8558482 \mathrm{E} 21 \\ 34 & 0 & 139,64756835 & 5,8558482 \mathrm{E} 21 \\ 35 & 0 & 139,64756835 & 5,8558482 \mathrm{E} 21 \\ 36 & 0 & 139,64756835 & 5,8558482 \mathrm{E} 21 \\ 37 & 0 & 139,64756835 & 5,8558482 \mathrm{E} 21\end{array}$

Did not converge.

$\begin{array}{lr}\begin{array}{c}\text { Covariance } \\ \text { Last MLE }\end{array} & \begin{array}{r}\text { Parameters at } \\ \text { Iteration }\end{array} \\ \text { Cov Parm } & \text { Estimate } \\ & \\ \text { Var(1) } & 1385,1934331 \\ \text { Var(2) } & 1988,1538617 \\ \text { Var(3) } & 1859,6219426 \\ \text { Var(4) } & 1435,5045240 \\ \text { Var(5) } & 2084,6025642 \\ \text { Var(6) } & 1992,0880095 \\ \text { Var(7) } & 1578,1643353 \\ \text { Var(8) } & 2077,8350157 \\ \text { Var(9) } & 2026,8852099 \\ \text { HF } & 35,97796572 \\ \text { Residual } & 476,43844790\end{array}$


$\mathrm{Na}$ Tabela 30a tem-se os resultados dos critérios de informação AIC e BIC, utilizando-se o método ML e as estruturas de G apresentadas em 4.2.5.

Tabela 30a. Resultados do AIC, do BIC e do nível nominal dos testes para o fator de efeitos fixos, sexo, pelo método ML de acordo com a estrutura de G adotada.

\begin{tabular}{ccccc}
\hline Estrutura de G & $\mathrm{q}^{(1)}$ & Valor do AIC & Valor do BIC & $\operatorname{Pr}>$ F (tipo III) \\
\hline VC & 3 & $-103,240$ & $-104,876$ & 0,0314 \\
CS & $(*)$ & $(*)$ & $(*)$ & $(*)$ \\
TOEP(1) & 2 & $-102,290$ & $-103,381$ & 0,0396 \\
HF & $(*)$ & $(*)$ & $(*)$ & $(*)$ \\
\hline
\end{tabular}

(1) número de parâmetros de covariâncias estimados no modelo.

(*) processo numérico para obter as estimativas ML não convergiu

Observando-se a Tabela 30a, a estrutura com o maior valor do AIC e do BIC, considerada a "melhor", é a estrutura de G = TOEP(1), mas os valores do AIC e BIC para $\mathrm{G}=\mathrm{VC}$ são próximos, assim como também são sróximos os níveis dos testes. Logo, a escolha da estrutura de $\mathrm{G}$ mais apropriada para os dados da Tabela 3, não deve ser exclusivamente baseada nestes critérios, devem-se também e principalmente considerar a natureza dos dados e a experiência do pesquisador (Wolfinger, 1993).

\subsubsection{Método REML}

As estimativas REML são os valores que maximizam a função de verossimilhança restrita dos dados ou que, equivalentemente, minimizam a função objetivo que é -2 vezes o logaritmo dessa verossimilhança, expressão (25) do item 4.2.2.6. 
As estimativas REML são estabelecidas utilizando-se o algoritmo de NewtonRaphson, que usa a primeira e segunda derivadas da função objetivo, para, iterativamente, estabelecer seu mínimo.

O PROC MIXED fornece as estimativas REML como default, para os componentes de variância. Fornece também as funções estimáveis dos tipos I e III e os testes F utilizando-se as somas de quadrados dos tipos I e III para o fator de efeitos fixos.

Os resultados obtidos através do PROC MIXED, utilizando-se o método REML e as estruturas de $\mathbf{G}$ consideradas conforme visto em 4.2.5, são apresentados nas Tabelas 31 a 34.

a) $\operatorname{Para} \mathbf{G}=\mathbf{V C}$

Observa-se, na Tabela 31, que o processo numérico para obtenção das estimativas REML convergiu na terceira iteração, fornecendo as seguintes estimativas:

$$
\dot{o}_{\text {touro }}^{2}=118,05 \quad \hat{\sigma}_{\text {sexo }}^{2}=80,93 \quad \hat{\sigma}_{e}^{2}=469,81
$$

Comparando-se as Tabelas 31 e 19, observa-se que elas apresentam as mesmas estimativas dos componentes de variância, pois ambos os procedimentos, VARCOMP e MIXED, utilizam-se da expressão (25) do item 4.2.2.6, para obter as estimativas REML.

A estimativa de $G$, conforme visto na Tabela 2 e utilizando-se as estimativas dos componentes de variância da Tabela 31, é dada por:

$$
\hat{\mathbf{G}}=\left[\begin{array}{cc}
118,05 \mathbf{I}_{3} & \phi \\
\phi & 80,93 \mathbf{I}_{6}
\end{array}\right]
$$

Como pode ser observado na Tabela 31 , as funções estimáveis dos tipos I e III são iguais e assim as hipóteses que estão sendo testadas para o fator fixo, sexo, são dadas por:

$$
\mathrm{H}_{0}^{(1-\operatorname{Sexo})}=\mathrm{H}_{0}^{(3-\operatorname{Sexo})}: \alpha_{1}-\alpha_{2}=0
$$


Tabela 31. Ajuste do modelo misto através do PROC MIXED utilizando o método REML, as opções E e E3 e $\mathrm{G}=\mathrm{VC}$.

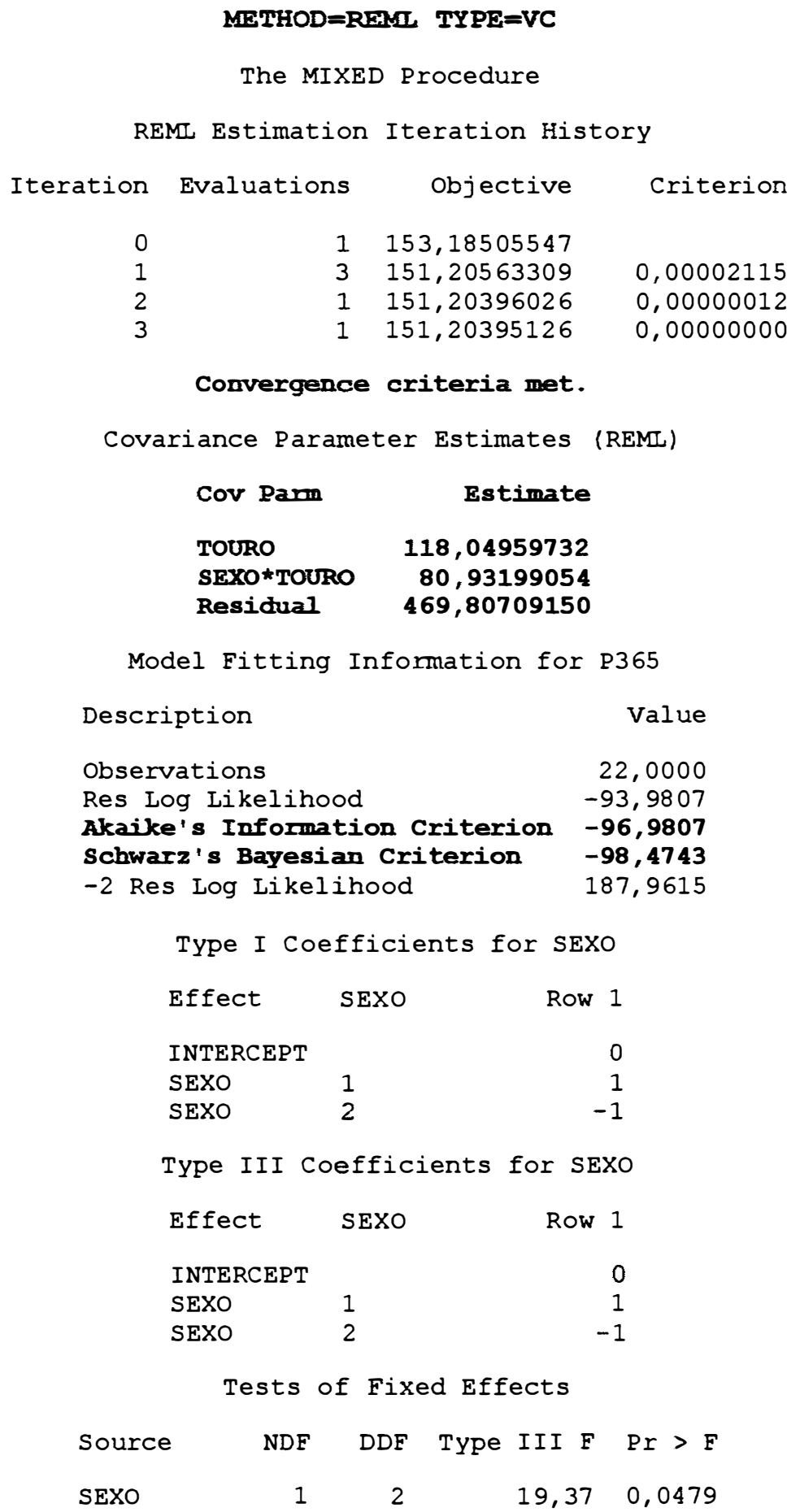


Observa-se na Tabela 31, que foram estimados três componentes de variância. Assim, os critérios AIC e BIC são dados, conforme visto na Tabela 22, por

$$
\begin{aligned}
& \mathrm{AIC}=\ell-\mathrm{q}=-93,9807-3=-96,9807 \\
& \mathrm{BIC}=\ell-[\mathrm{q} \log (\mathrm{n}-\mathrm{k})] / 2=-93,9807-[3 \log (22-2)] / 2=-98,4743
\end{aligned}
$$

que podem ser utilizados para comparar modelos como os mesmos efeitos fixos mas diferentes estruturas de covariâncias.

Verifica-se que as estimativas MIVQUE0, ML e REML, apresentadas respectivamente nas Tabelas 23, 27 e 31, são diferentes.

b) $\operatorname{Para} \mathbf{G}=\mathbf{C S}$

Verifica-se na Tabela 32, que o processo numérico para obtenção das estimativas REML convergiu na segunda iteração.

Conforme visto em 4.2 .5 , para a estrutura $G=C S$, estimam-se dois parâmetros de covariâncias: $\sigma^{2}$ e $\sigma_{1}$. Assim, na Tabela 32, tem-se as seguintes estimativas:

$$
\hat{\sigma}^{2}=99,28 \quad ; \quad \hat{\sigma}_{1}=\mathrm{CS}=0 \quad \text { e } \quad \hat{\sigma}_{e}^{2}=468,05
$$

Logo, conforme visto na Tabela 2, tem-se

$$
\hat{G}=99,28 \mathbf{I}_{9}
$$

A matriz $\hat{G}$ é assim constituída pois, como se observa na Tabela 32, a estimativa do parâmetro CS da matriz $\mathbf{G}$, ou seja, $\sigma_{1}$ apresentado na Tabela 2 , é igual a zero.

Nesse caso, os componentes de variância para touros e para interação sexo*touro são iguais a 99,28; pois o valor da estimativa de $\sigma_{1}$ é nula.

Os critérios AIC e BIC são dados, conforme visto na Tabela 22, por

$$
\begin{aligned}
& \mathrm{AIC}=\ell-\mathrm{q}=-93,9852-3=-96,9852 \\
& \mathrm{BIC}=\ell-[\mathrm{q} \log (\mathrm{n}-\mathrm{k})] / 2=-93,9852-[3 \log (22-2)] / 2=-98,4788
\end{aligned}
$$

pois, da Tabela 32, $\mathrm{q}-1=3-1=2$ graus de liberdade.

Observa-se nas Tabelas 24 e 32, que as estimativas MIVQUEO e REML, respectivamente, são diferentes e o nível nominal dos testes para o efeito fixo, sexo, praticamente não se altera. 
As estimativas REML da Tabela 32 não podem ser comparadas com as estimativas ML da Tabela 28, pois na Tabela 28 o processo numérico para obter as estimativas ML não convergiu.

Tabela 32. Ajuste do modelo misto através do PROC MIXED utilizando o método REML e $\mathrm{G}=\mathrm{CS}$.

\section{METHOD $=$ REMI TYPE $=C S$}

The MIXED Procedure

REML Estimation Iteration History

$\begin{array}{rrrr}\text { Iteration } & \text { Evaluations } & \text { Objective } & \text { Criterion } \\ 0 & 1 & 153,18505547 & \\ 1 & 3 & 151,21295446 & 0,00000264 \\ 2 & 1 & 151,21294653 & 0,00000000\end{array}$

Convergence criteria met.

Covariance Parameter Estimates (REMC)

Cov Parm Estimate

Variance $\quad 99,28285907$

CS $\quad-0,00000000$

Residual 468,04519928

Model Fitting Information for P365

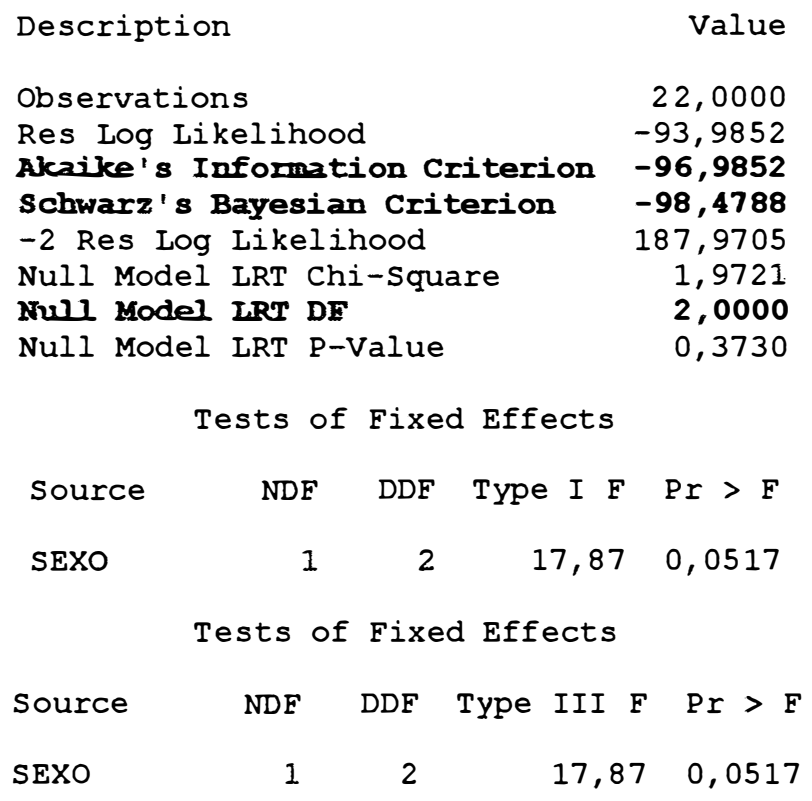


c) $\operatorname{Para} \mathbf{G}=\operatorname{TOEP}(1)$

Tabela 33. Ajuste do modelo misto através do PROC MIXED utilizando o método REML e $G=$ TOEP(1).

\section{METHOD=REMI TYPE=TOEP (1) \\ The MIXED Procedure}

REML Estimation Iteration History

$\begin{array}{rrrr}\text { Iteration } & \text { Evaluations } & \text { Objective } & \text { Criterion } \\ & 1 & 153,18505547 & \\ 1 & 3 & 151,21309205 & 0,00000190 \\ 2 & 1 & 151,21294658 & 0,00000000 \\ & & & \\ & \text { Convergence criteria met. }\end{array}$

Covariance Parameter Estimates (REMU)

Cov Parm Estimate

Variance $\quad 99,26160087$

Residual 468,05775236

Model Fitting Information for P365

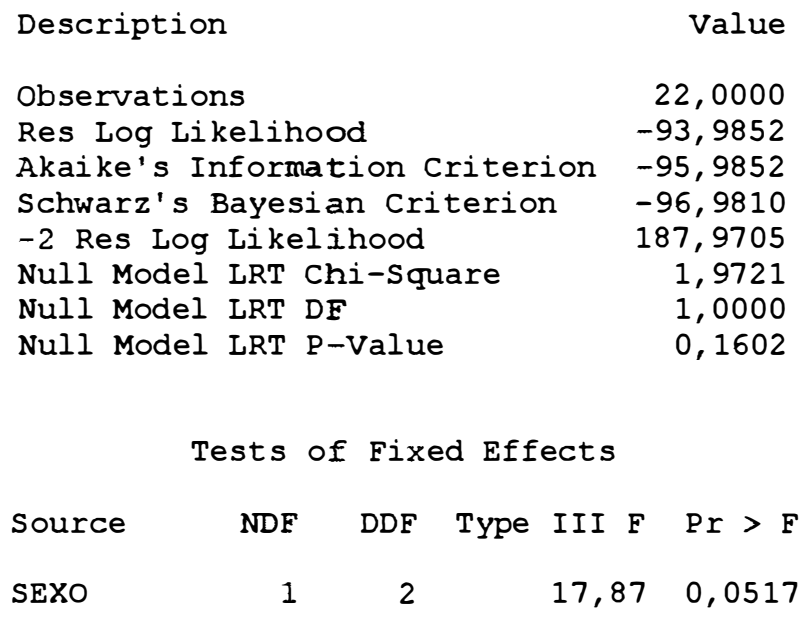

Observa-se na Tabela 33 que o processo numérico para encontrar as estimativas também convergiu na segunda iteração. 
Conforme visto em 4.2.5, a estrutura $G=\operatorname{TOEP}(1)$ corresponde a diagonal $\mathrm{G}=\mathbf{I} \sigma^{2}$. Assim na Tabela 33 tem-se as seguintes estimativas:

$$
\hat{\sigma}^{2}=99,26 \text { e } \quad \hat{\sigma}_{e}^{2}=468,06
$$

Logo, conforme visto Tabela 2, tem-se

$$
\hat{\mathrm{G}}=99,26 \mathbf{I}_{9}
$$

Como pode ser visto essas estimativas são iguais as da Tabela 32, pois na Tabela 32 a estimativa para o parâmetro $\mathrm{CS}, \sigma_{1}$, é zero, logo para o conjunto de dados em questão as opções $G=C S$ e $G=\operatorname{TOEP}(1)$ levaram as mesmas estimativas.

Os critérios AIC e BIC são dados, conforme visto na Tabela 22, por

$$
\begin{aligned}
& \mathrm{AIC}=\ell-\mathrm{q}=-93,9852-2=-95,9852 \\
& \mathrm{BIC}=\ell-[\mathrm{q} \log (\mathrm{n}-\mathrm{k})] / 2=-93,9852-[2 \log (22-2)] / 2=-96,9810
\end{aligned}
$$

Verifica-se nas Tabelas 32 e 33 que, embora suas estimativas de variâncias tenham sido as mesmas, na Tabela 32 com método REML e $G=C S$ foram estimados três parâmetros de covariância: $\hat{\sigma}^{2}=99,28 ; \hat{\sigma}_{1}=\mathrm{CS}=0$ e $\hat{\sigma}_{e}^{2}=468,05$; enquanto que na Tabela 33 utilizando-se o mesmo método mas com a estrutura $G=T$ TCEP(1) foram estimados dois componentes: $\hat{\sigma}^{2}=99,26$ e $\hat{\sigma}_{e}^{2}=468,06$. Isso está refletido nos critérios AIC e BIC.

Utilizando-se os critérios AIC e BIC para comparar as três estruturas VC, CS e TOEP(1), com o método REML, observa-se que "a melhor", no sentido de maior valor AIC e BIC respectivamente, é a TOEP(1), indicando, conforme Tabela 2, que os componentes de variância para touros e para a interação sexo*touro são iguais.

Os testes para o efeito fixo, sexo, nas Tabelas 32 e 33 são iguais pois, eles dependem das estimativas dos componentes de variância, e que, neste caso são iguais.

d) $\operatorname{Para} \mathbf{G}=\mathbf{H F}$

Para a estrutura de covariâncias $G=H F$ devem ser estimados 9 componentes de variâncias, o valor $\lambda=$ HF e a variância residual, conforme visto em 4.2.5. Tem-se assim 10 graus de liberdade como pode ser visto na Tabela 34. 
Tabela 34. Ajuste do modelo misto através do PROC MIXED utilizando o método REML e $G=H F$.

\section{METHOD=RMMT TYPE=HF}

The MIXED Procedure

REMI Estimation Iteration History

$\begin{array}{rrrr}\text { Iteration } & \text { Evaluations } & \text { objective } & \text { Criterion } \\ 0 & 1 & 153,18505547 & \\ 1 & 4 & 153,18505547 & \\ 2 & 1 & 152,08302969 & 1,87321951 \\ 3 & 1 & 151,49996043 & 0,28203925 \\ 4 & 1 & 151,26910591 & 0,03022674 \\ 5 & 1 & 151,21686701 & 0,00147636 \\ 6 & 1 & 151,21297698 & 0,00001019 \\ 7 & 1 & 151,21294653 & 0,00000000\end{array}$

Convergence criteria met.

Covariance Parameter Estimates
$\begin{array}{lr}\text { Cov Pamm } & \text { Estimate } \\ & \\ \text { Var (1) } & 7035,8419248 \\ \text { Var (2) } & 7035,8419256 \\ \text { Var (3) } & 7035,8419251 \\ \text { Var (4) } & 7035,8419254 \\ \text { Var (5) } & 7035,8419257 \\ \text { Var (6) } & 7035,8419255 \\ \text { Var (7) } & 7035,8419249 \\ \text { Var (8) } & 7035,8419253 \\ \text { Var (9) } & 7035,8419253 \\ \text { GF } & 99,28217763 \\ \text { Residual } & 468,04560162\end{array}$

Model Fitting Information for P365

$\begin{array}{lr}\text { Description } & \text { Value } \\ \text { Observations } & 22,0000 \\ \text { Res Log Likelihood } & -93,9852 \\ \text { Akaike's Information Criterion } & -104,985 \\ \text { Schwarz's Bayesian Criterion } & -110,462 \\ \text {-2 Res Log Likelihood } & 187,9705 \\ \text { Null Model LRT Chi-Square } & 1,9721 \\ \text { Null Model IRT DE } & 10,0000 \\ \text { Null Model LRT P-Value } & 0,9965\end{array}$

Tests of Fixed Effects

Source NDF DDF Type I F Pr $>$ F

$\begin{array}{lllll}\text { SEXO } & 1 & 2 & 17,87 & 0,0517\end{array}$


Com relação a Tabela 34, verifica-se que o processo numérico para obter as estimativas convergiu na sétima iteração, fornecendo as seguintes estimativas:

$$
\begin{aligned}
& \hat{\sigma}_{p}^{2}=7035,84 \text { para } p=1,2, \ldots, 9 \\
& \hat{\lambda}=\mathrm{HF}=99,28 \\
& \hat{\sigma}_{e}^{2}=468,05
\end{aligned}
$$

Observa-se que as Tabelas 32, 33 e 34 apresentam as mesmas estimativas $\hat{\sigma}_{e}^{2}$, ou seja a estimativa do componente de variância residual não se alterou quando foram consideradas as estruturas de G iguais a CS, TOEP(1) e HF.

Utilizando-se essas estimativas tem-se que a matriz $G=H F$, conforme exemplificado na Tabela 2 , é dada por:

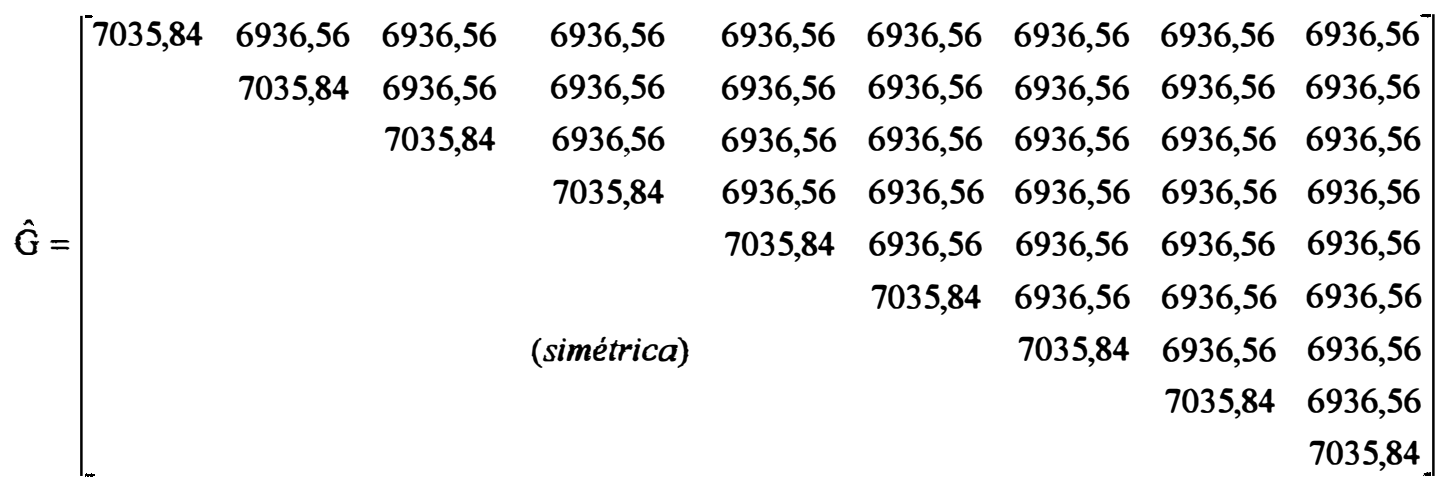

Observa-se, nesse caso, que $\hat{G}$ é do tipo uniforme e pode ser reescrita como:

$$
\hat{\mathrm{G}}=99,28 \mathbf{I}_{9}+6936,56 \mathbf{J}_{9}
$$

Assim, as estimativas apresentadas na Tabela 34 são:

$$
\begin{aligned}
& \hat{\mathrm{G}}=99,28 \mathbf{I}_{9}+6936,56 \mathbf{J}_{9} \\
& \hat{\sigma}_{e}^{2}=468,05
\end{aligned}
$$

Os valores dos critérios AIC e BIC, são, agora, menores do que aqueles observados nas Tabelas 31,32 e 33, ou seja

$$
\begin{aligned}
& \mathrm{AIC}=-93,9852-11=-104,985 \\
& \mathrm{BIC}=-93,9852-[11 \log (22-2)] / 2=-110,462
\end{aligned}
$$

indicando que a matriz HF é a "pior" dentre as quatro estudadas. 
$O$ teste para o efeito fixo, sexo, não se alterou como pode ser observado comparando-se com os resultados obtidos nas Tabelas 32 e 33 .

$\mathrm{Na}$ Tabela 34a tem-se os resultados dos critérios de informação AIC e BIC, utilizando-se o método REML e as estruturas de G, conforme visto em 4.2.5.

Tabela 34a. Resultados do AIC, do BIC e do nível nominal dos testes para o fator de efeitos fixos, sexo, pelo método REML de acordo com a estrutura de G adotada.

\begin{tabular}{ccccc}
\hline Estrutura de G & $\mathrm{q}^{(1)}$ & Valor do AIC & Valor do BIC & $\operatorname{Pr}>$ F (tipo III) \\
\hline VC & 3 & $-96,9807$ & $-98,4743$ & 0,0479 \\
CS & 3 & $-96,9852$ & $-98,4788$ & 0,0517 \\
TOEP(1) & 2 & $-95,9852$ & $-96,9810$ & 0,0517 \\
HF & 11 & $-104,985$ & $-110,462$ & 0,0517 \\
\hline
\end{tabular}

(1) número de parâmetros de covariâncias estimados no modelo.

Observando-se a Tabela 34a, verifica-se que a estrutura com o menor valor de AIC e BIC, considerada a "pior", é a estrutura de G = HF. Apesar disso, não há alteração no nível nominal do teste para o efeito fixo, sexo, ou seja o teste é significativo como nas demais estruturas.

Verifica-se, ainda, através dos valores AIC e BIC da Tabela 34a, que apenas a estrutura HF não é indicada para o conjunto modelo-dados, em questão. Dentre as mais indicadas há um ligeiro favoritismo para TOEP(1).

Assim, a escolha da estrutura de G mais apropriada, pelo método REML, para os dados da Tabela 3, não deve ser exclusivamente baseada nos critérios AIC e BIC. Na busca da estrutura mais adequada, deve-se também e principalmente considerar a natureza dos dados.

Com o objetivo de visualizar os valores dos critérios AIC e BIC fornecidos pelo PROC MIXED, segundo o método de estimação e a estrutura de G utilizada, construiuse a Tabela 35 .

Os critérios AIC e BIC são baseados na verossimilhança, e estão entre os mais comumente utilizados para selecionar a estrutura de covariâncias mais adequada aos dados (Bozdogan, 1987; Wolfinger, 1993). 
Tabela 35. Resultados dos critérios de informação de Akaike (AIC) e de Schwarz (BIC).

\begin{tabular}{cccc}
\hline Método & Estrutura de G & Valor do AIC & Valor do BIC \\
\hline \multirow{3}{*}{ MIVQUE0 } & VC & $-96,9962$ & $-98,4898$ \\
& CS & $-96,9889$ & $-98,4825$ \\
& TOEP(1) & $-95,9889$ & $-96,9847$ \\
HF & $-109,270$ & $-114,747$ \\
\hline \multirow{2}{*}{ ML } & VC & $-103,240$ & $-104,876$ \\
& CS & $(*)$ & $(*)$ \\
& TOEP(1) & $-102,290$ & $-103,381$ \\
& HF & $(*)$ & $(*)$ \\
\hline \multirow{2}{*}{ REML } & VC & $-96,9807$ & $-98,4743$ \\
& CS & $-96,9852$ & $-98,4788$ \\
& TOEP(1) & $-95,9852$ & $-96,9810$ \\
& HF & $-104,985$ & $-110,462$ \\
\hline
\end{tabular}

(*) o processo numérico para obter as estimativas ML não convergiu.

$\mathrm{Na}$ Tabela 35, comparando-se as estruturas de G: VC, CS, TOEP(1) e HF, observa-se que para os três métodos a "melhor" no sentido de maior valor AIC e BIC é a TOEP(1).

Conforme visto anteriormente, os critérios AIC e BIC não são adequados para comparar os métodos: MIVQUE0, ML e REML.

A menos que se conheça a matriz de covariâncias da população, torna-se pouco confortável para o usuário, afirmar qual é o melhor método. Uma comparação analítica torna-se viável para dados balanceados e mesmo assim deve ser feita com cautela, considerando-se cada modelo em particular. O método REML tem sido considerado (Searle, 1971; Corbeil \& Searle, 1976; Harville, 1977; Henderson, 1984; Searle et al., 1992, entre outros), o preferido para estimar componentes de variância de dados desbalanceados, conforme visto em 4.2.2.6. 
São apresentados na Tabela 36, os resultados dos testes de hipóteses para o fator de efeitos fixos, sexo, de acordo com as estruturas adotadas para a matriz $G$ e os métodos de estimação utilizados.

Tabela 36. Resultados dos testes do fator de efeitos fixos, sexo, usando PROC MIXED.

\begin{tabular}{cccc}
\hline Método & Estrutura de G & F Tipo III & $\operatorname{Pr}>$ F \\
\hline \multirow{3}{*}{ MIVQUE0 } & VC & 22,69 & 0,0414 \\
& CS & 17,33 & 0,0531 \\
& TOEP(1) & 17,33 & 0,0531 \\
& HF & 1,00 & 0,4229 \\
\hline \multirow{2}{*}{ ML } & VC & 30,35 & 0,0314 \\
& CS & $(*)$ & $(*)$ \\
& TOEP(1) & 23,76 & 0,0396 \\
& HF & $(*)$ & $(*)$ \\
\hline \multirow{2}{*}{ REML } & VC & 19,37 & 0,0479 \\
& CS & 17,87 & 0,0517 \\
& TOEP(1) & 17,87 & 0,0517 \\
& HF & 17,87 & 0,0517 \\
\hline
\end{tabular}

(*) o processo numérico para obter as estimativas ML não convergiu.

Como já visto em 4.2.4, os testes de hipóteses sobre os efeitos fixos dependem da estrutura da matriz $\mathrm{G}$ e do método de estimação utilizado.

$\mathrm{Na}$ Tabela 36, comparando-se os testes $\mathrm{F}$ para o fator de efeitos fixos, sexo, ao nivel de significância de 0,05 de acordo com as estruturas de G, para cada método separadamente tem-se:

- Para o MIVQUE0, o teste $F$ foi significativo para $G=V C$, bem próximo da significância para as estruturas, CS e TOEP(1), estas sendo iguais entre si, e afastandose sensivelmente da significância para a estrutura $\mathrm{HF}$. 
- Para o ML, o teste F foi significativo para as estruturas de G iguais a VC e TOEP(1), não sendo possível tal verificação para as estruturas CS e HF para as quais o processo numérico para obter as estimativas não convergiu.

- Para o REML, o teste $F$ foi significativo para a estrutura de $G=V C$, e bem próxima das significância para as demais estruturas. As demais estruturas apresentaram o mesmo nível nominal, inclusive a estrutura HF. Isto se deve, provavelmente, a própria filisofia do método REML que considera isoladamente a parte fixa e a parte aleatória do modelo, conforme discutido em 4.2.2.6.

Com o objetivo de ilustrar o desempenho dos procedimentos: MIXED e VARCOMP, são apresentados na Tabela 37 , as estimativas dos componentes de variância, resguardando-se as disponibilidades do PROC VARCOMP que utiliza, apenas a estrutura VC, e do PROC MIXED que utiliza os métodos MIVQUE0, ML e REML.

Tabela 37. Estimativas dos componentes de variância utilizando-se os procedimentos VARCOMP e MIXED.

\begin{tabular}{cccc}
\hline \multirow{2}{*}{ Método } & Parâmetro & \multicolumn{2}{c}{ Estimativas } \\
\cline { 3 - 4 } & & PROC VARCOMP & PROC MIXED \\
\hline \multirow{2}{*}{ MIVQUE0 } & $\hat{\sigma}_{\text {touro }}^{2}$ & 146,14 & 146,14 \\
& $\hat{\sigma}_{\text {sexo+touro }}^{2}$ & 49,16 & 49,16 \\
& $\hat{\sigma}_{e}^{2}$ & 474,15 & 474,15 \\
\hline \multirow{2}{*}{ ML } & $\hat{\sigma}_{\text {touro }}^{2}$ & 78,75 & 78,74 \\
& $\hat{\sigma}_{\text {sexo*touro }}^{2}$ & 7,54 & 7,54 \\
& $\hat{\sigma}_{e}^{2}$ & 471,16 & 471,18 \\
\hline \multirow{2}{*}{ REML } & $\hat{\sigma}_{\text {touro }}^{2}$ & 118,05 & 118,05 \\
& $\hat{\sigma}_{\text {sexorouro }}^{2}$ & 80,93 & 80,93 \\
& $\hat{\sigma}_{e}^{2}$ & 469,81 & 469,81 \\
\hline
\end{tabular}


Observa-se na Tabela 37 que as estimativas dos componentes de variância por ambos os procedimentos quando $\mathrm{G}=\mathrm{VC}$ são praticamente iguais.

Com o objetivo de ilustrar o desempenho dos procedimentos: MIXED e GLM, são apresentados na Tabela 38, o nível nominal para os testes dos tipos I e III para o fator de efeitos fixos, sexo, resguardando-se as disponibilidades do PROC GLM que utiliza, apenas a estrutura VC e o método ANOVA, e do PROC MIXED que utiliza os métodos MIVQUE0, ML e REML.

Tabela 38. Nível nominal dos testes dos tipos I e III para o fator de efeitos fixo, sexo, utilizando-se os procedimentos GLM e MIXED.

\begin{tabular}{cccc}
\hline \multirow{2}{*}{ Procedimento } & Método & \multicolumn{2}{c}{ Nivel nominal do teste $(\operatorname{Pr}>\mathrm{F})$} \\
\cline { 3 - 4 } & & Tipo I & Tipo III \\
\hline \multirow{2}{*}{ GLM } & ANOVA & 0,0514 & 0,0496 \\
\hline \multirow{2}{*}{ MIXED } & MIVQUE0 & 0,0414 & 0,0414 \\
& ML & 0,0314 & 0,0314 \\
& REML & 0,0479 & 0,0479 \\
\hline
\end{tabular}

Observa-se na Tabela 38 que os nível nominal para os testes do fator de efeitos fixos, sexo obtidos por ambos os procedimentos quando $G=V C$ são praticamente iguais. 


\section{CONCLUSÕES}

Face aos resultados obtidos, concluiu-se que:

\section{- Quanto ao Sistema Estatístico SAS}

O Sistema Estatístico SAS apresenta grande flexibilidade para o ajuste de modelos mistos através dos procedimentos abordados: GLM, VARCOMP e MIXED, destacando-se a excelente performance do procedimento MIXED.

O PROC GLM mesmo sendo um procedimento direcionado para modelos de efeitos fixos, forneceu, através da declaração RANDOM e da opção TEST, resultados plenamente compatíveis no tocante aos testes de hipóteses para o fator de efeitos fixos, sexo. Ressalta-se aqui a importância das esperanças dos quadrados médios, fornecidas nos quadros de análise de variância, que orientam o usuário na busca de testes apropriados. O PROC GLM, que utiliza apenas a estrutura VC, não forneceu as estimativas dos componentes de variância bem como as estimativas das matrizes de variâncias e covariâncias.

O PROC VARCOMP sendo direcionado para modelos de efeitos aleatórios, mostrou-se, através da opção FLXED da declaração MODEL, adequado para a parte aleatória do modelo misto. Forneceu estimativas dos componentes de variância por meio dos métodos: Tipo I ( método III de Henderson), MIVQUE0, ML e REML, apresentado como default o método MIVQUE0. Forneceu também, estimativas da matriz de variâncias e covariâncias assintóticas, para os métodos ML e REML. O PROC 
VARCOMP que, como o GLM, utiliza apenas a estrutura VC, não forneceu os testes de hipóteses para o fator de efeitos fixos, sexo.

O PROC MIXED é o procedimento do SAS apropriado para a análise de modelos mistos, pois permite uma especificação mais geral da matriz de covariâncias dos efeitos aleatórios $G$ e do resíduo, que neste trabalho foi tomada como $R=I \sigma^{2}$. Através da opção TYPE da declaração RANDOM, foi possível especificar a estrutura de G. Aqui foram consideradas as estruturas VC, CS, TOEP(1) e HF, sendo VC a estrutura default. O PROC MIXED forneceu as estimativas dos componentes de variância por meio dos métodos: MIVQUE0, ML e REML. Forneceu também, os critérios AIC e BIC, ambos baseados na verossimilhança, para comparar as estruturas utilizadas. Além disso, apresentou os testes de hipóteses dos tipos I e III para o fator de efeitos fixos, sexo.

\section{- Quanto as análises do conjunto de dados utilizado como exemplo}

Utilizando-se os critérios AIC e BIC, para comparar as estruturas adotadas, concluiu-se que apenas $G=\mathrm{HF}$ não foi indicada para descrever a estrutura de covariâncias dos dados. A "melhor" no sentido de maior valor AIC e BIC foi $\mathrm{G}=\mathrm{TOEP}(1)$, para os três métodos de estimação: MIVQUE0, ML e REML.

O nível nominal dos testes de hipóteses dos tipos I e III para o fator de efeitos fixos, sexo, foram semelhantes quando foi utilizada a estrutura $G=V C$ tanto no PROC GLM quanto no PROC MIXED. No tocante as demais estruturas utilizadas neste estudo, disponíveis apenas no PROC MIXED, apenas a estrutura $G=H F$ alterou sensivelmente o nível nominal em relação as demais.

Quanto as estimativas dos componentes de variância, uma comparação só foi possivel dentro da estrutura $G=V C$, que é comum ao PROC VARCOMP e ao PROC MIXED e nesse caso elas foram iguais "dentro" de cada um dos métodos MIVQUE0, ML e REML e diferentes "entre" os métodos. Em relação as demais estruturas utilizadas neste estudo e disponíveis no PROC MIXED, foi verificado um comportamento análogo, exceto para as estimativas dos componentes de variância obtidas pelos métodos MIVQUE0 e REML quando foram utilizadas as estruturas $G=C S$ e $G=\operatorname{TOEP}(1)$. 


\section{REFERÊNCIAS BIBLIOGRÁFICAS}

BARBIN, D. Componentes de variância. Piracicaba: ESALQ/USP, 1993. 108p.

BOZDOGAN, H. Model selection and Akaike's information criterion (AIC): The general theory and its analytical extensions. Psychometrika, v.52, n.3, p.345-370, 1987.

BROWN, K.G. Asymptotic behavior of MINQUE-type estimators of variance components. The Annals of Statistics, v.4, p.746-754, 1976.

CHRISTENSEN, R. Plane answers to complex questions: The theory of the linear model. New York: Springer-Verlag, 1987. 380p.

CIÓL, M.A. Métodos de estimação de componentes de variância em modelos mistos. Campinas, 1982. 126p. Dissertação (Mestrado) - Instituto de Matemática, Estatística e Ciência da Computação, Universidade Estadual de Campinas.

COCHRAN, W.G. Improvement by means of selection. In: BERKELEY SYMPOSIUM, 2., Los Angeles, 1951. Mathematical Statistical and Probability. Los Angeles: University of California Press, 1951. p.449-470.

CORBEIL, R. R.; SEARLE. S. R. Restricted maximum likelihood (REML) estimation of variance components in the mixed model. Technometrics, v. 18, p. 31-38, 1976. 
CUNNINGHAM, E.P.; HENDERSON, C.R. An iterative procedure for estimating fixed effects and variance components in mixed model situations. Biometrics, v.24 p.13$25,1968$.

FREITAS, A.R. Comparação de métodos para estimação de componentes de variância e parâmetros afins de múltiplos caracteres em bovinos. Piracicaba, 1991. 170p. Tese (Doutorado) - Escola Superior de Agricultura "Luiz de Queiroz", Universidade de São Paulo.

GIESBRECHT, F.G. An efficient procedure for computing MINQUE of variance components and generalized least squares estimates of fixed effects. Communications in Statistics - Part A: Theory and Methods, v.12, p.2169-2197, 1983.

GILMOUR, A.R.; THOMPSON, R.; CULLIS, B.R. Average information REML: an efficient algorithm for variance parameter estimation in linear mixed models. Biometrics, v.51, p.1440-1450, 1995.

HARTLEY, H.O.; RAO, J.N.K. Maximum likelihood estimation for the mixed analysis of variance model. Biometrika, v. 54, p.93-108, 1967.

HARVILLE, D.A. Maximum likelihood approaches to variances component estimation and to related problems. Journal of the American Statistical Association, v.72, p.320-340, 1977.

HEMMERLE, W.J.; HARTLEY, H.O. Computing maximum likelohood estimates for the mixed A.O.V. model using the W-transformation. Technometrics, v. 15, p.819$831,1973$.

HENDERSON, C.R. Estimation of variance and covariance components. Biometrics, v.9, p.226-252, 1953. 
HENDERSON, C.R. Sire evaluation and genetic trends. In: ANIMAL BREEDING AND GENETICS SYMPOSIUM, 10, Champaign, 1973. American Society of Animal Science. Champaign, 1973. p.10-41

HENDERSON, C.R. Applications of linear models in animal breeding. Ontario: University of Guelph, 1984. 462p.

HENDERSON, C.R.; KEMPTHORNE, O.; SEARLE, S.R.; VON KROSIGK, C.M. The estimation of environmental and genetic trends from records subject to culling. Biometrics, v.15, p.192-218, 1959.

HOCKING, R.R. The analysis of linear models. Monterey: Brooks/Cole Publishing Company, 1985. 385p.

HUYNH, H.; FELDT, L.S. Conditions under which mean square ratios in repeated measurements dsigns have exact F-distributions. Journal of the American Statistical Association, v.65, p.1582-1589, 1970.

IEMMA, A.F. Modelos lineares: uma introdução para profissionais da pesquisa agropecuária. Londrina: Imprensa Oficial do Estado do Paraná, 1987. 263p.

IEMMA, A.F. Testes de hipóteses em modelos lineares com amostras desequilibradas. Gembloux, 1991. 101p.

IEMMA, A.F. Análisis de varianza com datos desbalanceados. Colombia: Universidad Nacional da Colombia, 1993. 102p.

IEMMA, A.F. Análise de variância de dados desbalanceados. In: CONGRESSO BRASILEIRO DE USUÁRIOS DO SAS, 4., Piracicaba, 1995. Piracicaba: ESALQ/USP, 1995a. 111p. 
IEMMA, A.F. Que hipóteses estatísticas testamos através do "SAS" em presença de caselas vazias? Scientia Agricola, v.52, n.2, p.210-220, 1995b.

IEMMA, A.F. Hypothèses statistiques et fonctions estimables du logiciel SAS. Gembloux, 1997a. 30p.

IEMMA, A.F. Analyse des modeles mixtes par le logiciel SAS. Gembloux, 1997b. $31 \mathrm{p}$.

IEMMA, A.F.; PERRI, S.H.V. Procedimentos do sistema estatístico "SAS" para análise de modelos mistos. Multiciência, v.1, p.38-58, 1996.

IEMMA, A.F.; PERRI, S.H.V. Ajuste de modelos fixos desbalanceados através do sistema estatístico SAS. Piracicaba: ESALQ/USP, 1997. 99p.

KACKAR, R.N.; HARVILLE, D.A. Unbiasedness of two-stage estimation and prediction procedures ior mixed linear models. Communications in Statistics Part A. Theory and Methods, v.10, p.1249-1261, 1981.

KACKAR, R.N.; HARVILLE, D.A. Approximations for standard error of estimators of fixed and random effects in mixed linear models. Journal of the American Statistical Association, v.79, p.853-861, 1984.

LATOUR, D.; LATOUR, K.; WOLFINGER, R.D. Getting started with PROC MLXED. Cary: SAS Institute, 1994. 121p.

LITTELL, R.C.; MILLIKEN, G.A.; STROUP, W.W.; WOLFINGER, R.D. SAS system for mixed models. Cary: SAS Institute, 1996. 633p.

LOPES, P.S.; MARTINS, E.N.; SILVA, M.A.; REGAZZI, A.J. Estimação de componentes de variância. Viçosa: Universidade Federal de Viçosa, 1993. 61p. 
MILLER, J.J. Asymptotic properties of maximum likelihood estimates in the mixed model of the analysis of variance. The Annals of Statistics, v.5, p.746-762, 1977.

MILLIKEN, G.A.; JOHNSON, D.E. Analysis of messy data, volumel: designed experiments. New York: Chapman and Hall, 1984. 437p.

MONDARDO, M.; IEMMA, A.F. Sobre os quatro tipos de funções estimáveis fornecidas pelo PROC GLM do SAS para dados desbalanceados. Scientia Agricola, v. 55, n.2, p.172-182, 1998.

PATTERSON, H.D.; THOMPSON, R. Recovery of inter-block information when blocks sizes are unequal. Biometrika, v.58, p.545-554, 1971.

PERRI, S.H.V.; IEMMA, A.F. Ajuste de modelos mistos através do sistema estatístico SAS. Piracicaba: ESALQ/USP, 1996. 75p.

RAO, C.R. On the linear combination of observations and the general theory of least squares. Sankhyã, v.7, n.3, p.237-256, 1945.

RAO, C.R. Linear statistical inference and its applications. 1.ed. New York: John Wiley, 1965. 522p.

RAO, C.R. Estimation of heteroscedastic variances in linear models. Journal of the American Statistical Association, v.65, p.161-172, 1970.

RAO, C.R. Estimation of variance and covariance components - MINQUE Theory. Journal of Multivariate Analysis, v.1, p.257-275, 1971 a.

RAO, C.R. Minimum variance quadratic unbiased estimation of variance components. Journal of Multivariate Analysis, v. 1, p.445-456, 1971 b. 
RAO, C.R. Estimation of variance and covariance components in linear models. Journal of the American Statistical Association, v.67, p.112-115, 1972.

RICHARDSON, A.M.; WELSH, A.H. Robust restricted maximum likelihood in mixed linear models. Biometrics, v.51, p.1429-1439, 1995.

SAS INSTITUTE. SAS/STAT user's guide. Version 6, 4.ed., v.2. Cary, 1990. 846p.

SAS INSTITUTE. SAS/STAT software: changes and enhancements, release 6.07. Cary, 1992. chapter 16: The MIXED procedure. (SAS Technical Report P-229)

SAS INSTITUTE. Advanced general linear models with an emphasis on mixed models. Cary, 1996. 614p.

SAS INSTITUTE. SAS/STAT software: changes and enhancements through release 6.12. Cary, 1997. chapter 18: The MIXED procedure.

SCHEFFÉ, H. The analysis of variance. New York: John Wiley, 1959. 477p.

SCHWARZ, C.J. The mixed-model ANOVA: The truth, the computer packages, the books. Part 1: Balanced data. The American Statistician, v.47, n.1, p.48-59, 1993.

SEARLE, S.R. Another look at Henderson's methods of estimating variance components. Biometrics, v.24, p.749-778, 1968.

SEARLE, S.R. Large sample variances of maximum likelihood estimators of variance components using unbalanced data. Biometrics, v.26, p.505-524, 1970.

SEARLE, S.R. Linear models. New York: John Wiley, 1971. 532p. 
SEARLE, S.R. Prediction, mixed models and variance components. In: RELIABILITY AND BIOMETRY, Philadelphia, 1974. Society for Industrial and Applied Mathematics. Philadelphia: Proschan and Serfling, 1974. p.229-266.

SEARLE, S.R. Linear models for unbalanced data. New York: John Wiley, 1987. $536 \mathrm{p}$.

SEARLE, S.R. Best linear unbiased estimation in mixed models of the analysis of variance. In: ESSAYS IN THE HONOR OF FRANKLIN A. GRAYBILL, North Holland, 1988. Probability and Statistics. North Holland: J. N. Srivastava, Elsevier Science Publishers, 1988. p. 233- 241.

SEARLE, S.R.; CASELLA, G.; McCULLOCH, C. Variance components. New York: John Wiley, 1992. 501p.

SERAPHIN, J.C. Comparação numérica de três estimadores de componentes de variância em alguns modelos genético-estatísticos de cruzamentos. Piracicaba, 1984. 74p. Dissertação (Mestrado) - Escola Superior de Agricultura "Luiz de Queiroz", Universidade de São Paulo.

SMITH, D. W.; HOCKING, R. R. Maximum likelihood analysis of the mixed model: the balanced case. Communications in Statistics. Part A - Theory and Methods, v.7, p.1253-1266, 1978.

SWALLOW, W.H.; MONAHAN, J.F. Monte Carlo comparison of ANOVA, MINQUE, REML, and Ml estimators of variance components. Technometrics, v.26, p.47-57, 1984.

VALÉRIO FILHO, W.V. Métodos de Henderson para componentes da variância de dados não balanceados. Piracicaba, 1983. 137p. Dissertação (Mestrado) - Escola Superior de Agricultura "Luiz de Queiroz", Universidade de São Paulo. 
VALÉRIO FILHO, W.V. Comparação de métodos para estimação de componentes da variầncia através de simulação de dados. Piracicaba, 1991. 160p. Tese (Doutorado) Escola Superior de Agricultura "Luiz de Queiroz", Universidade de São Paulo.

VERNEQUE, R.S. Procedimentos numéricos e estimação de componentes de covariância em análise multivariada pelo método da máxima verossimilhança restrita modelos mistos aplicados ao melhoramento animal. Piracicaba, 1994. 57p. Tese (Doutorado) - Escola Superior de Agricultura "Luiz de Queiroz", Universidade de São Paulo.

WOLFINGER, R. Covariance structure selection in general mixed models. Communications in Statistics Simulation and Computation, v.22, n.4, p.1079$1106,1993$.

WOLFINGER, R.; TOBIAS, R.; SALL, J. Computing gaussian likelihoods and their derivatives for general linear mixed models. SIAM Journal on Scientific Computing, v.15, n.6, p.1294-1310, 1994. 